ESAIM: M2AN 46 (2012) 759-796

DOI: $10.1051 / \mathrm{m} 2 \mathrm{an} / 2011064$
ESAIM: Mathematical Modelling and Numerical Analysis

www.esaim-m2an.org

\title{
A MULTISCALE MORTAR MULTIPOINT FLUX MIXED FINITE ELEMENT METHOD
}

\author{
Mary Fanett Wheeler ${ }^{1}$, Guangri XuE ${ }^{2}$ And Ivan Yotov ${ }^{3}$
}

\begin{abstract}
In this paper, we develop a multiscale mortar multipoint flux mixed finite element method for second order elliptic problems. The equations in the coarse elements (or subdomains) are discretized on a fine grid scale by a multipoint flux mixed finite element method that reduces to cell-centered finite differences on irregular grids. The subdomain grids do not have to match across the interfaces. Continuity of flux between coarse elements is imposed via a mortar finite element space on a coarse grid scale. With an appropriate choice of polynomial degree of the mortar space, we derive optimal order convergence on the fine scale for both the multiscale pressure and velocity, as well as the coarse scale mortar pressure. Some superconvergence results are also derived. The algebraic system is reduced via a non-overlapping domain decomposition to a coarse scale mortar interface problem that is solved using a multiscale flux basis. Numerical experiments are presented to confirm the theory and illustrate the efficiency and flexibility of the method.
\end{abstract}

Mathematics Subject Classification. 65N06, 65N12, 65N15, 65N22, 65N30, 76S05.

Received August 4, 2010.

Published online February 3, 2012.

\section{INTRODUCTION}

We consider a second order linear elliptic equation written in a mixed form. Introducing a flux variable, we solve for a scalar function $p$ and a vector function $\mathbf{u}$ that satisfy

$$
\begin{array}{rlrl}
\mathbf{u} & =-K \nabla p & & \text { in } \Omega, \\
\nabla \cdot \mathbf{u} & =f & & \text { in } \Omega, \\
p=g & & \text { on } \partial \Omega,
\end{array}
$$

Keywords and phrases. Multiscale, mixed finite element, mortar finite element, multipoint flux approximation, cell-centered finite difference, full tensor coefficient, multiblock, nonmatching grids, quadrilaterals, hexahedra.

1 Center for Subsurface Modeling, Institute for Computational Engineering and Sciences, The University of Texas at Austin, Austin, 78712 TX, USA. mfw@ices.utexas.edu,

partially supported by the NSF-CDI under contract number DMS 0835745, the DOE grant DE-FGO2-04ER25617, and the Center for Frontiers of Subsurface Energy Security under Contract No. DE-SC0001114.

2 Center for Subsurface Modeling, Institute for Computational Engineering and Sciences, The University of Texas at Austin, Austin, 78712 TX, USA. gxue@ices.utexas.edu,

supported by Award No. KUS-F1-032-04, made by King Abdullah University of Science and Technology (KAUST).

3 Department of Mathematics, University of Pittsburgh, Pittsburgh, 15260 PA, USA. yotov@math.pitt.edu, partially supported by the DOE grant DE-FG02-04ER25618, the NSF grant DMS 0813901, and the J. Tinsley Oden Faculty Fellowship, ICES, The University of Texas at Austin. 
where $\Omega \subset \mathbb{R}^{d}, d=2,3$, is a polygonal domain with Lipschitz continuous boundary and $K$ is a symmetric, uniformly positive definite tensor with $L^{\infty}(\Omega)$ components, satisfying, for some $0<k_{0} \leq k_{1}<\infty$,

$$
k_{0} \xi^{T} \xi \leq \xi^{T} K(\mathbf{x}) \xi \leq k_{1} \xi^{T} \xi \quad \forall \mathbf{x} \in \Omega \forall \xi \in \mathbb{R}^{d} .
$$

We assume that $f \in L^{2}(\Omega)$ and $g \in H^{1 / 2}(\partial \Omega)$. The choice of Dirichlet boundary condition is made for simplicity. More general boundary conditions can also be treated. In porous media applications, the system (1.1)-(1.3) models single phase Darcy flow, where $p$ is the pressure, $\mathbf{u}$ is the velocity, and $K$ represents the rock permeability divided by fluid kinematic viscosity.

To alleviate the computational burden due to the solution dependence on a large range of scales, a multiscale mortar mixed finite element method for the the numerical approximation to (1.1)-(1.3) was developed in [12], using mortar mixed finite elements [11] and non-overlapping domain decomposition [34]. In this paper we develop a new multiscale mortar mixed method that uses the multipoint flux mixed finite element (MFMFE) $[39,56]$ for subdomain discretization.

The MFMFE method was motivated by the multipoint flux approximation (MPFA) method [3, 4, 6, 7, 27, 28]. The latter method was originally developed as a non-variational finite volume method. It is locally mass conservative, accurate for rough grids and coefficients, and reduces to a cell-centered system for the pressures. In that sense it combines the advantages of MFE and several MFE-related methods.

MFE methods $[19,49]$ are commonly used for flow in porous media, as they provide accurate and locally mass conservative velocities and handle well rough coefficients. However, the resulting algebraic system is of saddle point type and involves both the pressure and the velocity. Various modifications have been developed to alleviate this problem, including the hybrid MFE method $[13,19]$ that reduces to a symmetric positive definite face-centered pressure system, as well as more efficient cell-centered formulations $[9,10,15,50,55]$ based on numerical quadrature for the velocity mass matrix in the lowest order Raviart-Thomas $[46,48,52]\left(\mathrm{RT}_{0}\right)$ case. In terms of efficiency, the cell-centered methods are comparable to the finite volume methods [30]. All of the above mentioned cell-centered methods exhibit certain accuracy limitations, either in terms of grids or coefficients (full tensor or discontinuous). Two other family of methods that are closely related to the $\mathrm{RT}_{0} \mathrm{MFE}$ method and perform well for rough grids and coefficients are the control volume mixed finite element (CVMFE) method [23] and the mimetic finite difference (MFD) methods [38]. However, as in the case of MFE methods, both methods require solving algebraic saddle point problems in their standard form.

The MPFA method handles accurately very general grids and discontinuous full tensor coefficients and at the same time reduces to a positive definite cell-centered algebraic system for the pressure. The analysis of the MPFA method has been done by formulating it as a MFE method with a special quadrature, see $[39,42,56]$ for the symmetric version on $O\left(h^{2}\right)$-perturbations of parallelograms and parallelepipeds, respectively, as well as [41,57] for the non-symmetric version on general quadrilaterals and hexahedra, respectively. A non-symmetric MFD method on polyhedral elements that reduces to a cell-centered pressure system using a MPFA-type velocity elimination is developed and analyzed in [44]. Alternative approaches relating finite volume methods to mixed finite element methods on simplicial meshes without using numerical quadrature have been developed in $[53,58]$.

Here we use for subdomain discretizations the MFMFE method developed in [56] for simplicial elements and quadrilaterals and extended in [39] to hexahedra, see also closely related method on simplicial elements [22]. Since the MPFA method uses sub-edge or sub-face fluxes to allow for local flux elimination, the MFMFE method is based on the lowest order Brezzi-Douglas-Marini space, $\mathrm{BDM}_{1}[20]$, and its extension to hexahedra, $\mathrm{BDDF}_{1}[21,39]$, which have similar velocity degrees of freedom. The $\mathrm{BDDF}_{1}$ space was enhanced in [39] with six additional curl basis functions. The resulting space has bilinear normal traces on the faces, thus four degrees of freedom per face, which allows for a MPFA-type local velocity elimination. This is done via a special quadrature rule for the velocity mass matrix that reduces it to a block-diagonal form, with blocks corresponding to the mesh vertices. As a result, the velocity can be easily eliminated, leading to a cell-centered system for the pressure.

The permeability $K$ in (1.2) is usually highly heterogeneous and varies on a multiplicity of scales. Resolving the solution on the finest scale is often computationally infeasible, necessitating multiscale approximations. In this paper we develop a new multiscale mortar MFMFE method. The approach is similar to the one in [12] for the 
multiscale mortar mixed finite element method. The latter was developed as an alternative to existing multiscale methods, such as the variational multiscale method $[8,37]$ and multiscale finite elements $[1,2,24,36,40]$. In the multiscale mortar approach, the domain is decomposed into a series of small subdomains (coarse grid) and the solution is resolved globally on the coarse grid and locally (on each coarse element) on a fine grid. We allow for geometrically nonconforming domain decompositions and non-matching grids across the interfaces. Continuity of flux is imposed weakly using a low degree-of-freedom mortar space defined on a coarse scale mortar grid. Mortar methods were first introduced in [16] for Galerkin finite elements. In this paper we consider the MFMFE method for subdomain discretizations. In this context, special care needs to be taken in imposing weak flux continuity through the mortar space. In particular, in the case of quadrilaterals and hexahedra, the method requires that the jump of the $\mathrm{RT}_{0}$-projections of the $\mathrm{BDM}_{1}$ or enhanced $\mathrm{BDDF}_{1}$ fluxes be orthogonal to the mortar space. On simplicial elements, the $\mathrm{RT}_{0}$-projection step is omitted. This condition is needed to ensure stability and accuracy of the method.

We solve the algebraic system resulting from the multiscale mortar MFMFE method via a non-overlapping domain decomposition algorithm $[11,12,34]$. By eliminating the subdomain unknowns, the global multiscale problem is reduced to a coarse interface problem, which is solved using an iterative method. Employing the approach from [32], we precompute a multiscale flux basis by solving in each subdomain Dirichlet fine scale local problems for each mortar degree of freedom associated with that subdomain. The subdomain problems are easy to solve due to their relatively small size. Furthermore, this is done in parallel without any interprocessor communication. Computing the action of the interface operator on every interface iteration is then reduced to a linear combination of the multiscale flux basis. The resulting method combines the efficiency of the multiscale mortar methodology with the accuracy and flexibility of the MFMFE subdomain discretizations.

We present a priori error analysis of the multiscale mortar MFMFE approximation. While the method is developed for general quadrilaterals and hexahedra, the analysis is restricted to $O\left(h^{2}\right)$-perturbations of parallelograms and parallelepipeds, see Section 5.1 for rigorous definitions. This restriction is typical for symmetric MFMFE formulations, see $[39,56]$. It is possible to extend the multiscale mortar methodology to the non-symmetric MFMFE method developed in $[41,57]$, thus relaxing the constraints on the element geometry. However, this is beyond the scope of this paper.

By using a higher order mortar approximation, we are able to compensate for the coarseness of the grid scale and maintain good (fine scale) overall accuracy. In particular, let $m$ be the degree of the mortar approximation polynomial, let $h$ be the size of the fine scale subdomain grids, and let $H$ be the size of coarse mortar grid on the interface. We show that the the velocity and pressure errors are $\mathcal{O}\left(H^{m+1 / 2}+h\right)$. On certain elements at certain discrete points we also show $\mathcal{O}\left(H^{m+1 / 2}+h H^{1 / 2}\right)$ velocity superconvergence and $\mathcal{O}\left(H^{m+3 / 2}+h H\right)$ pressure superconvergence. The analysis follows the general multiscale mortar framework developed in [12]. However, the different subdomain discretizations considered here lead to significant technical differences, requiring many arguments to be carried out in detail. These include both the construction and analysis of a weakly continuous projection operator in Section 5 and the error analysis in Sections 6 and 7. In the process we have also been able to reduce the global velocity regularity required for convergence to $\|\mathbf{u}\|_{r+1 / 2}, r>0$.

The paper is organized as follows. In Section 2 we present the variational formulation. The subdomain MFMFE discretization is described in Section 3. In Section 4 we introduce the multiscale mortar MFMFE method. Various approximation properties are presented in Section 5. The error analysis is developed in Section 6. A non-overlapping domain decomposition algorithm for solving the multiscale algebraic system is given in Section 7. The error in the mortar pressure is also analyzed in that section. The paper ends with a series of numerical experiments in Section 8.

Throughout this paper, we use for simplicity $X \lesssim(\gtrsim) Y$ to denote that there exists a constant $C$, independent of mesh sizes $h$ and $H$, such that $X \leq(\geq) C Y$. The notation $X \approx Y$ means that both $X \lesssim Y$ and $X \gtrsim Y$ hold.

For a domain $G \subset \mathbb{R}^{d}$, the $L^{2}(G)$ inner product and norm for scalar and vector valued functions are denoted $(\cdot, \cdot)_{G}$ and $\|\cdot\|_{G}$, respectively. The norms and seminorms of the Sobolev spaces $W^{k, p}(G), k \in \mathbb{R}, p>0$ are denoted by $\|\cdot\|_{k, p, G}$ and $|\cdot|_{k, p, G}$, respectively. The norms and seminorms of the Hilbert spaces $H^{k}(G)$ are denoted by $\|\cdot\|_{k, G}$ and $|\cdot|_{k, G}$, respectively. We omit $G$ in the subscript if $G=\Omega$. For a section of the domain, 
subdomain, or element boundary $S \subset \mathbb{R}^{d-1}$ we write $\langle\cdot, \cdot\rangle_{S}$ and $\|\cdot\|_{S}$ for the $L^{2}(S)$ inner product (or duality pairing) and norm, respectively. For a tensor-valued function $M$, let $\|M\|_{\alpha, \infty}=\max _{i, j}\left\|M_{i j}\right\|_{\alpha, \infty}$. Furthermore, let

$$
H(\operatorname{div} ; G)=\left\{\mathbf{v} \in\left(L^{2}(G)\right)^{d}: \nabla \cdot \mathbf{v} \in L^{2}(G)\right\}, \quad\|\mathbf{v}\|_{H(\operatorname{div} ; G)}=\left(\|\mathbf{v}\|_{G}^{2}+\|\nabla \cdot \mathbf{v}\|_{G}^{2}\right)^{1 / 2}
$$

\section{Multidomain variational Formulation}

A weak formulation of (1.1)-(1.3) can be written as: find $\mathbf{u} \in H(\operatorname{div} ; \Omega)$ and $p \in L^{2}(\Omega)$, such that

$$
\begin{aligned}
\left(K^{-1} \mathbf{u}, \mathbf{v}\right)-(p, \nabla \cdot \mathbf{v}) & =-\langle g, \mathbf{v} \cdot \mathbf{n}\rangle_{\partial \Omega}, & & \forall \mathbf{v} \in H(\operatorname{div} ; \Omega), \\
(\nabla \cdot \mathbf{u}, w) & =(f, w), & & \forall w \in L^{2}(\Omega) .
\end{aligned}
$$

It is well known $[19,49]$ that $(2.1)-(2.2)$ has a unique solution. Let $\Omega$ be decomposed into non-overlapping polygonal subdomains (blocks) $\Omega_{i}$, such that

$$
\bar{\Omega}=\bigcup_{i=1}^{n} \bar{\Omega}_{i} \text { and } \Omega_{i} \cap \Omega_{j} \neq \emptyset .
$$

Let $\Gamma_{i, j}=\partial \Omega_{i} \cap \partial \Omega_{j}$ denote the subdomain interfaces,

$$
\Gamma=\bigcup_{1 \leq i<j \leq n} \Gamma_{i, j}, \quad \text { and } \quad \Gamma_{i}=\partial \Omega_{i} \cap \Gamma=\partial \Omega_{i} \backslash \partial \Omega .
$$

The interfaces are assumed to be flat. We allow for non-conforming decompositions, i.e., interfaces may be subsets of subdomain faces. The multidomain formulation of (2.1)-(2.2) is based on the spaces

$$
\begin{array}{ll}
\mathbf{V}_{i}=H\left(\operatorname{div} ; \Omega_{i}\right), & \mathbf{V}=\bigoplus_{i=1}^{n} \mathbf{V}_{i}, \\
W_{i}=L^{2}\left(\Omega_{i}\right), & W=\bigoplus_{i=1}^{n} W_{i}=L^{2}(\Omega) .
\end{array}
$$

If the solution $(\mathbf{u}, p)$ of $(2.1)-(2.2)$ belongs to $H(\operatorname{div} ; \Omega) \times H^{1}(\Omega)$, it is well-known [19] that it satisfies, for $1 \leq i \leq n$,

$$
\begin{aligned}
\left(K^{-1} \mathbf{u}, \mathbf{v}\right)_{\Omega_{i}}- & (p, \nabla \cdot \mathbf{v})_{\Omega_{i}}=-\left\langle p, \mathbf{v} \cdot \mathbf{n}_{i}\right\rangle_{\Gamma_{i}}-\left\langle g, \mathbf{v} \cdot \mathbf{n}_{i}\right\rangle_{\partial \Omega_{i} \backslash \Gamma}, & & \forall \mathbf{v} \in \mathbf{V}_{i} \\
(\nabla \cdot \mathbf{u}, w)_{\Omega_{i}} & =(f, w)_{\Omega_{i}}, & & \forall w \in W_{i}
\end{aligned}
$$

where $\mathbf{n}_{i}$ is the outer unit normal vector to $\partial \Omega_{i}$.

\section{A multipoint flux mixed finite element method on Subdomains}

In this section we discuss the multipoint flux mixed finite element method used for subdomain discretizations. It is based on the lowest order $\mathrm{BDM}_{1}$ or $\mathrm{BDDF}_{1}$ elements with a quadrature rule, which allows for local velocity elimination and reduction to a cell-centered scheme for the pressure. The method is presented for simplices and general quadrilaterals and hexahedra. In Section 5.1, a further restriction on the geometry of non-affine elements is imposed for the convergence analysis. 

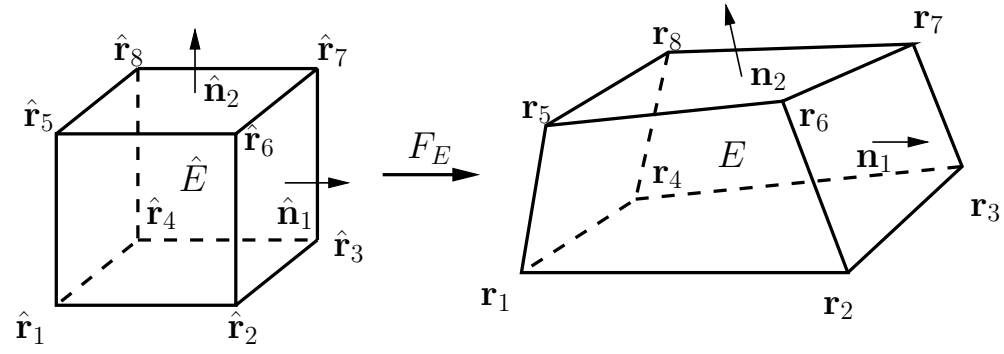

Figure 1. Trilinear hexahedral mapping.

\subsection{Finite element mappings}

Let $\mathcal{T}_{h, i}$ be a conforming, shape-regular, quasi-uniform partition of $\Omega_{i}, 1 \leq i \leq n$ [25]. The elements considered are two and three dimensional simplexes, convex quadrilaterals in two dimensions, and hexahedra in three dimensions. The hexahedra can have non-planar faces. For any element $E \in \mathcal{T}_{h, i}$, there exists a bijection mapping $F_{E}: \hat{E} \rightarrow E$, where $\hat{E}$ is a reference element. Denote the Jacobian matrix by $D F_{E}$ and let $J_{E}=\operatorname{det}\left(D F_{E}\right)$ where we assume that $\operatorname{sign}\left(J_{E}\right)>0$. Denote the inverse mapping by $F_{E}^{-1}$, its Jacobian matrix by $D F_{E}^{-1}$, and let $J_{F_{E}^{-1}}=\operatorname{det}\left(D F_{E}^{-1}\right)$. We have that

$$
D F_{E}^{-1}(x)=\left(D F_{E}\right)^{-1}(\hat{x}), \quad J_{F_{E}^{-1}}(x)=\frac{1}{J_{E}(\hat{x})} .
$$

In the case of convex hexahedra, $\hat{E}$ is the unit cube with vertices $\hat{\mathbf{r}}_{1}=(0,0,0)^{T}, \hat{\mathbf{r}}_{2}=(1,0,0)^{T}, \hat{\mathbf{r}}_{3}=(1,1,0)^{T}$, $\hat{\mathbf{r}}_{4}=(0,1,0)^{T}, \hat{\mathbf{r}}_{5}=(0,0,1)^{T}, \hat{\mathbf{r}}_{6}=(1,0,1)^{T}, \hat{\mathbf{r}}_{7}=(1,1,1)^{T}$, and $\hat{\mathbf{r}}_{8}=(0,1,1)^{T}$. Denote by $\mathbf{r}_{i}=\left(x_{i}, y_{i}, z_{i}\right)^{T}, i=$ $1, \ldots, 8$, the eight corresponding vertices of element $E$ as shown in Figure 1 . We note that the element can have non-planar faces. The outward unit normal vectors to the faces of $E$ and $\hat{E}$ are denoted by $\mathbf{n}_{i}$ and $\hat{\mathbf{n}}_{i}, i=1, \ldots, 6$, respectively. In this case $F_{E}$ is a trilinear mapping given by

$$
\begin{aligned}
F_{E}(\hat{\mathbf{r}})= & \mathbf{r}_{1}(1-\hat{x})(1-\hat{y})(1-\hat{z})+\mathbf{r}_{2} \hat{x}(1-\hat{y})(1-\hat{z})+\mathbf{r}_{3} \hat{x} \hat{y}(1-\hat{z})+\mathbf{r}_{4}(1-\hat{x}) \hat{y}(1-\hat{z}) \\
& +\mathbf{r}_{5}(1-\hat{x})(1-\hat{y}) \hat{z}+\mathbf{r}_{6} \hat{x}(1-\hat{y}) \hat{z}+\mathbf{r}_{7} \hat{x} \hat{y} \hat{z}+\mathbf{r}_{8}(1-\hat{x}) \hat{y} \hat{z} \\
= & \mathbf{r}_{1}+\mathbf{r}_{21} \hat{x}+\mathbf{r}_{41} \hat{y}+\mathbf{r}_{51} \hat{z}+\left(\mathbf{r}_{34}-\mathbf{r}_{21}\right) \hat{x} \hat{y}+\left(\mathbf{r}_{65}-\mathbf{r}_{21}\right) \hat{x} \hat{z}+\left(\mathbf{r}_{85}-\mathbf{r}_{41}\right) \hat{y} \hat{z} \\
& +\left(\mathbf{r}_{21}-\mathbf{r}_{34}-\mathbf{r}_{65}+\mathbf{r}_{78}\right) \hat{x} \hat{y} \hat{z},
\end{aligned}
$$

where $\mathbf{r}_{i j}=\mathbf{r}_{i}-\mathbf{r}_{j}$. It is easy to see that each component of $D F_{E}$ is a bilinear function of two space variables:

$$
\begin{aligned}
D F_{E}(\hat{\mathbf{r}})= & {\left[\mathbf{r}_{21}+\left(\mathbf{r}_{34}-\mathbf{r}_{21}\right) \hat{y}+\left(\mathbf{r}_{65}-\mathbf{r}_{21}\right) \hat{z}+\left(\mathbf{r}_{21}-\mathbf{r}_{34}-\mathbf{r}_{65}+\mathbf{r}_{78}\right) \hat{y} \hat{z},\right.} \\
& \mathbf{r}_{41}+\left(\mathbf{r}_{34}-\mathbf{r}_{21}\right) \hat{x}+\left(\mathbf{r}_{85}-\mathbf{r}_{41}\right) \hat{z}+\left(\mathbf{r}_{21}-\mathbf{r}_{34}-\mathbf{r}_{65}+\mathbf{r}_{78}\right) \hat{x} \hat{z}, \\
& \left.\mathbf{r}_{51}+\left(\mathbf{r}_{65}-\mathbf{r}_{21}\right) \hat{x}+\left(\mathbf{r}_{85}-\mathbf{r}_{41}\right) \hat{y}+\left(\mathbf{r}_{21}-\mathbf{r}_{34}-\mathbf{r}_{65}+\mathbf{r}_{78}\right) \hat{x} \hat{y}\right]
\end{aligned}
$$

In the case of tetrahedra, $\hat{E}$ is the reference tetrahedron with vertices $\hat{\mathbf{r}}_{1}=(0,0,0)^{T}, \hat{\mathbf{r}}_{2}=(1,0,0)^{T}, \hat{\mathbf{r}}_{3}=$ $(0,1,0)^{T}$, and $\hat{\mathbf{r}}_{4}=(0,0,1)^{T}$. Let $\mathbf{r}_{i}, i=1, \ldots, 4$, be the corresponding vertices of $E$. The linear mapping for tetrahedra has the form

$$
F_{E}(\hat{\mathbf{r}})=\mathbf{r}_{1}(1-\hat{x}-\hat{y}-\hat{z})+\mathbf{r}_{2} \hat{x}+\mathbf{r}_{3} \hat{y}+\mathbf{r}_{4} \hat{z}
$$

with respective Jacobian matrix and its determinant

$$
D F_{E}=\left[\mathbf{r}_{21}, \mathbf{r}_{31}, \mathbf{r}_{41}\right] \text { and } J_{E}=2|E|,
$$

where $|E|$ is the area of element $E$. 
The mappings in the cases of quadrilaterals and triangles are described similarly to the cases of hexahedra and tetrahedra, respectively. Note that in the case of simplicial elements the mapping is affine and the Jacobian matrix and its determinant are constants. This is not the case for quadrilaterals and hexahedra.

Using the above mapping definitions and the classical formula $\nabla \phi=D F_{E}^{-T} \hat{\nabla} \hat{\phi}$, for $\phi(\mathbf{r})=\hat{\phi}(\hat{\mathbf{r}})$, it is easy to see that for any face or edge $e_{i} \subset E$,

$$
\mathbf{n}_{i}=\frac{D F_{E}^{-T} \hat{\mathbf{n}}_{i}}{\left|D F_{E}^{-T} \hat{\mathbf{n}}_{i}\right|} .
$$

Also, the shape regularity and quasi-uniformity of the grids imply that for all elements $E \in \mathcal{T}_{h}$,

$$
\left\|D F_{E}\right\|_{0, \infty, \hat{E}} \lesssim h, \quad\left\|J_{E}\right\|_{0, \infty, \hat{E}} \bar{\sim} h^{d}, \quad\left\|D F_{E}^{-1}\right\|_{0, \infty, E} \lesssim h^{-1}, \quad\left\|J_{F_{E}^{-1}}\right\|_{0, \infty, \hat{E}} \bar{\sim} h^{-d} .
$$

\subsection{Mixed finite element spaces}

We introduce four finite element spaces with respect to the four types of elements considered in this paper. Let $\hat{\mathbf{V}}(\hat{E})$ and $\hat{W}(\hat{E})$ denote the finite element spaces on the reference element $\hat{E}$.

For simplicial elements, we employ $\mathrm{BDM}_{1}[20]$ on triangles and $\mathrm{BDDF}_{1}[21]$ on tetrahedra:

$$
\hat{\mathbf{V}}(\hat{E})=\left(P_{1}(\hat{E})\right)^{d}, \quad \hat{W}(\hat{E})=P_{0}(\hat{E}),
$$

where $P_{k}$ denotes the space of polynomials of degree $\leq k$.

On the unit square, we employ $\mathrm{BDM}_{1}[20]$ :

$$
\hat{\mathbf{V}}(\hat{E})=\left(P_{1}(\hat{E})\right)^{2}+r \operatorname{curl}\left(\hat{x}^{2} \hat{y}\right)+s \operatorname{curl}\left(\hat{x} \hat{y}^{2}\right), \quad \hat{W}(\hat{E})=P_{0}(\hat{E}),
$$

where $r$ and $s$ are real constants.

On the unit cube, we employ the enhanced $\mathrm{BDDF}_{1}$ space [39]:

$$
\begin{aligned}
\hat{\mathbf{V}}(\hat{E})= & \operatorname{BDDF}_{1}(\hat{E})+r_{2} \operatorname{curl}\left(0,0, \hat{x}^{2} \hat{z}\right)^{T}+r_{3} \operatorname{curl}\left(0,0, \hat{x} \hat{y}^{2} \hat{z}\right)^{T}+s_{2} \operatorname{curl}\left(\hat{x} \hat{y}^{2}, 0,0\right)^{T} \\
& +s_{3} \operatorname{curl}\left(\hat{x} \hat{y}^{2} \hat{z}, 0,0\right)^{T}+t_{2} \operatorname{curl}\left(0, \hat{y} \hat{z}^{2}, 0\right)^{T}+t_{3} \operatorname{curl}\left(0, \hat{x} \hat{y} \hat{z}^{2}, 0\right)^{T}, \\
\hat{W}(\hat{E})= & P_{0}(\hat{E}),
\end{aligned}
$$

where the $\mathrm{BDDF}_{1}$ space on unit cube [21] is defined as

$$
\begin{aligned}
\operatorname{BDDF}_{1}(\hat{E})= & \left(P_{1}(\hat{E})\right)^{3}+r_{0} \operatorname{curl}(0,0, \hat{x} \hat{y} \hat{z})^{T}+r_{1} \operatorname{curl}\left(0,0, \hat{x} \hat{y}^{2}\right)^{T}+s_{0} \operatorname{curl}(\hat{x} \hat{y} \hat{z}, 0,0)^{T} \\
& +s_{1} \operatorname{curl}\left(\hat{y} \hat{z}^{2}, 0,0\right)^{T}+t_{0} \operatorname{curl}(0, \hat{x} \hat{y} \hat{z}, 0)^{T}+t_{1} \operatorname{curl}\left(0, \hat{x}^{2} \hat{z}, 0\right)^{T},
\end{aligned}
$$

where $r_{i}, s_{i}, t_{i}, i=0, \ldots 3$, are real constants.

Note that in all four cases

$$
\hat{\nabla} \cdot \hat{\mathbf{V}}(\hat{E})=\hat{W}(\hat{E}) .
$$

On any face (edge in 2D) $\hat{e} \in \hat{E}$, for all $\hat{\mathbf{v}} \in \hat{\mathbf{V}}(\hat{E}), \hat{\mathbf{v}} \cdot \hat{\mathbf{n}}_{\hat{e}} \in P_{1}(\hat{e})$ on the reference square or simplex, and $\hat{\mathbf{v}} \cdot \hat{\mathbf{n}}_{\hat{e}} \in Q_{1}(\hat{e})$ on the reference cube, where $Q_{1}(\hat{e})$ is the space of bilinear functions on $\hat{e}$.

The degrees of freedom for $\hat{\mathbf{V}}(\hat{E})$ are chosen to be the values of $\hat{\mathbf{v}} \cdot \hat{\mathbf{n}}_{\hat{e}}$ at the vertices of $\hat{e}$, for each face (edge) $\hat{e}$. This choice gives certain orthogonalities for the quadrature rule introduced in the next section and leads to a cell-centered pressure scheme.

The spaces $\mathbf{V}(E)$ and $W(E)$ on any physical element $E \in \mathcal{T}_{h}$ are defined, respectively, via the Piola transformation

$$
\mathbf{v} \leftrightarrow \hat{\mathbf{v}}: \mathbf{v}=\frac{1}{J_{E}} D F_{E} \hat{\mathbf{v}} \circ F_{E}^{-1}
$$

and standard scalar transformation

$$
w \leftrightarrow \hat{w}: w=\hat{w} \circ F_{E}^{-1}
$$


Under these transformations, the divergence and the normal components of the velocity vectors on the faces (edges) are preserved [19]:

$$
(\nabla \cdot \mathbf{v}, w)_{E}=(\hat{\nabla} \cdot \hat{\mathbf{v}}, \hat{w})_{\hat{E}} \quad \text { and } \quad\left\langle\mathbf{v} \cdot \mathbf{n}_{e}, w\right\rangle_{e}=\left\langle\hat{\mathbf{v}} \cdot \hat{\mathbf{n}}_{\hat{e}}, \hat{w}\right\rangle_{\hat{e}} .
$$

In addition, (3.5) implies that

$$
\mathbf{v} \cdot \mathbf{n}_{e}=\frac{1}{\left|J_{E} D F_{E}^{-T} \hat{\mathbf{n}}_{\hat{e}}\right|} \hat{\mathbf{v}} \cdot \hat{\mathbf{n}}_{\hat{e}}
$$

and (3.11) implies that

$$
\nabla \cdot \mathbf{v}=\left(\frac{1}{J_{E}} \hat{\nabla} \cdot \hat{\mathbf{v}}\right) \circ F_{E}^{-1}(\mathbf{x})
$$

On quadrilaterals or hexahedra, $\nabla \cdot \mathbf{v} \neq$ constant since $J_{E}$ is not constant.

The finite element spaces $\mathbf{V}_{h, i}$ and $W_{h, i}$ on subdomain $\Omega_{i}$ are given by

$$
\begin{aligned}
& \mathbf{V}_{h, i}=\left\{\mathbf{v} \in \mathbf{V}:\left.\quad \mathbf{v}\right|_{E} \leftrightarrow \hat{\mathbf{v}}, \hat{\mathbf{v}} \in \hat{\mathbf{V}}(\hat{E}), \quad \forall E \in \mathcal{T}_{h, i}\right\}, \\
& W_{h, i}=\left\{w \in W:\left.\quad w\right|_{E} \leftrightarrow \hat{w}, \hat{w} \in \hat{W}(\hat{E}), \quad \forall E \in \mathcal{T}_{h, i}\right\} .
\end{aligned}
$$

The global mixed finite element spaces are defined as

$$
\mathbf{V}_{h}=\bigoplus_{i=1}^{n} \mathbf{V}_{h, i}, \quad W_{h}=\bigoplus_{i=1}^{n} W_{h, i} .
$$

We recall the projection operator in the space $\mathbf{V}_{h, i}$. The operator $\hat{\Pi}:\left(H^{1}(\hat{E})\right)^{d} \rightarrow \hat{\mathbf{V}}(\hat{E})$ is defined locally on each element by

$$
\left\langle(\hat{\Pi} \hat{\mathbf{q}}-\hat{\mathbf{q}}) \cdot \hat{\mathbf{n}}_{\hat{e}}, \hat{q}_{1}\right\rangle_{\hat{e}}=0, \quad \forall \hat{e} \subset \partial \hat{E},
$$

where $\hat{q}_{1} \in P_{1}(\hat{e})$ when $\hat{E}$ is the unit square or simplicial element, and $\hat{q}_{1} \in Q_{1}(\hat{e})$ when $\hat{E}$ is the unit cube. The global operator $\Pi: \mathbf{V} \cap\left(H^{1}(\Omega)\right)^{d} \rightarrow \mathbf{V}_{h}$ on each element $E$ is defined via the Piola transformation:

$$
\Pi \mathbf{q} \leftrightarrow \widehat{\Pi \mathbf{q}}, \quad \widehat{\Pi \mathbf{q}}=\hat{\Pi} \hat{\mathbf{q}} .
$$

Furthermore, (3.11), (3.15), and (3.16) imply that $\Pi \mathbf{q} \cdot \mathbf{n}$ is continuous across element interfaces, which gives $\Pi \mathbf{q} \in \mathbf{V}_{h, i}$, and that

$$
(\nabla \cdot(\Pi \mathbf{q}-\mathbf{q}), w)_{\Omega_{i}}=0, \quad \forall w \in W_{h, i}
$$

In the analysis, we also need similar projection operators onto the lowest order Raviart-Thomas $[46,48]$ spaces. The $R T_{0}$ spaces are defined on the reference cube and the reference tetrahedron, respectively, as

$$
\hat{\mathbf{V}}^{R T}(\hat{E})=\left(\begin{array}{c}
r_{1}+s_{1} \hat{x} \\
r_{2}+s_{2} \hat{y} \\
r_{3}+s_{3} \hat{z}
\end{array}\right), \quad \hat{W}^{R T}(\hat{E})=P_{0}(\hat{E}),
$$

and

$$
\hat{\mathbf{V}}^{R T}(\hat{E})=\left(\begin{array}{c}
r_{1}+s \hat{x} \\
r_{2}+s \hat{y} \\
r_{3}+s \hat{z}
\end{array}\right), \quad \hat{W}^{R T}(\hat{E})=P_{0}(\hat{E})
$$

with similar definitions in two dimensions, where $s, r_{i}, s_{i}(i=1,2,3)$ are constants.

In all cases $\hat{\nabla} \cdot \hat{\mathbf{V}}^{R T}=\hat{W}^{R T}(\hat{E})$ and $\hat{\mathbf{v}} \cdot \hat{\mathbf{n}}_{e} \in P_{0}(\hat{e})$. The degrees of freedom of $\hat{\mathbf{V}}^{R T}(\hat{E})$ are chosen to be the values of $\hat{\mathbf{v}} \cdot \hat{\mathbf{n}}_{\hat{e}}$ at the midpoints of all faces (edges) of $\hat{E}$. The projection operator $\hat{\Pi}^{R T}:\left(H^{1}(\hat{E})\right)^{d} \rightarrow \hat{\mathbf{V}}^{R T}(\hat{E})$ satisfies

$$
\left\langle\left(\hat{\Pi}^{R T} \hat{\mathbf{q}}-\hat{\mathbf{q}}\right) \cdot \hat{\mathbf{n}}_{\hat{e}}, \hat{q}_{0}\right\rangle_{\hat{e}}=0, \quad \forall \hat{e} \subset \partial \hat{E}, \quad \forall q_{0} \in P_{0}(\hat{E}) .
$$


The spaces $\mathbf{V}_{h}^{R T}$ and $W_{h}^{R T}$ on $\Omega$ and the projection operator $\Pi^{R T}:\left(H^{1}(\Omega)\right)^{d} \rightarrow \mathbf{V}_{h}^{R T}$ are defined similarly to the case of $\mathbf{V}_{h}$ and $W_{h}$. By definition, we have

$$
\mathbf{V}_{h, i}^{R T} \subset \mathbf{V}_{h, i} \quad W_{h, i}^{R T}=W_{h, i} .
$$

The projection operator $\Pi^{R T}$ satisfies

$$
\begin{gathered}
\left(\nabla \cdot\left(\Pi^{R T} \mathbf{q}-\mathbf{q}\right), w\right)=0, \quad \forall w \in W_{h, i}^{R T}, \\
\nabla \cdot \Pi^{R T} \mathbf{v}=\nabla \cdot \mathbf{v}, \quad \forall \mathbf{v} \in \mathbf{V}_{h, i},
\end{gathered}
$$

and for all element $E \in \mathcal{T}_{h, i}$,

$$
\left\|\Pi^{R T} \mathbf{v}\right\|_{E} \lesssim\|\mathbf{v}\|_{E}, \quad \forall \mathbf{v} \in \mathbf{V}_{h, i} .
$$

Furthermore, due to (3.15) and (3.20),

$$
\Pi^{R T} \Pi \mathbf{q}=\Pi^{R T} \mathbf{q}
$$

\subsection{A quadrature rule}

Recall the variational formulation (2.3)-(2.4). In its mixed finite element discretization, one needs to compute the integral $\left(K^{-1} \mathbf{q}, \mathbf{v}\right)_{\Omega_{i}}$ for $\mathbf{q}, \mathbf{v} \in \mathbf{V}_{h, i}$. The MFMFE method employs a quadrature rule for the velocity mass matrix, in order to reduce the discrete problem on each subdomain to a cell-centered finite difference system for the pressure. We follow the development in $[39,56]$. The integration on each element $E$ is performed by mapping to the reference element $\hat{E}$, where the quadrature rule is defined. Using the definition (3.14) of the finite element spaces, for $\mathbf{q}, \mathbf{v} \in \mathbf{V}_{h, i}$,

$$
\left(K^{-1} \mathbf{q}, \mathbf{v}\right)_{E}=\left(\frac{1}{J_{E}} D F_{E}^{T} K^{-1}\left(F_{E}(\hat{x})\right) D F_{E} \hat{\mathbf{q}}, \hat{\mathbf{v}}\right)_{\hat{E}} \equiv\left(\mathcal{K}^{-1} \hat{\mathbf{q}}, \hat{\mathbf{v}}\right)_{\hat{E}}
$$

where

$$
\mathcal{K}^{-1}(\hat{x})=\frac{1}{J_{E}} D F_{E}^{T} K^{-1}\left(F_{E}(\hat{x})\right) D F_{E}
$$

Due to (3.6), we have

$$
\left\|\mathcal{K}^{-1}\right\|_{0, \infty, \hat{E}} \bar{\sim} h^{2-d}\left\|K^{-1}\right\|_{0, \infty, E} .
$$

The quadrature rule on an element $E$ is defined by the trapezoidal rule:

$$
\left(K^{-1} \mathbf{q}, \mathbf{v}\right)_{Q, E}=\left(\mathcal{K}^{-1} \hat{\mathbf{q}}, \hat{\mathbf{v}}\right)_{\hat{Q}, \hat{E}} \equiv \frac{|\hat{E}|}{n_{v}} \sum_{i=1}^{n_{v}} \mathcal{K}^{-1}\left(\hat{\mathbf{r}}_{i}\right) \hat{\mathbf{q}}\left(\hat{\mathbf{r}}_{i}\right) \cdot \hat{\mathbf{v}}\left(\hat{\mathbf{r}}_{i}\right),
$$

where $n_{v}$ is the number of vertices of $\hat{E}$. The global quadrature rule on $\Omega_{i}$ is defined as

$$
\left(K^{-1} \mathbf{q}, \mathbf{v}\right)_{Q, \Omega_{i}} \equiv \sum_{E \in \mathcal{T}_{h, i}}\left(K^{-1} \mathbf{q}, \mathbf{v}\right)_{Q, E}
$$

The corner vector $\hat{\mathbf{q}}\left(\hat{\mathbf{r}}_{i}\right)$ is uniquely determined by its normal components to the $d$ faces that share the vertex. Since we chose the velocity degrees freedom associated with each corner $\hat{\mathbf{r}}_{i}$, the $d$ degrees of freedom associated with each corner $\hat{\mathbf{r}}_{i}$ uniquely determine the corner vector $\hat{\mathbf{q}}\left(\hat{\mathbf{r}}_{i}\right)$. More precisely,

$$
\hat{\mathbf{q}}\left(\hat{\mathbf{r}}_{i}\right)=\sum_{j=1}^{d}\left(\hat{\mathbf{q}} \cdot \hat{\mathbf{n}}_{i j}\right)\left(\hat{\mathbf{r}}_{i}\right) \hat{\mathbf{n}}_{i j},
$$


where $\hat{\mathbf{n}}_{i j}, j=1, \ldots, d$, are the outward unit normal vectors to the $d$ faces (or edges) sharing $\hat{\mathbf{r}}_{i}$, and $\left(\hat{\mathbf{q}} \cdot \hat{\mathbf{n}}_{i j}\right)\left(\hat{\mathbf{r}}_{i}\right)$ are the velocity degrees of freedom associated with this corner. Denote the basis functions associated with $\hat{\mathbf{r}}_{i}$ by $\hat{\mathbf{v}}_{i j}, j=1, \ldots, d$ :

$$
\left(\hat{\mathbf{v}}_{i j} \cdot \hat{\mathbf{n}}_{i j}\right)\left(\hat{\mathbf{r}}_{i}\right)=1, \quad\left(\hat{\mathbf{v}}_{i j} \cdot \hat{\mathbf{n}}_{i k}\right)\left(\hat{\mathbf{r}}_{i}\right)=0, k \neq j, \quad \text { and }\left(\hat{\mathbf{v}}_{i j} \cdot \hat{\mathbf{n}}_{l k}\right)\left(\hat{\mathbf{r}}_{l}\right)=0, l \neq i, k=1, \ldots, d .
$$

The quadrature rule (3.28) couples only the $d$ basis functions associated with a corner. For example, on the unit cube,

$$
\begin{aligned}
& \left(\mathcal{K}^{-1} \hat{\mathbf{v}}_{11}, \hat{\mathbf{v}}_{11}\right)_{\hat{Q}, \hat{E}}=\frac{\mathcal{K}_{11}^{-1}\left(\hat{\mathbf{r}}_{1}\right)}{8}, \quad\left(\mathcal{K}^{-1} \hat{\mathbf{v}}_{11}, \hat{\mathbf{v}}_{12}\right)_{\hat{Q}, \hat{E}}=\frac{\mathcal{K}_{21}^{-1}\left(\hat{\mathbf{r}}_{1}\right)}{8}, \\
& \left(\mathcal{K}^{-1} \hat{\mathbf{v}}_{11}, \hat{\mathbf{v}}_{13}\right)_{\hat{Q}, \hat{E}}=\frac{\mathcal{K}_{31}^{-1}\left(\hat{\mathbf{r}}_{1}\right)}{8}, \quad \text { and }\left(\mathcal{K}^{-1} \hat{\mathbf{v}}_{11}, \hat{\mathbf{v}}_{i j}\right)_{\hat{Q}, \hat{E}}=0 \quad i \neq 1, j=1,2,3 .
\end{aligned}
$$

Mapping back to the physical element $E$, we have

$$
\left(K^{-1} \mathbf{q}, \mathbf{v}\right)_{Q, E}=\frac{1}{n_{v}} \sum_{i=1}^{n_{v}} J_{E}\left(\hat{\mathbf{r}}_{i}\right) K^{-1}\left(\mathbf{r}_{i}\right) \mathbf{q}\left(\mathbf{r}_{i}\right) \cdot \mathbf{v}\left(\mathbf{r}_{i}\right),
$$

which is closely related to an inner products used in the mimetic finite difference methods [38].

It has been shown $[39,56]$ that the bilinear form $\left(K^{-1} \mathbf{q}, \mathbf{v}\right)_{Q, \Omega_{i}}$ is an inner product in $\mathbf{V}_{h, i}$ and $\left(K^{-1} \mathbf{q}, \mathbf{q}\right)_{Q, \Omega_{i}}^{1 / 2}$ is a norm equivalent to $\|\cdot\|_{\Omega_{i}}$ :

$$
\left(K^{-1} \mathbf{q}, \mathbf{q}\right)_{Q, \Omega_{i}} \approx\|\mathbf{q}\|_{\Omega_{i}}^{2} \quad \forall \mathbf{q} \in \mathbf{V}_{h, i}
$$

In the pressure superconvergence analysis, we need additional property of the bilinear form $\left(K^{-1}, \cdot\right)_{Q, \Omega_{i}}$ on the space

$$
\overline{\mathbf{V}}_{h, i}:=\mathbf{V}_{h, i} \bigcup \mathbf{W}_{h, i}
$$

where $\mathbf{W}_{h, i}$ is the space of piecewise constant vectors defined element by element.

Lemma 3.1. The bilinear form $\left(K^{-1} \cdot, \cdot\right)_{Q, \Omega_{i}}$ is an inner product on $\overline{\mathbf{V}}_{h, i}$ and the norm $\left(K^{-1} \mathbf{q}, \mathbf{q}\right)_{Q, \Omega_{i}}^{1 / 2}$ on $\overline{\mathbf{V}}_{h, i}$ is equivalent to $\|\cdot\|_{\Omega_{i}}$ :

$$
\left(K^{-1} \mathbf{q}, \mathbf{q}\right)_{Q, \Omega_{i}}^{1 / 2} \bar{\sim}\|\mathbf{q}\|_{\Omega_{i}}, \quad \forall \mathbf{q} \in \overline{\mathbf{V}}_{h, i}
$$

Proof. On each element $E$, let $\mathbf{q}=\sum_{i=1}^{n_{v}} \sum_{j=1}^{d} q_{i j} \mathbf{v}_{i j}+\mathbf{q}_{0}$ where $\mathbf{v}_{i j}$ are the normalized basis functions of $\mathbf{V}_{h, i}(E)$ and $\mathbf{q}_{0}$ is a constant vector. Using (3.30), (1.4), (3.6), we have

$$
\left(K^{-1} \mathbf{q}, \mathbf{q}\right)_{Q, E} \gtrsim \frac{|E|}{k_{1}}\left(\sum_{i=1}^{n_{v}} \sum_{j=1}^{d} q_{i j}^{2}+\left|\mathbf{q}_{0}\right|^{2}\right) .
$$

This gives that $\left(K^{-1} \cdot, \cdot\right)_{Q, E}$ is an inner product. Using (3.27), the fact that $\|\mathbf{q}\|_{E}^{2} \bar{\sim} h^{2-d}\|\hat{\mathbf{q}}\|_{\hat{E}}^{2}$ for all $\mathbf{q} \in$ $\left(L^{2}(E)\right)^{d}$ (see Lem. 2.3 in [56]), and norm equivalence on the reference element $\hat{E}$, we obtain for all $\mathbf{q} \in \overline{\mathbf{V}}_{h}$,

$$
\left(K^{-1} \mathbf{q}, \mathbf{q}\right)_{Q, E}=\left(\mathcal{K}^{-1} \hat{\mathbf{q}}, \hat{\mathbf{q}}\right)_{\hat{Q}, \hat{E}} \approx h^{2-d}\|\hat{\mathbf{q}}\|_{\hat{E}}^{2} \approx\|\mathbf{q}\|_{E}^{2} .
$$

The numerical quadrature error on each element is defined as

$$
\sigma_{E}(\mathbf{q}, \mathbf{v}) \equiv(\mathbf{q}, \mathbf{v})_{E}-(\mathbf{q}, \mathbf{v})_{Q, E},
$$

and the global numerical quadrature error is given by $\left.\sigma(\mathbf{q}, \mathbf{v})\right|_{E} \equiv \sigma_{E}(\mathbf{q}, \mathbf{v})$. 

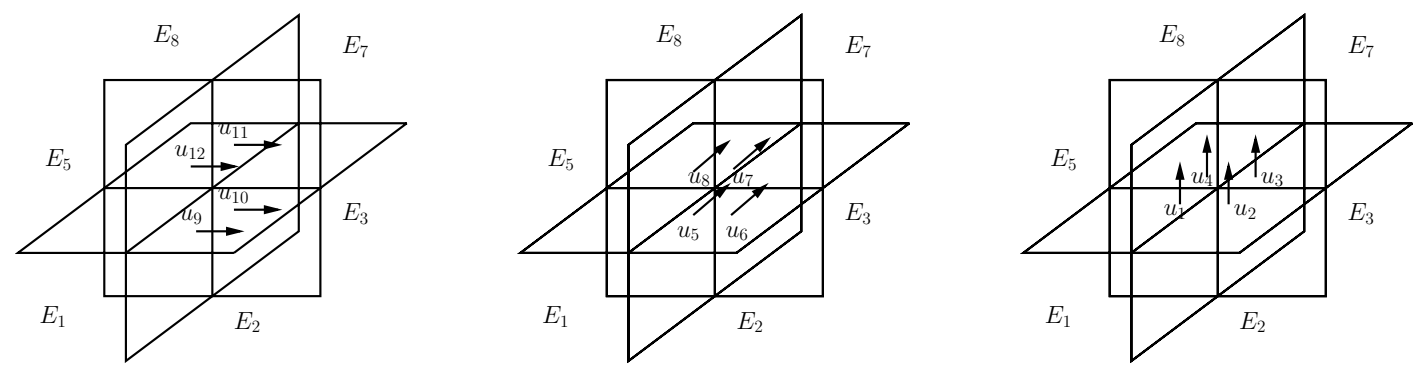

FIGURE 2. Interactions of the velocity degrees of freedom in the MFMFE method.

\subsection{Reduction to a cell centered finite difference system for the pressure}

We next describe how the quadrature rule for the velocity mass matrix allows one to reduce the MFMFE method on each subdomain to a centered finite difference system for the pressure. We limit the discussion to hexahedral grids; the other element types are treated similarly. We refer to Figure 2 for the notation used in this section. Any interior vertex $\mathbf{r}$ is shared by 8 elements $E_{1}, \ldots, E_{8}$. We denote the faces that share the vertex by $e_{1}, \ldots, e_{12}$, and the velocity basis functions on these faces that are associated with the vertex by $\mathbf{v}_{1}, \ldots, \mathbf{v}_{12}$, i.e., $\left(\mathbf{v}_{i} \cdot \mathbf{n}_{i}\right)(\mathbf{r})=1$, where $\mathbf{n}_{i}$ is the unit normal on face $e_{i}$. The corresponding values of the normal components of $\mathbf{u}_{h}, u_{1}, \ldots, u_{12}$ are depicted in the three images in directions $x, y$, and $z$, respectively.

Recall that the quadrature rule $\left(K^{-1} \cdot, \cdot\right)_{Q}$ localizes the basis functions interaction, see (3.29). Therefore, taking $\mathbf{v}=\mathbf{v}_{1}$ in $\left(K^{-1} \mathbf{u}_{h}, \cdot\right)_{Q}$, for example, will lead to coupling $u_{1}$ only with $u_{5}, u_{8}, u_{9}$, and $u_{12}$. Similarly, $u_{2}$ will be coupled only with $u_{6}, u_{7}, u_{9}$, and $u_{12}$, etc. Therefore, the 12 equations obtained from taking $\mathbf{v}=\mathbf{v}_{1}, \ldots, \mathbf{v}_{12}$ form a linear system for $u_{1}, \ldots, u_{12}$. The following result is a direct consequence of (3.31).

Proposition 3.2. The $12 \times 12$ local linear system described above is symmetric and positive definite.

The solution of the local $12 \times 12$ linear system allows for the velocities $u_{i}, i=1, \ldots, 12$ to be expressed in terms of the cell-centered pressures $p_{i}, i=1, \ldots, 8$. Substituting these expressions into the mass conservation equation (4.2) leads to a cell-centered stencil. The pressure in each element $E$ is coupled with the pressures in the elements that share a vertex with $E$, leading to a 27 point stencil. The reader is referred to [39, 56] for further details on the resulting cell-centered finite difference system.

\section{Multiscale mortar multipoint FluX miXed finite Element Method}

Define the global mesh partition on $\Omega$ as $\mathcal{T}_{h}=\bigoplus_{i=1}^{n} \mathcal{T}_{h, i}$ and the finite element spaces on $\Omega$ as

$$
\mathbf{V}_{h}=\bigoplus_{i=1}^{n} \mathbf{V}_{h, i}, \quad \mathbf{V}_{h}^{R T}=\bigoplus_{i=1}^{n} \mathbf{V}_{h, i}^{R T}, \quad W_{h}=\bigoplus_{i=1}^{n} W_{h, i} .
$$

Let the mortar interface mesh $\mathcal{T}_{H, i, j}$ be a quasi-uniform partition of $\Gamma_{i, j}$, with maximal element diameter $H_{i, j}$. Let $H=\max _{1 \leq i, j \leq n} H_{i, j}$. Denote by $\Lambda_{H, i, j} \subset L^{2}\left(\Gamma_{i, j}\right)$ the mortar space on $\Gamma_{i, j}$, containing either continuous or discontinuous piecewise polynomials of degree $m$ on $\mathcal{T}_{H, i, j}$. Note that $\mathcal{T}_{H, i, j}$ need not be conforming if $\Lambda_{H, i, j}$ is a discontinuous space. Let

$$
\Lambda_{H}=\bigoplus_{1 \leq i<j \leq n} \Lambda_{H, i, j}
$$

be the mortar finite element space on $\Gamma$. 
The multiscale mortar MFMFE method on quadrilaterals and hexahedra is defined as: seek $\mathbf{u}_{h} \in \mathbf{V}_{h}, p_{h} \in$ $W_{h}$, and $\lambda_{H} \in \Lambda_{H}$ such that, for $1 \leq i \leq n$,

$$
\begin{aligned}
\left(K^{-1} \mathbf{u}_{h}, \mathbf{v}\right)_{Q, \Omega_{i}}-\left(p_{h}, \nabla \cdot \mathbf{v}\right)_{\Omega_{i}} & =-\left\langle\lambda_{H}, \Pi^{R T} \mathbf{v} \cdot \mathbf{n}_{i}\right\rangle_{\Gamma_{i}} & & \\
& -\left\langle g, \Pi^{R T} \mathbf{v} \cdot \mathbf{n}_{i}\right\rangle_{\partial \Omega_{i} \backslash \Gamma}, & & \forall \mathbf{v} \in \mathbf{V}_{h, i}, \\
\left(\nabla \cdot \mathbf{u}_{h}, w\right)_{\Omega_{i}} & =(f, w)_{\Omega_{i}}, & & \forall w \in W_{h, i}, \\
\sum_{i=1}^{n}\left\langle\Pi^{R T} \mathbf{u}_{h} \cdot \mathbf{n}_{i}, \mu\right\rangle_{\Gamma_{i}} & =0, & & \forall \mu \in \Lambda_{H},
\end{aligned}
$$

where $\Gamma_{i}=\partial \Omega_{i} \backslash \partial \Omega$. The multiscale mortar MFMFE method on simplicial elements is defined as above, except for removing the projection operator $\Pi^{R T}$ in (4.1) and (4.3).

Within each block $\Omega_{i}$, we have the MFMFE method based on the quadrature rule described in Section 3, where (4.2) gives local conservation over each fine grid element. Equation (4.3) enforces a weak continuity of flux across the interfaces with respect to the mortar space $\Lambda_{H}$.

Similar methods on affine mixed finite elements have been introduced and analyzed. In [11], the mortar mesh size $H$ is comparable to $h$ and the mortar degree of polynomial is one order higher than the degree of approximation polynomials in $\mathbf{V}_{h}$. In [12], the discretization of $\Gamma$ is weakened by allowing larger elements of size $H$ but considering higher degree of mortar approximation. In the present work, we consider the MFMFE method as a subdomain discretization on affine, quadrilateral, and hexahedral grids. The convergence and choice of mortar degrees are similar to the $\mathrm{RT}_{0}$ case in [12].

Remark 4.1. The appearance of $\Pi^{R T}$ in (4.3) in the case of quadrilaterals and hexahedra is not standard. It is necessary to have $\Pi^{R T}$ in (4.1) for accuracy. More precisely, the numerical quadrature error can only be controlled when one of the arguments belongs to $\mathbf{V}_{h}^{R T}$, see Lemma 6.3. Having $\Pi^{R T}$ in (4.3) maintains the symmetry of the method and also guarantees existence and uniqueness of a solution of (4.1)-(4.3) as shown in Lemma 6.1. On the other hand, in the case of simplicial elements, Lemma 6.3 holds also when the arguments are in $\mathbf{V}_{h}$, so there is no need to project the mortar and boundary data into $\mathbf{V}_{h}^{R T}$. In fact, such projection would lead to a non-convergent method, since Lemma 6.2 does not hold on simplices.

In the next three sections we state the results for all element types (except Sect. 6.3 where only rectangular and cuboid grids are considered for the velocity superconvergence), but present the arguments only for quadrilaterals and hexahedra. The arguments for simplicial elements are the same, except that the superscript $R T$ needs to be removed from all of its appearances. In particular, replace $\Pi^{R T}$ by $\Pi$, which means removing $\Pi^{R T}$, since it always acts on vectors in $\mathbf{V}_{h}, Q_{h}^{R T}$ by $Q_{h}, \mathbf{V}_{h}^{R T}$ by $\mathbf{V}_{h}$, etc. As a result, terms of the type $\mathbf{v}-\Pi^{R T} \mathbf{v}$ drop out. We note that Lemma 6.2 is not needed in the argument for simplicial elements, since the term in question does not appear in this case.

\section{Approximation Properties}

\subsection{Element geometry}

For the convergence analysis we need some restrictions on the element geometry. This is due to the reduced approximation property of the MFE spaces on general quadrilateral and hexahedra. Numerical examples confirm that the restrictions are not just for theoretical purposes $[5,39,56]$. Following the terminology in $[39,56]$, we introduce the following definitions.

Definition 5.1. The (possibly non-planar) faces of a hexahedral element $E$ defined via a trilinear mapping in three dimensions are called generalized quadrilaterals. 
Definition 5.2. A generalized quadrilateral with vertices $\mathbf{r}_{1}, \ldots, \mathbf{r}_{4}$ is called an $h^{2}$-parallelogram if

$$
\left|\mathbf{r}_{34}-\mathbf{r}_{21}\right|_{\mathbb{R}^{d}} \lesssim h^{2}
$$

where $|\cdot|_{\mathbb{R}^{d}}$ is the Euclidean norm in $\mathbb{R}^{d}$.

Definition 5.3. A hexahedral element is called a $h^{2}$-parallelepiped if all of its faces are $h^{2}$-parallelograms.

Definition 5.4. An $h^{2}$-parallelepiped is called regular if

$$
\left|\left(\mathbf{r}_{21}-\mathbf{r}_{34}\right)-\left(\mathbf{r}_{65}-\mathbf{r}_{78}\right)\right|_{\mathbb{R}^{d}} \lesssim h^{3} .
$$

\subsection{MFE approximation properties}

We state several approximation properties of the MFE projection operators. On simplicial, $h^{2}$-parallelogram, and $h^{2}$-parallelepiped grids, the following bounds hold on any element $E$ :

$$
\begin{aligned}
& \left\|\mathbf{q}-\Pi^{R T} \mathbf{q}\right\|_{E}+\|\mathbf{q}-\Pi \mathbf{q}\|_{E} \lesssim h\|\mathbf{q}\|_{1, E}, \\
& \left\|\nabla \cdot\left(\mathbf{q}-\Pi^{R T} \mathbf{q}\right)\right\|_{E}+\|\nabla \cdot(\mathbf{q}-\Pi \mathbf{q})\|_{E} \lesssim h\|\nabla \cdot \mathbf{q}\|_{1, E} .
\end{aligned}
$$

The above bounds can be found in [19,49] for simplicial elements, [14,54] for $h^{2}$-parallelograms, and [39] for $h^{2}$ parallelepipeds. A higher order approximation property also holds for simplicial, $h^{2}$-parallelogram, and regular $h^{2}$-parallelepiped grids:

$$
\|\mathbf{q}-\Pi \mathbf{q}\|_{E} \lesssim h^{2}\|\mathbf{q}\|_{2, E} .
$$

On general quadrilaterals, bound (5.2) is also valid [14]. However, in this case for the divergence bound it only holds for

$$
\|\nabla \cdot(\mathbf{q}-\Pi \mathbf{q})\|_{E} \lesssim\|\nabla \cdot \mathbf{q}\|_{E} .
$$

The following lemma has been shown in $[39,56]$.

Lemma 5.5. For all elements E,

$$
\|\Pi \mathbf{q}\|_{j, E} \lesssim\|\mathbf{q}\|_{j, E}, \quad \forall \mathbf{q} \in H^{j}(E)^{d}
$$

holds for $j=1,2$ on simplicial elements, $h^{2}$-parallelograms, and regular $h^{2}$-parallelepipeds, as well as $j=1$ on $h^{2}$-parallelepipeds. Furthermore, on simplicial elements, $h^{2}$-parallelograms, and $h^{2}$-parallelepipeds,

$$
\left\|\Pi^{R T} \mathbf{q}\right\|_{1, E} \lesssim\|\mathbf{q}\|_{1, E}, \quad \forall \mathbf{q} \in H^{1}(E)^{d} .
$$

For the remainder of the paper, we assume that the quadrilateral elements are $h^{2}$-parallelograms, and the hexahedral elements are $h^{2}$-parallelepipeds. We also need the regular $h^{2}$-parallelepiped condition for the pressure superconvergence bound in Section 6.5.

Let $\hat{Q}$ be the $L^{2}(\hat{E})$-orthogonal projection onto $\hat{W}(\hat{E})$, satisfying for any $\hat{\varphi} \in L^{2}(\hat{E})$,

$$
(\hat{\varphi}-\hat{Q} \hat{\varphi}, \hat{w})_{\hat{E}}=0, \quad \forall \hat{w} \in \hat{W}(\hat{E}) .
$$

Let $\mathcal{Q}_{h}: L^{2}(\Omega) \rightarrow W_{h}$ be the projection operator satisfying for any $\varphi \in L^{2}(\Omega)$,

$$
\mathcal{Q}_{h} \varphi=\hat{Q} \hat{\varphi} \circ F_{E}^{-1} \quad \text { on all } E .
$$

It is easy to see that, due to (3.11),

$$
\left(\varphi-\mathcal{Q}_{h} \varphi, \nabla \cdot \mathbf{v}\right)=0, \quad \forall \mathbf{v} \in \mathbf{V}_{h}
$$


Using a scaling argument and the Bramble-Hilbert lemma, it can be shown that

$$
\left\|\varphi-\mathcal{Q}_{h} \varphi\right\| \lesssim h|\varphi|_{1} .
$$

We also need approximation properties for the finite element spaces on the interfaces. Let $\mathcal{I}_{H}^{c}$ be the ScottZhang operator [51] onto the space $\Lambda_{H}^{c}$, the subset of continuous functions in $\Lambda_{H}$. Similarly, let $\mathcal{P}_{H}$ denote $L^{2}(\Gamma)$ projection operator onto $\Lambda_{H}$. For each subdomain $\Omega_{i}$, define a projection operator $\mathcal{Q}_{h, i}^{R T}:\left.L^{2}\left(\Gamma_{i}\right) \rightarrow \mathbf{V}_{h, i}^{R T} \cdot \mathbf{n}_{i}\right|_{\Gamma_{i}}$ such that for any $\varphi \in L^{2}\left(\Gamma_{i}\right)$

$$
\left\langle\varphi-\mathcal{Q}_{h, i}^{R T} \varphi, \mathbf{v} \cdot \mathbf{n}_{i}\right\rangle_{\Gamma_{i}}=0, \quad \forall \mathbf{v} \in V_{h, i}^{R T}
$$

Due to (3.20),

$$
\left(\Pi^{R T} \mathbf{q}\right) \cdot \mathbf{n}_{i}=\mathcal{Q}_{h, i}^{R T}\left(\mathbf{q} \cdot \mathbf{n}_{i}\right) .
$$

The above defined interface operators have the following approximation properties [25, 51]:

$$
\begin{array}{lr}
\left\|\phi-\mathcal{I}_{H}^{c} \phi\right\|_{t, \Gamma_{i, j}} \lesssim H^{s-t}\|\phi\|_{s, \Gamma_{i, j}}, & 0 \leq s \leq m+1, \quad 0 \leq t \leq \min (1, s), \\
\left\|\phi-\mathcal{P}_{H} \phi\right\|_{-t, \Gamma_{i, j}} \lesssim H^{s+t}\|\phi\|_{s, \Gamma_{i, j}}, & 0 \leq s \leq m+1, \quad 0 \leq t \leq 1, \\
\left\|\left(\mathbf{q}-\Pi^{R T} \mathbf{q}\right) \cdot \mathbf{n}_{i}\right\|_{-t, \Gamma_{i, j}} \lesssim h^{r+t}\left\|\mathbf{q} \cdot \mathbf{n}_{i}\right\|_{r, \Gamma_{i, j}}, & 0 \leq r \leq 1, \quad 0 \leq t \leq 1,
\end{array}
$$

where $\|\cdot\|_{-t}$ is the norm of $H^{-t}$, the dual of $H^{t}$.

\subsection{The spaces of weakly continuous velocities}

Define a weakly continuous BDM velocity in $2 \mathrm{D}$ and enhanced BDDF velocity space in $3 \mathrm{D}$ as

$$
\mathbf{V}_{h, 0}=\left\{\mathbf{v} \in \mathbf{V}_{h}: \sum_{i=1}^{n}\left\langle\left.\Pi^{R T} \mathbf{v}\right|_{\Omega_{i}} \cdot \mathbf{n}_{i}, \mu\right\rangle_{\Gamma_{i}}=0 \quad \forall \mu \in \Lambda_{H}\right\} .
$$

The multiscale mortar MFMFE method (4.1)-(4.3) is equivalent to the following problem: find $\mathbf{u}_{h} \in \mathbf{V}_{h, 0}$ and $p_{h} \in W_{h}$ such that

$$
\begin{array}{cl}
\left(K^{-1} \mathbf{u}_{h}, \mathbf{v}\right)_{Q}-\sum_{i=1}^{n}\left(p_{h}, \nabla \cdot \mathbf{v}\right)_{\Omega_{i}}=-\left\langle g, \Pi^{R T} \mathbf{v} \cdot \mathbf{n}_{i}\right\rangle_{\partial \Omega_{i} / \Gamma}, & \forall \mathbf{v} \in \mathbf{V}_{h, 0}, \\
\sum_{i=1}^{n}\left(\nabla \cdot \mathbf{u}_{h}, w\right)_{\Omega_{i}}=(f, w), & \forall w \in W_{h} .
\end{array}
$$

We will also make use of the RT weakly continuous space

$$
\mathbf{V}_{h, 0}^{R T}=\left\{\mathbf{v} \in \mathbf{V}_{h}^{R T}: \sum_{i=1}^{n}\left\langle\left.\mathbf{v}\right|_{\Omega_{i}} \cdot \mathbf{n}_{i}, \mu\right\rangle_{\Gamma_{i}}=0 \quad \forall \mu \in \Lambda_{H}\right\} .
$$

The following lemma has been shown in [12].

Lemma 5.6. Assume that for any $\mu \in \Lambda_{H}$,

$$
\|\mu\|_{\Gamma_{i, j}} \leq C\left(\left\|\mathcal{Q}_{h, i}^{R T} \mu\right\|_{\Gamma_{i, j}}+\left\|\mathcal{Q}_{h, j}^{R T} \mu\right\|_{\Gamma_{i, j}}\right), \quad 1 \leq i<j \leq n .
$$

Then there exists a projection operator $\Pi_{0}^{R T}:\left(H^{1 / 2+\epsilon}(\Omega)\right)^{d} \cap \mathbf{V} \rightarrow \mathbf{V}_{h, 0}^{R T}$ such that

$$
\begin{aligned}
& \left(\nabla \cdot\left(\Pi_{0}^{R T} \mathbf{q}-\mathbf{q}\right), w\right)_{\Omega_{i}}=0, \quad w \in W_{h}, \quad 1 \leq i \leq n, \\
& \left\|\Pi_{0}^{R T} \mathbf{q}-\Pi^{R T} \mathbf{q}\right\| \lesssim\|\mathbf{q}\|_{r+1 / 2} h^{r} H^{1 / 2}, \quad 0<r \leq 1, \\
& \left\|\Pi_{0}^{R T} \mathbf{q}-\mathbf{q}\right\| \lesssim \sum_{i=1}^{n}\|\mathbf{q}\|_{1, \Omega_{i}} h+\|\mathbf{q}\|_{r+1 / 2} h^{r} H^{1 / 2}, \quad 0<r \leq 1 .
\end{aligned}
$$


Remark 5.7. Condition (5.18) states that the mortar space cannot be too rich compared to the normal traces of the subdomain velocity spaces. It is not very restrictive, and is easily satisfied in practice, especially for multiscale discretizations with coarse scale mortars. Some practical criteria for satisfying (5.18) are given in [47].

In the following we construct a projection operator $\Pi_{0}$ into $\mathbf{V}_{h, 0}$ with similar properties.

By an abuse of notation, define

$$
\begin{aligned}
& \mathbf{V}_{h, 0}^{R T} \cdot \mathbf{n}=\left\{\left(\phi_{L}, \phi_{R}\right) \in L^{2}(\Gamma) \times L^{2}(\Gamma): \quad \exists \mathbf{v} \in \mathbf{V}_{h, 0}^{R T}\right. \text { such that } \\
&\left.\left.\phi_{L}\right|_{\Gamma_{i, j}}=\left.\mathbf{v}\right|_{\Omega_{i}} \cdot \mathbf{n}_{i} \quad \text { and }\left.\phi_{R}\right|_{\Gamma_{i, j}}=\left.\mathbf{v}\right|_{\Omega_{j}} \cdot \mathbf{n}_{j} \quad \forall 1 \leq i<j \leq n\right\} .
\end{aligned}
$$

Then, for any $\phi=\left(\phi_{L}, \phi_{R}\right) \in\left(L^{2}(\Gamma)\right)^{2}$, we write $\left.\phi\right|_{\Gamma_{i, j}}=\left(\phi_{i}, \phi_{j}\right), 1 \leq i<j \leq n$. Define a projection operator $\mathcal{Q}_{h, 0}^{R T}:\left(L^{2}(\Gamma)\right)^{2} \rightarrow \mathbf{V}_{h, 0}^{R T} \cdot \mathbf{n}$ such that, for any $\phi \in\left(L^{2}(\Gamma)\right)^{2}$,

$$
\sum_{i=1}^{n}\left\langle\phi_{i}-\left(\mathcal{Q}_{h, 0}^{R T} \phi\right)_{i}, \eta_{i}\right\rangle_{\Gamma_{i}}=0, \quad \forall \eta \in \mathbf{V}_{h, 0}^{R T} \cdot \mathbf{n}
$$

The proof of the following two lemmas can be found in [11], Section 3, with a straightforward modification of the argument for the two scales $h$ and $H$.

Lemma 5.8. Assume that for any $\mu \in \Lambda_{H}$,

$$
\mathcal{Q}_{h, i}^{R T} \mu=0, \quad 1 \leq i \leq n, \quad \text { implies that } \mu=0 .
$$

Then, for any $\phi \in\left(L^{2}(\Gamma)\right)^{2}$, there exists $\lambda_{H} \in \Lambda_{H}$ such that on $\Gamma_{i, j}, 1 \leq i \leq j \leq n$,

$$
\mathcal{Q}_{h, i}^{R T} \lambda_{H}=\mathcal{Q}_{h, i}^{R T} \phi_{i}-\left(\mathcal{Q}_{h, 0}^{R T} \phi\right)_{i}, \quad \mathcal{Q}_{h, j}^{R T} \lambda_{H}=\mathcal{Q}_{h, j}^{R T} \phi_{j}-\left(\mathcal{Q}_{h, 0}^{R T} \phi\right)_{j}, \quad \text { and } \quad\left\langle\lambda_{H}, 1\right\rangle_{\Gamma_{i, j}}=\frac{1}{2}\left\langle\phi_{i}+\phi_{j}, 1\right\rangle_{\Gamma_{i, j}} .
$$

Lemma 5.9. Under assumption (5.18), for any $\left.\phi\right|_{\Gamma_{i, j}}=\left(\phi_{i},-\phi_{i}\right)$,

$$
\left(\sum_{1 \leq i<j \leq n}\left\|\mathcal{Q}_{h, i}^{R T} \phi_{i}-\left(\mathcal{Q}_{h, 0}^{R T} \phi\right)_{i}\right\|_{-s, \Gamma_{i, j}}^{2}\right)^{1 / 2} \lesssim \sum_{1 \leq i<j \leq n}\left\|\phi_{i}\right\|_{r, \Gamma_{i, j}} h^{r} H^{s}, \quad 0 \leq r \leq 1,0 \leq s \leq 1 .
$$

We are now ready to construct a projection operator $\Pi_{0}$ onto $\mathbf{V}_{h, 0}$. Our approach is similar to the one in [11]. For any $\mathbf{q} \in\left(H^{1 / 2+\epsilon}(\Omega)\right)^{d} \cap \mathbf{V}, \epsilon>0$, define

$$
\left.\Pi_{0} \mathbf{q}\right|_{\Omega_{i}}=\Pi\left(\mathbf{q}+\delta \mathbf{q}_{i}\right),
$$

where $\delta \mathbf{q}_{i}$ solves

$$
\begin{array}{lr}
\delta \mathbf{q}_{i}=-\nabla \pi_{i} & \text { in } \Omega_{i}, \\
\nabla \cdot \delta \mathbf{q}_{i}=0 & \text { in } \Omega_{i}, \\
\delta \mathbf{q}_{i} \cdot \mathbf{n}_{i}=-\mathcal{Q}_{h, i}^{R T} \mathbf{q} \cdot \mathbf{n}_{i}+\left(\mathcal{Q}_{h, 0}^{R T} \mathbf{q} \cdot \mathbf{n}\right)_{i} & \text { on } \Gamma_{i}, \\
\delta \mathbf{q}_{i} \cdot \mathbf{n}_{i}=0 & \text { on } \partial \Omega_{i} \cap \partial \Omega,
\end{array}
$$

where $\left.\mathbf{q} \cdot \mathbf{n}\right|_{\Gamma_{i, j}}=\left(\mathbf{q} \cdot \mathbf{n}_{i}, \mathbf{q} \cdot \mathbf{n}_{j}\right)$. The Neumann problem (5.26)-(5.29) is solvable, since (5.24) implies the solvability condition

$$
\left\langle\mathcal{Q}_{h, i}^{R T} \mathbf{q} \cdot \mathbf{n}_{i}-\left(\mathcal{Q}_{h, 0}^{R T} \mathbf{q} \cdot \mathbf{n}\right)_{i}, 1\right\rangle_{\Gamma_{i}}=0
$$


By the construction of $\Pi_{0}$ and using (3.25), for all $\mu \in \Lambda_{H}$,

$$
\begin{aligned}
& \sum_{i=1}^{n}\langle\left.\Pi^{R T}\left(\Pi_{0} \mathbf{q}\right) \cdot \mathbf{n}_{i}, \mu\right\rangle_{\Gamma_{i}}=\sum_{i=1}^{n}\left\langle\Pi^{R T}\left(\mathbf{q}+\delta \mathbf{q}_{i}\right) \cdot \mathbf{n}_{i}, \mu\right\rangle_{\Gamma_{i}} \\
&=\sum_{i=1}^{n}\left\langle\mathcal{Q}_{h, i}^{R T}\left(\mathbf{q}+\delta \mathbf{q}_{i}\right) \cdot \mathbf{n}_{i}, \mu\right\rangle_{\Gamma_{i}}=\sum_{i=1}^{n}\left\langle\left(\mathcal{Q}_{h, 0}^{R T} \mathbf{q} \cdot \mathbf{n}\right)_{i}, \mu\right\rangle_{\Gamma_{i}}=0
\end{aligned}
$$

where we have used (5.10) and (5.28). Thus, $\Pi_{0} \mathbf{q} \in \mathbf{V}_{h, 0}$. Also,

$$
\left(\nabla \cdot \Pi_{0} \mathbf{q}, w\right)_{\Omega_{i}}=(\nabla \cdot \Pi \mathbf{q}, w)_{\Omega_{i}}+\left(\nabla \cdot \Pi \delta \mathbf{q}_{i}, w\right)_{\Omega_{i}}=(\nabla \cdot \mathbf{q}, w)_{\Omega_{i}}, \quad \forall w \in W_{h, i}
$$

It remains to estimate the approximability of $\Pi_{0}$. Since $\Pi_{0} \mathbf{q}-\Pi \mathbf{q}=\Pi \delta \mathbf{q}_{i}$, we only need to bound the correction term $\Pi \delta \mathbf{q}_{i}$. By elliptic regularity [35,43], for any $0 \leq s \leq 1 / 2$,

$$
\left\|\delta \mathbf{q}_{i}\right\|_{1 / 2-s, \Omega_{i}} \lesssim \sum_{j}\left\|\mathcal{Q}_{h, i}^{R T} \mathbf{q} \cdot \mathbf{n}_{i}-\left(\mathcal{Q}_{h, 0}^{R T} \mathbf{q} \cdot \mathbf{n}\right)_{i}\right\|_{-s, \Gamma_{i, j}}
$$

Then we have

$$
\begin{aligned}
\left\|\Pi \delta \mathbf{q}_{i}\right\|_{\Omega_{i}} & \leq\left\|\Pi \delta \mathbf{q}_{i}-\delta \mathbf{q}_{i}\right\|_{\Omega_{i}}+\left\|\delta \mathbf{q}_{i}\right\|_{\Omega_{i}} \lesssim h^{1 / 2}\left\|\delta \mathbf{q}_{i}\right\|_{1 / 2, \Omega_{i}}+\left\|\delta \mathbf{q}_{i}\right\|_{\Omega_{i}} \\
& \lesssim \sum_{j}\left(h^{1 / 2}\left\|\mathcal{Q}_{h, i}^{R T} \mathbf{q} \cdot \mathbf{n}_{i}-\left(\mathcal{Q}_{h, 0}^{R T} \mathbf{q} \cdot \mathbf{n}\right)_{i}\right\|_{\Gamma_{i, j}}+\left\|\mathcal{Q}_{h, i}^{R T} \mathbf{q} \cdot \mathbf{n}_{i}-\left(\mathcal{Q}_{h, 0}^{R T} \mathbf{q} \cdot \mathbf{n}\right)_{i}\right\|_{-1 / 2, \Gamma_{i, j}}\right)
\end{aligned}
$$

where we have used an estimate for any divergence-free vector $\boldsymbol{\psi}[45]$

$$
\|\Pi \boldsymbol{\psi}-\boldsymbol{\psi}\|_{\Omega_{i}} \lesssim h^{r}\|\boldsymbol{\psi}\|_{r, \Omega_{i}}, 0<r \leq 1
$$

Note that the above result was shown in [45] for the Raviart-Thomas spaces, but it can be easily extended to the other mixed finite element spaces under consideration. Applying Lemma 5.9 and the trace theorem (see Thm. 1.5.2.1 in [35]):

$$
\|q\|_{r, \Gamma_{i, j}} \lesssim\|q\|_{r+1 / 2, \Omega_{i}}, \quad r>0
$$

(5.31) yields the following result.

Lemma 5.10. Under assumption (5.18), there exists a projection operator $\Pi_{0}:\left(H^{1 / 2+\epsilon}(\Omega)\right)^{d} \cap \mathbf{V} \rightarrow \mathbf{V}_{h, 0}$ such that

$$
\begin{aligned}
& \left(\nabla \cdot\left(\Pi_{0} \mathbf{q}-\mathbf{q}\right), w\right)_{\Omega_{i}}=0, \quad w \in W_{h}, \quad 1 \leq i \leq n, \\
& \left\|\Pi_{0} \mathbf{q}-\Pi \mathbf{q}\right\| \lesssim\|\mathbf{q}\|_{r+1 / 2} h^{r} H^{1 / 2}, \quad 0<r \leq 1, \\
& \left\|\Pi_{0} \mathbf{q}-\mathbf{q}\right\| \lesssim \sum_{i=1}^{n}\|\mathbf{q}\|_{1, \Omega_{i}} h+\|\mathbf{q}\|_{r+1 / 2} h^{r} H^{1 / 2}, \quad 0<r \leq 1 .
\end{aligned}
$$

Inequality (5.36) is a direct consequence of (5.35) and (5.2).

In the next Lemma we establish some continuity properties for $\Pi_{0}$ and $\Pi_{0}^{R T}$.

Lemma 5.11. Under assumption (5.18), on simplicial, quadrilaterals, and hexahedral elements,

$$
\begin{gathered}
\left\|\Pi_{0} \mathbf{q}\right\|+\left\|\Pi_{0}^{R T} \mathbf{q}\right\| \lesssim \sum_{i=1}^{n}\|\mathbf{q}\|_{1, \Omega_{i}}+\|\mathbf{q}\|_{r+1 / 2}, \quad 0<r \leq 1, \\
\sum_{E \in \mathcal{T}_{h}}\left(\left\|\Pi_{0} \mathbf{q}\right\|_{1, E}+\left\|\Pi_{0}^{R T} \mathbf{q}\right\|_{1, E}\right) \lesssim \sum_{i=1}^{n}\|\mathbf{q}\|_{1, \Omega_{i}}+h^{r-1} H^{1 / 2}\|\mathbf{q}\|_{r+1 / 2}, \quad 0<r \leq 1, \\
\forall E \in \mathcal{T}_{h}, \quad\left\|\nabla \cdot \Pi_{0} \mathbf{q}\right\|_{E}+\left\|\nabla \cdot \Pi_{0}^{R T} \mathbf{q}\right\|_{E} \lesssim\|\nabla \cdot \mathbf{q}\|_{E} .
\end{gathered}
$$


Proof. We present the proof for $\Pi_{0}$. The proof for $\Pi_{0}^{R T}$ is similar. Bound (5.37) for $\Pi_{0}$ follows from the triangle inequality $\left\|\Pi_{0} \mathbf{q}\right\| \leq\left\|\mathbf{q}-\Pi_{0} \mathbf{q}\right\|+\|\mathbf{q}\|$ and (5.36).

For (5.38), we use the triangle and inverse inequality:

$$
\sum_{E \in \mathcal{T}_{h}}\left\|\Pi_{0} \mathbf{q}\right\|_{1, E} \lesssim \sum_{E \in \mathcal{T}_{h}}\left(\left\|\Pi_{0} \mathbf{q}-\Pi \mathbf{q}\right\|_{1, E}+\|\Pi \mathbf{q}\|_{1, E}\right) \lesssim \sum_{E \in \mathcal{T}_{h}}\left(h^{-1}\left\|\Pi_{0} \mathbf{q}-\Pi \mathbf{q}\right\|_{E}+\|\Pi \mathbf{q}\|_{1, E}\right)
$$

Bound (5.38) follows by combining the above inequality with (5.35) and (5.5).

To show (5.39), we first note that if $\mathbf{v} \in \mathbf{V}_{h}$ and $(\nabla \cdot \mathbf{v}, w)=0 \forall w \in W_{h}$, then $\nabla \cdot \mathbf{v}=0$. This follows from (3.11), and (3.10). Now the definition of $\Pi_{0}$ implies that $\nabla \cdot \Pi_{0} \mathbf{q}=\nabla \cdot \Pi \mathbf{q}$. Furthermore, using (3.13) and $(3.6)$,

$$
\|\nabla \cdot \Pi \mathbf{q}\|_{E} \lesssim h_{E}^{-d / 2}\|\hat{\nabla} \cdot \hat{\Pi} \hat{\mathbf{q}}\|_{\hat{E}} \leq h_{E}^{-d / 2}\|\hat{\nabla} \cdot \hat{\mathbf{q}}\|_{\hat{E}} \lesssim\|\nabla \cdot \mathbf{q}\|_{E}
$$

where we have used that $\hat{\nabla} \cdot \hat{\Pi} \hat{\mathbf{q}}$ is the $L^{2}(\hat{E})$-projection of $\hat{\nabla} \cdot \hat{\mathbf{q}}$. This completes the proof of (5.39).

In the convergence analysis below we will need the trace inequality (see [33])

$$
\langle q, \mathbf{v} \cdot \mathbf{n}\rangle_{\partial \Omega_{i}} \lesssim\|q\|_{1 / 2, \partial \Omega_{i}}\|\mathbf{v}\|_{H\left(\operatorname{div} ; \Omega_{i}\right)}
$$

and the following lemma.

Lemma 5.12. For all $\mathbf{v} \in \mathbf{V}_{h, i}^{R T}$,

$$
\left\|\mathbf{v} \cdot \mathbf{n}_{i}\right\|_{\partial E} \approx h^{-1 / 2}\|\mathbf{v}\|_{E} .
$$

Proof. The result follows from the scaling estimate [56]: $\|\mathbf{v}\|_{E} \approx h^{(2-d) / 2}\|\hat{\mathbf{v}}\|_{\hat{E}}$, the norm equivalence on the reference element $\hat{E}:\left\|\hat{\mathbf{v}} \cdot \hat{\mathbf{n}}_{i}\right\|_{\partial \hat{E}} \bar{\sim}\|\hat{\mathbf{v}}\|_{\hat{E}}$, and (3.12).

\section{Convergence Analysis}

\subsection{Solvability}

The next lemma shows the solvability of the multiscale mortar MFMFE method.

Lemma 6.1. Assume that (5.23) holds. Then, there exists a unique solution of (4.1)-(4.3).

Proof. It is sufficient to show the uniqueness since (4.1)-(4.3) is a finite-dimensional linear square system. Let $f=0$ and $g=0$. Choosing $\mathbf{v}=\mathbf{u}_{h}, w=p_{h}$, and $\mu=\lambda_{H}$, adding (4.1)-(4.3) together, and summing over $1 \leq i \leq n$ implies that

$$
\sum_{i=1}^{n}\left(K^{-1} \mathbf{u}_{h}, \mathbf{u}_{h}\right)_{Q, \Omega_{i}}=0 .
$$

The norm equivalence (3.31) implies that $\mathbf{u}_{h}=0$. Given $p_{h} \in W_{h} \subset L^{2}(\Omega)$, there exists $\mathbf{q} \in\left(H^{1}(\Omega)\right)^{d}$ such that $\nabla \cdot \mathbf{q}=p_{h}$. Such vector field can be easily constructed by solving

$$
\Delta \phi=\tilde{p}_{h} \text { in } B, \quad \phi=0 \text { on } \partial B,
$$

where $B$ is an open ball containing $\Omega$ and $\tilde{p}_{h}$ is the extension of $p_{h}$ by zero on $B \backslash \Omega$, and taking $\mathbf{q}=\nabla \phi$. By elliptic regularity [43], $\phi \in H^{2}(B)$ and therefore $\mathbf{q} \in\left(H^{1}(\Omega)\right)^{d}$, satisfying $\|\mathbf{q}\|_{1} \lesssim\left\|\tilde{p}_{h}\right\|$. The above construction is possible since no boundary conditions on $\partial \Omega$ are imposed on the velocity field. We note that a vector field $\mathbf{q}$ with the required properties can also be constructed for a Lipschitz domain $\Omega$ when flux boundary conditions are imposed on (part of) $\partial \Omega[31,33]$.

Take $\mathbf{v}=\Pi_{0} \mathbf{q}$ in (4.1) to get $0=\sum_{i=1}^{n}\left(p_{h}, \nabla \cdot \Pi_{0} \mathbf{q}\right)=\left(p_{h}, \nabla \cdot \mathbf{q}\right)=\left\|p_{h}\right\|^{2}$, implying $p_{h}=0$. Then, (4.1) gives $0=\left\langle\lambda_{H}, \Pi^{R T} \mathbf{v} \cdot \mathbf{n}_{i}\right\rangle_{\Gamma_{i}}=\left\langle\mathcal{Q}_{h, i}^{R T} \lambda_{H}, \Pi^{R T} \mathbf{v} \cdot \mathbf{n}_{i}\right\rangle_{\Gamma_{i}}$ for all $\mathbf{v} \in \mathbf{V}_{h}$. Also, there exists $\mathbf{v} \in \mathbf{V}_{h}^{R T}$ such that $\mathbf{v} \cdot \mathbf{n}_{i}=\mathcal{Q}_{h, i}^{R T} \lambda_{H}$, implying $\mathcal{Q}_{h, i}^{R T} \lambda_{H}=0$ and, due to (5.23), $\lambda_{H}=0$. 


\subsection{Optimal convergence estimate for the velocity}

In this section we derive a priori error estimates for the velocity. Subtracting (5.15)-(5.16) from (2.3)-(2.4) gives the error equations

$$
\begin{aligned}
\left(K^{-1} \mathbf{u}, \mathbf{v}\right)-\left(K^{-1} \mathbf{u}_{h}, \mathbf{v}\right)_{Q}= & \sum_{i=1}^{n}\left(p-p_{h}, \nabla \cdot \mathbf{v}\right)_{\Omega_{i}}-\sum_{i=1}^{n}\left\langle p, \mathbf{v} \cdot \mathbf{n}_{i}\right\rangle_{\Gamma_{i}} \\
& -\sum_{i=1}^{n}\left\langle g,\left(\mathbf{v}-\Pi^{R T} \mathbf{v}\right) \cdot \mathbf{n}_{i}\right\rangle_{\partial \Omega_{i} / \Gamma}, \quad \forall \mathbf{v} \in \mathbf{V}_{h, 0} \\
\sum_{i=1}^{n}\left(\nabla \cdot\left(\mathbf{u}-\mathbf{u}_{h}\right), w\right)_{\Omega_{i}}= & 0, \quad \forall w \in W_{h} .
\end{aligned}
$$

For the permeability tensor $K$, we will use the following notation. Let $W_{\mathcal{T}_{h}}^{k, \infty}$ consist of functions $\phi$ such that $\left.\phi\right|_{E} \in W^{k, \infty}(E)$ for all $E \in \mathcal{T}_{h}$ and $\|\phi\|_{k, \infty, E}$ is uniformly bounded. Let $\|\phi\|_{k, \infty}=\max _{E \in \mathcal{T}_{h}}\|\phi\|_{k, \infty, E}$.

The following two lemmas give bounds on terms that appear in the velocity error analysis.

Lemma $6.2([39,56])$. On $h^{2}$-parallelograms and $h^{2}$-parallelepipeds, if $K^{-1} \in W_{\mathcal{T}_{h}}^{1, \infty}$, then for all $\mathbf{v} \in \mathbf{V}_{h}$,

$$
\left|\left(K^{-1} \Pi \mathbf{u}, \mathbf{v}-\Pi^{R T} \mathbf{v}\right)_{Q}\right| \lesssim \sum_{i=1}^{n} h\left\|K^{-1}\right\|_{1, \infty}\|\mathbf{u}\|_{1, \Omega_{i}}\|\mathbf{v}\|_{\Omega_{i}} .
$$

Lemma $6.3([39,56])$. On simplicial elements, $h^{2}$-parallelograms, and $h^{2}$-parallelepipeds, if $K^{-1} \in W_{\mathcal{T}_{h}}^{1, \infty}$, then for all $\mathbf{q} \in \mathbf{V}_{h}$ and for all $\mathbf{v} \in \mathbf{V}_{h}^{R T}$, the numerical quadrature error satisfies

$$
\left|\sigma\left(K^{-1} \mathbf{q}, \mathbf{v}\right)\right| \lesssim \sum_{E \in \mathcal{T}_{h}} h\left\|K^{-1}\right\|_{1, \infty, E}\|\mathbf{q}\|_{1, E}\|\mathbf{v}\|_{0, E}
$$

Furthermore, on simplicial elements, the above inequality also holds for all $\mathbf{q}, \mathbf{v} \in \mathbf{V}_{h}$.

We are now ready to establish the main a priori velocity bound.

Theorem 6.4. Let $K^{-1} \in W_{\mathcal{T}_{h}}^{1, \infty}$. For the velocity $\mathbf{u}_{h}$ of the mortar MFMFE method (4.1)-(4.3) on simplicial elements, $h^{2}$-parallelograms, and $h^{2}$-parallelepipeds, if (5.18) holds, then

$$
\begin{aligned}
\sum_{i=1}^{n}\left\|\nabla \cdot\left(\mathbf{u}-\mathbf{u}_{h}\right)\right\|_{\Omega_{i}} & \lesssim \sum_{i=1}^{n} h\|\nabla \cdot \mathbf{u}\|_{1, \Omega_{i}}, \\
\left\|\mathbf{u}-\mathbf{u}_{h}\right\| & \lesssim \sum_{i=1}^{n}\left(H^{s-1 / 2}\|p\|_{s+1 / 2, \Omega_{i}}+h\|\mathbf{u}\|_{1, \Omega_{i}}\right)+h^{r} H^{1 / 2}\|\mathbf{u}\|_{r+1 / 2},
\end{aligned}
$$

where $1 / 2 \leq s \leq m+1,0<r \leq 1$.

Proof. First we note that

$$
\nabla \cdot\left(\Pi \mathbf{u}-\mathbf{u}_{h}\right)=0 \quad \text { and } \quad \nabla \cdot\left(\Pi_{0} \mathbf{u}-\mathbf{u}_{h}\right)=0 \text { in } \Omega_{i}, i=1, \ldots, n,
$$

which follow from (6.3), (3.17), (5.34), (3.11), and (3.10). The divergence error bound (6.6) now follows from (5.3).

For all $\mathbf{v} \in \mathbf{V}_{h, 0}$ such that $\nabla \cdot \mathbf{v}=0$ in each subdomain, the error equation (6.2) gives

$$
\left(K^{-1} \mathbf{u}, \mathbf{v}\right)-\left(K^{-1} \mathbf{u}_{h}, \mathbf{v}\right)_{Q}=-\sum_{i=1}^{n}\left\langle p,\left(\mathbf{v}-\Pi^{R T} \mathbf{v}\right) \cdot \mathbf{n}_{i}\right\rangle_{\Gamma_{i}}-\sum_{i=1}^{n}\left\langle p-\mathcal{I}_{H}^{c} p, \Pi^{R T} \mathbf{v} \cdot \mathbf{n}_{i}\right\rangle_{\Gamma_{i}}-\sum_{i=1}^{n}\left\langle g,\left(\mathbf{v}-\Pi^{R T} \mathbf{v}\right) \cdot \mathbf{n}_{i}\right\rangle_{\partial \Omega_{i} / \Gamma},
$$


where we have used that

$$
\sum_{i=1}^{n}\left\langle\mathcal{I}_{H}^{c} p, \Pi^{R T} \mathbf{v} \cdot \mathbf{n}_{i}\right\rangle_{\Gamma_{i}}=0
$$

Taking $\mathbf{v}-\Pi^{R T} \mathbf{v} \in \mathbf{V}_{h}$ as a test function in (2.3) gives

$$
\left(K^{-1} \mathbf{u}, \mathbf{v}-\Pi^{R T} \mathbf{v}\right)=-\sum_{i=1}^{n}\left\langle g,\left(\mathbf{v}-\Pi^{R T} \mathbf{v}\right) \cdot \mathbf{n}_{i}\right\rangle_{\partial \Omega_{i} / \Gamma}-\sum_{i=1}^{n}\left\langle p,\left(\mathbf{v}-\Pi^{R T} \mathbf{v}\right) \cdot \mathbf{n}_{i}\right\rangle_{\Gamma_{i}} .
$$

The above two equations imply

$$
\left(K^{-1} \mathbf{u}_{h}, \mathbf{v}\right)_{Q}=\left(K^{-1} \mathbf{u}, \Pi^{R T} \mathbf{v}\right)+\sum_{i=1}^{n}\left\langle p-\mathcal{I}_{H}^{c} p, \Pi^{R T} \mathbf{v} \cdot \mathbf{n}_{i}\right\rangle_{\Gamma_{i}} \quad \forall \mathbf{v} \in \mathbf{V}_{h, 0} \text { s.t. } \nabla \cdot \mathbf{v}=0 .
$$

By using (6.10), we rewrite $\left(K^{-1}\left(\Pi_{0} \mathbf{u}-\mathbf{u}_{h}\right), \mathbf{v}\right)_{Q}$ as

$$
\begin{aligned}
\left(K^{-1}\left(\Pi_{0} \mathbf{u}-\mathbf{u}_{h}\right), \mathbf{v}\right)_{Q}= & \left(K^{-1} \Pi_{0} \mathbf{u}, \mathbf{v}\right)_{Q}-\left(K^{-1} \mathbf{u}, \Pi^{R T} \mathbf{v}\right)-\sum_{i=1}^{n}\left\langle p-\mathcal{I}_{H}^{c} p, \Pi^{R T} \mathbf{v} \cdot \mathbf{n}_{i}\right\rangle_{\Gamma_{i}} \\
= & \left(K^{-1}\left(\Pi_{0} \mathbf{u}-\Pi \mathbf{u}\right), \mathbf{v}\right)_{Q}+\left(K^{-1} \Pi \mathbf{u}, \mathbf{v}-\Pi^{R T} \mathbf{v}\right)_{Q}-\sigma\left(K^{-1} \Pi \mathbf{u}, \Pi^{R T} \mathbf{v}\right) \\
& +\left(K^{-1}(\Pi \mathbf{u}-\mathbf{u}), \Pi^{R T} \mathbf{v}\right)-\sum_{i=1}^{n}\left\langle p-\mathcal{I}_{H}^{c} p, \Pi^{R T} \mathbf{v} \cdot \mathbf{n}_{i}\right\rangle_{\Gamma_{i}} .
\end{aligned}
$$

The first and fourth terms on the right hand side of (6.11) can be estimated by using (5.35) and (5.2), respectively:

$$
\begin{aligned}
\left(K^{-1}\left(\Pi_{0} \mathbf{u}-\Pi \mathbf{u}\right), \mathbf{v}\right)_{Q} & \lesssim h^{r} H^{1 / 2}\|\mathbf{u}\|_{r+1 / 2}\|\mathbf{v}\|, \quad 0<r \leq 1 \\
\left(K^{-1}(\Pi \mathbf{u}-\mathbf{u}), \Pi^{R T} \mathbf{v}\right) & \lesssim \sum_{i=1}^{n} h\|\mathbf{u}\|_{1, \Omega_{i}}\|\mathbf{v}\|_{\Omega_{i}}
\end{aligned}
$$

where we have also used (3.24). Using (6.5), (3.24), and (5.5), we bound the third term on the right in (6.11) as

$$
\left|\sigma\left(K^{-1} \Pi \mathbf{u}, \Pi^{R T} \mathbf{v}\right)\right| \lesssim \sum_{E \in \mathcal{T}_{h}} h\|\Pi \mathbf{u}\|_{1, E}\left\|\Pi^{R T} \mathbf{v}\right\|_{E} \lesssim \sum_{i=1}^{n} h\|\mathbf{u}\|_{1, \Omega_{i}}\|\mathbf{v}\|_{\Omega_{i}} .
$$

The last term on the right hand side of (6.11) can be estimated from (5.40), (5.11), (5.33), and (3.24) as

$$
\sum_{i=1}^{n}\left\langle\mathcal{I}_{H}^{c} p-p, \Pi \Pi^{R T} \mathbf{v} \cdot \mathbf{n}_{i}\right\rangle_{\Gamma_{i}} \leq \sum_{i=1}^{n}\left\|\mathcal{I}_{H}^{c} p-p\right\|_{1 / 2, \partial \Omega_{i}}\left\|\Pi^{R T} \mathbf{v}\right\|_{H\left(\operatorname{div} ; \Omega_{i}\right)} \lesssim \sum_{i=1}^{n}\|p\|_{s+1 / 2, \Omega_{i}} H^{s-1 / 2}\|\mathbf{v}\|_{\Omega_{i}}
$$

for $1 / 2 \leq s \leq m+1$, where we have also used that $\nabla \cdot \Pi^{R T} \mathbf{v}=0$.

Now let $\mathbf{v}=\Pi_{0} \mathbf{u}-\mathbf{u}_{h}$. A combination of (6.11)-(6.15), (3.31), Lemma 6.2, and (5.36) completes the proof of (6.7).

\subsection{Superconvergence estimates for the velocity}

In this section we establish velocity superconvergence on rectangular and cuboid grids in the case of diagonal permeability tensor. We note that velocity superconvergence is observed numerically on $h^{2}$-parallelograms and $h^{2}$-parallelepipeds. The proof is based on combining the super-closeness between $\Pi_{0}^{R T}$ and $\Pi^{R T}(5.20)$, superconvergence for $\Pi^{R T}$ on a single domain, and superconvergence for the quadrature error $\sigma(\cdot, \cdot)$. The last bound requires restricting the integrands to $\mathrm{RT}_{0}$, which necessitates modifying the argument from the previous section. We make use of the following superconvergence results. 
Lemma 6.5 ([29], Thm. 5.1). If $\mathbf{u} \in H^{2}\left(\Omega_{i}\right), 1 \leq i \leq n, K^{-1} \in W_{\mathcal{T}_{h}}^{1, \infty}(\Omega)$, and $\mathbf{v} \in \mathbf{V}_{h}^{R T}$, then

$$
\left(K^{-1}\left(\Pi^{R T} \mathbf{u}-\mathbf{u}\right), \mathbf{v}\right) \lesssim \sum_{i=1}^{n} h^{t+1}\left\|K^{-1}\right\|_{1, \infty}\left(\|\mathbf{u}\|_{t+1, \Omega_{i}}\|\mathbf{v}\|_{\Omega_{i}}+\|\mathbf{u}\|_{1, \Omega_{i}}\|\nabla \cdot \mathbf{v}\|_{\Omega_{i}}\right), \quad 0 \leq t \leq 1 .
$$

Lemma 6.6 ([17], Lem. 4.3). If $\mathbf{u} \in H^{2}\left(\Omega_{i}\right), 1 \leq i \leq n, K^{-1} \in W_{\mathcal{T}_{h}}^{2, \infty}(\Omega)$, and $\mathbf{v} \in \mathbf{V}_{h}^{R T}$, then

$$
\left|\sigma\left(K^{-1} \Pi^{R T} \mathbf{u}, \mathbf{v}\right)\right| \lesssim \sum_{i=1}^{n} h^{t+1}\left\|K^{-1}\right\|_{2, \infty}\left(\|\mathbf{u}\|_{t+1, \Omega_{i}}\|\mathbf{v}\|_{\Omega_{i}}+\|\mathbf{u}\|_{1, \Omega_{i}}\|\nabla \cdot \mathbf{v}\|_{\Omega_{i}}\right), \quad 0 \leq t \leq 1 / 2 .
$$

We note that both results above are established in two dimensions for $h^{2}$-parallelograms and a full tensor with Neumann boundary conditions. Here we use them for a diagonal tensor with Dirichlet boundary conditions on rectangular and cuboid grids. The extensions to three dimensions are straightforward.

Next, we establish a bound on a term that appears in the proof of Theorem 6.8. This is the term that limits the proof to rectangular-type grids.

Lemma 6.7. Assume that $K$ is a diagonal tensor and $K^{-1} \in W_{\mathcal{T}_{h}}^{1, \infty}$. Then for all $\mathbf{q} \in \mathbf{V}_{h}$ and $\mathbf{v} \in \mathbf{V}_{h}^{R T}$ on rectangular and cuboid grids,

$$
\left|\left(K^{-1}\left(\mathbf{q}-\Pi^{R T} \mathbf{q}\right), \mathbf{v}\right)_{Q}\right| \lesssim \sum_{i=1}^{n} h\left\|K^{-1}\right\|_{1, \infty}\left\|\mathbf{q}-\Pi^{R T} \mathbf{q}\right\|_{\Omega_{i}}\|\mathbf{v}\|_{\Omega_{i}} .
$$

Proof. Let $\overline{K^{-1}}$ denote the $L^{2}$-projection of $K^{-1}$ onto the space of piecewise constant tensors. On any element $E$,

$$
\left(K^{-1}\left(\mathbf{q}-\Pi^{R T} \mathbf{q}\right), \mathbf{v}\right)_{Q, E}=\left(\left(K^{-1}-\overline{K^{-1}}\right)\left(\mathbf{q}-\Pi^{R T} \mathbf{q}\right), \mathbf{v}\right)_{Q, E}+\left(\overline{K^{-1}}\left(\mathbf{q}-\Pi^{R T} \mathbf{q}\right), \mathbf{v}\right)_{Q, E} .
$$

Using (3.31), the first term on the right-side of (6.19) can be bounded as

$$
\left(\left(K^{-1}-\overline{K^{-1}}\right)\left(\mathbf{q}-\Pi^{R T} \mathbf{q}\right), \mathbf{v}\right)_{Q, E} \lesssim h\left|K^{-1}\right|_{1, \infty, E}\left\|\mathbf{q}-\Pi^{R T} \mathbf{q}\right\|_{E}\|\mathbf{v}\|_{E} .
$$

Next, consider the second term on the right-hand side of (6.19). The proofs for 2D and 3D elements are very similar. For simplicity, we give a proof for the $2 \mathrm{D}$ case. Let $\mathbf{z} \equiv \mathbf{q}-\Pi^{R T} \mathbf{q}$. Let $\mathbf{z}=\left[z_{1}, z_{2}\right]^{T}$ and $\mathbf{v}=\left[v_{1}, v_{2}\right]$. Since the grid is orthogonal, $J_{E}$ is constant and $J_{E} \overline{K^{-1}}$ is a diagonal constant tensor, $J_{E} \overline{K^{-1}}=\operatorname{diag}\left(k_{1}, k_{2}\right)$. Denote the vertices of $E$ by $\mathbf{r}_{1}, \ldots, \mathbf{r}_{4}$, where $\mathbf{r}_{1}$ is the lower left vertex and the other vertices are numbered in a counter clockwise direction. By the definition of the quadrature rule (3.30),

$$
\left(\overline{K^{-1}}\left(\mathbf{q}-\Pi^{R T} \mathbf{q}\right), \mathbf{v}\right)_{Q, E}=k_{1}\left(z_{1}, v_{1}\right)_{Q, E}+k_{2}\left(z_{2}, v_{2}\right)_{Q, E}
$$

and

$$
\left(z_{1}, v_{1}\right)_{Q, E}=\frac{1}{4} \sum_{i=1}^{4} z_{1}\left(\mathbf{r}_{i}\right) v_{1}\left(\mathbf{r}_{i}\right)=\frac{1}{4} v_{1}\left(\mathbf{r}_{2}\right)\left(z_{1}\left(\mathbf{r}_{2}\right)+z_{1}\left(\mathbf{r}_{3}\right)\right)+\frac{1}{4} v_{1}\left(\mathbf{r}_{4}\right)\left(z_{1}\left(\mathbf{r}_{4}\right)+z_{1}\left(\mathbf{r}_{1}\right)\right),
$$

where we used that $v_{1}\left(\mathbf{r}_{2}\right)=v_{1}\left(\mathbf{r}_{3}\right)$ and $v_{1}\left(\mathbf{r}_{4}\right)=v_{1}\left(\mathbf{r}_{1}\right)$ since $\mathbf{v} \in \mathrm{RT}_{0}(E)$. Since $\mathbf{q} \in \mathrm{BDM}_{1}(E)$, by the definition of $\Pi^{R T}$ we have $z_{1}\left(\mathbf{r}_{2}\right)+z_{1}\left(\mathbf{r}_{3}\right)=0$ and $z_{1}\left(\mathbf{r}_{4}\right)+z_{1}\left(\mathbf{r}_{1}\right)=0$. This gives $\left(z_{1}, v_{1}\right)_{Q, E}=0$, and similarly we get $\left(z_{2}, v_{2}\right)_{Q, E}=0$. Therefore

$$
\left.\overline{K^{-1}}\left(\mathbf{q}-\Pi^{R T} \mathbf{q}\right), \mathbf{v}\right)_{Q, E}=0
$$

which, combined with (6.19) and (6.20), implies the assertion of the lemma.

The following is the main result of this section. 
Theorem 6.8. Assume that the tensor $K$ is diagonal and $K^{-1} \in W_{\mathcal{T}_{h}}^{2, \infty}$. Then, if (5.18) holds, the velocity $\mathbf{u}_{h}$ of the mortar MFMFE method (4.1)-(4.3) on rectangular and cuboid grids satisfies

$$
\left\|\Pi^{R T} \mathbf{u}-\Pi^{R T} \mathbf{u}_{h}\right\| \lesssim \sum_{i=1}^{n}\left(H^{s-1 / 2}\|p\|_{s+1 / 2, \Omega_{i}}+h^{t+1}\|\mathbf{u}\|_{t+1, \Omega_{i}}\right)+h^{r} H^{1 / 2}\|\mathbf{u}\|_{r+1 / 2}
$$

where $1 / 2 \leq s<m+1,0<r \leq 1$, and $0 \leq t \leq 1 / 2$.

Proof. By the triangle inequality

$$
\left\|\Pi^{R T} \mathbf{u}-\Pi^{R T} \mathbf{u}_{h}\right\| \leq\left\|\Pi^{R T} \mathbf{u}-\Pi_{0}^{R T} \mathbf{u}\right\|+\left\|\Pi_{0}^{R T} \mathbf{u}-\Pi^{R T} \mathbf{u}_{h}\right\| .
$$

The first term on the right above is bounded in (5.20). For the second term on the right in (6.22), using the norm equivalence (3.31), we have

$$
\begin{aligned}
\left\|\Pi_{0}^{R T} \mathbf{u}-\Pi^{R T} \mathbf{u}_{h}\right\|^{2} & \lesssim\left(K^{-1}\left(\Pi_{0}^{R T} \mathbf{u}-\Pi^{R T} \mathbf{u}_{h}\right), \Pi_{0}^{R T} \mathbf{u}-\Pi^{R T} \mathbf{u}_{h}\right)_{Q} \\
& =\left(K^{-1}\left(\Pi_{0}^{R T} \mathbf{u}-\mathbf{u}_{h}\right), \Pi_{0}^{R T} \mathbf{u}-\Pi^{R T} \mathbf{u}_{h}\right)_{Q}+\left(K^{-1}\left(\mathbf{u}_{h}-\Pi^{R T} \mathbf{u}_{h}\right), \Pi_{0}^{R T} \mathbf{u}-\Pi^{R T} \mathbf{u}_{h}\right)_{Q}
\end{aligned}
$$

By (6.18), the second term on the right in (6.23) can be estimated as

$$
\left(K^{-1}\left(\mathbf{u}_{h}-\Pi^{R T} \mathbf{u}_{h}\right), \Pi_{0}^{R T} \mathbf{u}-\Pi^{R T} \mathbf{u}_{h}\right)_{Q} \lesssim \sum_{i=1}^{n} h\left\|K^{-1}\right\|_{1, \infty}\left\|\mathbf{u}_{h}-\Pi^{R T} \mathbf{u}_{h}\right\|_{\Omega_{i}}\left\|\Pi_{0}^{R T} \mathbf{u}-\Pi^{R T} \mathbf{u}_{h}\right\|_{\Omega_{i}} .
$$

Using triangle inequality and (3.25), we have

$$
\begin{aligned}
\left\|\mathbf{u}_{h}-\Pi^{R T} \mathbf{u}_{h}\right\|_{\Omega_{i}} & \leq\left\|\mathbf{u}-\mathbf{u}_{h}\right\|_{\Omega_{i}}+\left\|\mathbf{u}-\Pi^{R T} \mathbf{u}\right\|_{\Omega_{i}}+\left\|\Pi^{R T} \Pi\left(\mathbf{u}-\mathbf{u}_{h}\right)\right\|_{\Omega_{i}} \\
& \lesssim\left\|\mathbf{u}-\mathbf{u}_{h}\right\|_{\Omega_{i}}+\left\|\mathbf{u}-\Pi^{R T} \mathbf{u}\right\|_{\Omega_{i}}+\left\|\Pi \mathbf{u}-\mathbf{u}_{h}\right\|_{\Omega_{i}},
\end{aligned}
$$

where we have used (3.24). Combining (6.24) and (6.25),

$$
\begin{aligned}
& \left(K^{-1}\left(\mathbf{u}_{h}-\Pi^{R T} \mathbf{u}_{h}\right), \Pi_{0}^{R T} \mathbf{u}-\Pi^{R T} \mathbf{u}_{h}\right)_{Q} \\
& \quad \lesssim \sum_{i=1}^{n} h\left\|K^{-1}\right\|_{1, \infty}\left(\left\|\mathbf{u}-\mathbf{u}_{h}\right\|_{\Omega_{i}}+\left\|\mathbf{u}-\Pi^{R T} \mathbf{u}\right\|_{\Omega_{i}}+\left\|\Pi \mathbf{u}-\mathbf{u}_{h}\right\|_{\Omega_{i}}\right)\left\|\Pi_{0}^{R T} \mathbf{u}-\Pi^{R T} \mathbf{u}_{h}\right\|_{\Omega_{i}} .
\end{aligned}
$$

It remains to bound the first term on the right in (6.23). Using (6.10), similar to (6.11), we obtain

$$
\begin{aligned}
\left(K^{-1}\left(\Pi_{0}^{R T} \mathbf{u}-\mathbf{u}_{h}\right), \Pi_{0}^{R T} \mathbf{u}-\Pi^{R T} \mathbf{u}_{h}\right)_{Q} & \\
= & \left(K^{-1}\left(\Pi_{0}^{R T} \mathbf{u}-\Pi^{R T} \mathbf{u}\right), \Pi_{0}^{R T} \mathbf{u}-\Pi^{R T} \mathbf{u}_{h}\right)_{Q}+\left(K^{-1}\left(\Pi^{R T} \mathbf{u}-\mathbf{u}\right), \Pi_{0}^{R T} \mathbf{u}-\Pi^{R T} \mathbf{u}_{h}\right) \\
& -\sigma\left(K^{-1} \Pi^{R T} \mathbf{u}, \Pi_{0}^{R T} \mathbf{u}-\Pi^{R T} \mathbf{u}_{h}\right)-\sum_{i=1}^{n}\left\langle p-\mathcal{I}_{H}^{c} p,\left(\Pi_{0}^{R T} \mathbf{u}-\Pi^{R T} \mathbf{u}_{h}\right) \cdot \mathbf{n}_{i}\right\rangle_{\Gamma_{i}} .
\end{aligned}
$$

The first term on the right above can be bounded as

$$
\left(K^{-1}\left(\Pi_{0}^{R T} \mathbf{u}-\Pi^{R T} \mathbf{u}\right), \Pi_{0}^{R T} \mathbf{u}-\Pi^{R T} \mathbf{u}_{h}\right)_{Q} \lesssim\left\|\Pi_{0}^{R T} \mathbf{u}-\Pi^{R T} \mathbf{u}\right\|\left\|\Pi_{0}^{R T} \mathbf{u}-\Pi^{R T} \mathbf{u}_{h}\right\| .
$$

The second term on the right in (6.27) can be bounded using (6.16): for $0 \leq t \leq 1$,

$$
\left(K^{-1}\left(\Pi^{R T} \mathbf{u}-\mathbf{u}\right), \Pi_{0}^{R T} \mathbf{u}-\Pi^{R T} \mathbf{u}_{h}\right) \lesssim \sum_{i=1}^{n} h^{t+1}\left\|K^{-1}\right\|_{1, \infty}\|\mathbf{u}\|_{t+1, \Omega_{i}}\left\|\Pi_{0}^{R T} \mathbf{u}-\Pi^{R T} \mathbf{u}_{h}\right\|_{\Omega_{i}},
$$


where we have used that $\nabla \cdot\left(\Pi_{0}^{R T} \mathbf{u}-\Pi^{R T} \mathbf{u}_{h}\right)=0$. The third term on the right in (6.27) can be bounded using (6.17): for $0 \leq t \leq 1 / 2$,

$$
\sigma\left(K^{-1} \Pi^{R T} \mathbf{u}, \Pi_{0}^{R T} \mathbf{u}-\Pi^{R T} \mathbf{u}_{h}\right) \lesssim \sum_{i=1}^{n} h^{t+1}\left\|K^{-1}\right\|_{2, \infty}\|\mathbf{u}\|_{t+1, \Omega_{i}}\left\|\Pi_{0}^{R T} \mathbf{u}-\Pi^{R T} \mathbf{u}_{h}\right\|_{\Omega_{i}} .
$$

Similar to (6.15), the last term on the right in (6.27) can be estimated as, for $0<s \leq m+1$,

$$
\sum_{i=1}^{n}\left\langle p-\mathcal{I}_{H}^{c} p,\left(\Pi_{0}^{R T} \mathbf{u}-\Pi^{R T} \mathbf{u}_{h}\right) \cdot \mathbf{n}_{i}\right\rangle_{\Gamma_{i}} \lesssim \sum_{i=1}^{n} H^{s-1 / 2}\|p\|_{s+1 / 2, \Omega_{i}}\left\|\Pi_{0}^{R T} \mathbf{u}-\Pi^{R T} \mathbf{u}_{h}\right\|_{\Omega_{i}}
$$

A combination of (6.22)-(6.31) with (5.20), (6.7), and (5.2) implies (6.21).

For a scalar function $\phi\left(x_{1}, \ldots, x_{d}\right)$ in a rectangular or cuboid element $E$, let $\|\phi\|_{i, E}$ denote an approximation integral of $|\phi|^{2}$ using exact integration rule in $x_{i}$ and midpoint rule in the other directions. Then, for $\mathbf{q}=$ $\left(q_{1}, \ldots, q_{d}\right)^{T}$, let

$$
\|\mathbf{q}\|^{2}=\sum_{E \in \mathcal{T}_{h}} \sum_{i=1}^{d}\left\|q_{i}\right\|_{i, E}^{2},
$$

and note that $\left\|\mathbf{v}_{h}\right\|=\left\|\mathbf{v}_{h}\right\|$ if $\mathbf{v}_{h} \in \mathbf{V}_{h}^{R T}$ on rectangular or cuboid grids. The reader is cautioned not to confuse the above norm with the norm $\|\cdot\|_{k, \infty}$ used for the permeability tensor $K$.

Theorem 6.9. Assume that the tensor $K$ is diagonal and $K^{-1} \in W_{\mathcal{T}_{h}}^{2, \infty}$. Then, if (5.18) holds, the velocity $\mathbf{u}_{h}$ of the mortar MFMFE method (4.1)-(4.3) on rectangular and cuboid grids satisfies

$$
\left.\left\|\mathbf{u}-\Pi^{R T} \mathbf{u}_{h}\right\| \lesssim \sum_{i=1}^{n}\left(H^{s-1 / 2}\|p\|_{s+1 / 2, \Omega_{i}}+h^{t+1}\|\mathbf{u}\|_{t+1, \Omega_{i}}\right)+h^{r} H^{1 / 2}\|\mathbf{u}\|_{r+1 / 2}\right),
$$

where $1 / 2 \leq s<m+1,0<r \leq 1$, and $0 \leq t \leq 1 / 2$.

Proof. It is well known [26] that the usual RT interpolant $\Pi^{R T}$ exhibits superconvergence:

$$
\left\|\mathbf{u}-\Pi^{R T} \mathbf{u}\right\|_{\Omega_{i}} \lesssim h^{t+1}\|\mathbf{u}\|_{t+1, \Omega_{i}}, \quad 0 \leq t \leq 1 .
$$

By the triangle inequality

$$
\left\|\mathbf{u}-\Pi^{R T} \mathbf{u}_{h}\right\| \leq\left\|\mathbf{u}-\Pi^{R T} \mathbf{u}\right\|+\left\|\Pi^{R T} \mathbf{u}-\Pi^{R T} \mathbf{u}_{h}\right\|=\left\|\mathbf{u}-\Pi^{R T} \mathbf{u}\right\|+\left\|\Pi^{R T} \mathbf{u}-\Pi^{R T} \mathbf{u}_{h}\right\| .
$$

A combination of the above estimate, (6.34), and (6.21) completes the proof.

\subsection{Optimal convergence estimate for the pressure}

In this section we prove an inf-sup condition and establish an optimal convergence for the pressure.

Lemma 6.10. Spaces $\mathbf{V}_{h, 0}^{R T} \times W_{h}$ satisfy the inf-sup condition: for all $w \in W_{h}$,

$$
\sup _{0 \neq \mathbf{v} \in \mathbf{V}_{h, 0}^{R T}} \frac{\sum_{i=1}^{n}(\nabla \cdot \mathbf{v}, w)_{\Omega_{i}}}{\sum_{i=1}^{n}\|\mathbf{v}\|_{H\left(\operatorname{div} ; \Omega_{\mathrm{i}}\right)}} \gtrsim\|w\|
$$


Proof. Let $w \in W_{h}$. It is enough to show that there exists $\mathbf{v} \in \mathbf{V}_{h, 0}^{R T}$ such that

$$
\sum_{i=1}^{n}(\nabla \cdot \mathbf{v}, w)_{\Omega_{i}}=\|w\|^{2} \text {, and } \sum_{i=1}^{n}\|\mathbf{v}\|_{H\left(\operatorname{div} ; \Omega_{i}\right)} \lesssim\|w\| .
$$

As in the proof of Lemma 6.1, see (6.1), there exists $\mathbf{q} \in\left(H^{1}(\Omega)\right)^{d}$ such that

$$
\nabla \cdot \mathbf{q}=w \text { and } \quad\|\mathbf{q}\|_{1} \lesssim\|w\| .
$$

It is easy to see that $\mathbf{v}=\Pi_{0}^{R T} \mathbf{q}$ satisfies (6.36), using (6.37), (5.19), (5.37) and (5.39).

Theorem 6.11. Let $K^{-1} \in W^{1, \infty}\left(\Omega_{i}\right), 1 \leq i \leq n$. For the pressure $p_{h}$ of the mortar MFMFE method (4.1)(4.3) on simplicial elements, $h^{2}$-parallelograms, and $h^{2}$-parallelepipeds, if (5.18) holds, then

$$
\left\|p-p_{h}\right\| \lesssim \sum_{i=1}^{n}\left(h\|p\|_{1, \Omega_{i}}+h\|\mathbf{u}\|_{1, \Omega_{i}}+H^{s-1 / 2}\|p\|_{s+1 / 2, \Omega_{i}}\right)+h^{r} H^{1 / 2}\|\mathbf{u}\|_{r+1 / 2},
$$

where $1 / 2 \leq s \leq m+1$ and $0<r \leq 1$.

Proof. Using (6.35),

$$
\begin{aligned}
\left\|Q_{h} p-p_{h}\right\| & \lesssim \sup _{\substack{0 \neq \mathbf{v} \in \mathbf{V}_{h, 0}^{R T}\\
}} \frac{\sum_{i=1}^{n}\left(\nabla \cdot \mathbf{v}, Q_{h} p-p_{h}\right)_{\Omega_{i}}}{\sum_{i=1}^{n}\|\mathbf{v}\|_{H\left(\operatorname{div} ; \Omega_{i}\right)}} \\
& =\sup _{0 \neq \mathbf{v} \in \mathbf{V}_{h, 0}^{R T}} \frac{\left(K^{-1} \mathbf{u}, \mathbf{v}\right)-\left(K^{-1} \mathbf{u}_{h}, \mathbf{v}\right)_{Q}+\sum_{i=1}^{n}\left\langle p-\mathcal{I}_{H}^{c} p, \mathbf{v} \cdot \mathbf{n}_{i}\right\rangle_{\Gamma_{i}}}{\sum_{i=1}^{n}\|\mathbf{v}\|_{H\left(\operatorname{div} ; \Omega_{i}\right)}},
\end{aligned}
$$

where we have used (6.2), the fact that $\mathbf{V}_{h, 0}^{R T} \subset \mathbf{V}_{h, 0}$, (5.7), and (6.9). We reformulate the first two terms and the last term in the numerator as

$$
\left(K^{-1} \mathbf{u}, \mathbf{v}\right)-\left(K^{-1} \mathbf{u}_{h}, \mathbf{v}\right)_{Q}=\left(K^{-1}(\mathbf{u}-\Pi \mathbf{u}), \mathbf{v}\right)-\left(K^{-1}\left(\mathbf{u}_{h}-\Pi \mathbf{u}\right), \mathbf{v}\right)_{Q}+\sigma\left(K^{-1} \Pi \mathbf{u}, \mathbf{v}\right) .
$$

By (5.40) and (5.11), the last term in the numerator of (6.39) can be estimated as

$$
\sum_{i=1}^{n}\left\langle p-\mathcal{I}_{H}^{c} p, \mathbf{v} \cdot \mathbf{n}_{i}\right\rangle_{\Gamma_{i}} \lesssim \sum_{i=1}^{n} H^{s-1 / 2}\|p\|_{s+1 / 2, \Omega_{i}}\|\mathbf{v}\|_{H\left(\operatorname{div} ; \Omega_{i}\right)}, \quad 1 / 2 \leq s \leq m+1
$$

A combination of (6.39)-(6.41), (6.5), (5.5), (5.2), (3.31), and (6.7) implies (6.38).

\subsection{Superconvergence for the pressure}

In this section we employ a duality argument to obtain a superconvergence for the pressure at the element centers of mass. We begin with some auxiliary lemmas needed in the analysis.

Lemma 6.12. For all $\mathbf{q} \in H^{1}(\Omega)^{d}$,

$$
\left\|\Pi^{R T} \Pi_{0} \mathbf{q}-\mathbf{q}\right\| \lesssim \sum_{i=1}^{n} h\|\mathbf{q}\|_{1, \Omega_{i}}+h^{r} H^{1 / 2}\|\mathbf{q}\|_{r+1 / 2}, \quad 0<r \leq 1 .
$$


Proof. Using (5.2) and (5.36), we have

$$
\begin{aligned}
\left\|\Pi^{R T} \Pi_{0} \mathbf{q}-\mathbf{q}\right\| & \leq\left\|\Pi^{R T} \Pi_{0} \mathbf{q}-\Pi_{0} \mathbf{q}\right\|+\left\|\Pi_{0} \mathbf{q}-\mathbf{q}\right\| \lesssim \sum_{E \in \mathcal{T}_{h}} h\left\|\Pi_{0} \mathbf{q}\right\|_{1, E}+\sum_{i=1}^{n} h\|\mathbf{q}\|_{1, \Omega_{i}}+h^{r} H^{1 / 2}\|\mathbf{q}\|_{r+1 / 2} \\
& \lesssim \sum_{i=1}^{n} h\|\mathbf{q}\|_{1, \Omega_{i}}+h^{r} H^{1 / 2}\|\mathbf{q}\|_{r+1 / 2}, \quad 0<r \leq 1
\end{aligned}
$$

where we have used (5.38) in the last inequality.

Lemma 6.13 $([39,56])$. Let $K^{-1} \in W_{\mathcal{T}_{h}}^{2, \infty}$. On simplicial elements, $h^{2}$-parallelograms, and regular $h^{2}$ parallelepipeds, for all $\mathbf{q} \in \mathbf{V}_{h}, \mathbf{v} \in \mathbf{V}_{h}^{R T}$,

$$
\left|\sigma\left(K^{-1} \mathbf{q}, \mathbf{v}\right)\right| \lesssim \sum_{E \in \mathcal{T}_{h}} h^{2}\left\|K^{-1}\right\|_{2, \infty, E}\|\mathbf{q}\|_{2, E}\|\mathbf{v}\|_{1, E} .
$$

Furthermore, on simplicial elements, the above inequality also holds for all $\mathbf{q}, \mathbf{v} \in \mathbf{V}_{h}$.

Theorem 6.14. Assume that (5.18) holds, $K \in W^{1, \infty}, K^{-1} \in W_{\mathcal{T}_{h}}^{2, \infty}$, and the $H^{2}$ elliptic regularity condition (6.47) holds. Then, the pressure $p_{h}$ of the mortar MFMFE method (4.1)-(4.3) on simplicial elements, $h^{2}$-parallelograms, and regular $h^{2}$-parallelepipeds, satisfies

$$
\left\|Q_{h} p-p_{h}\right\| \lesssim \sum_{i=1}^{n}\left(H^{s+1 / 2}\|p\|_{s+1 / 2, \Omega_{i}}+h H\|\mathbf{u}\|_{1, \Omega_{i}}+h^{3 / 2} H^{1 / 2}\|\mathbf{u}\|_{2, \Omega_{i}}\right)+h^{r} H^{3 / 2}\|\mathbf{u}\|_{r+1 / 2},
$$

where $0<s \leq m+1$ and $0<r \leq 1$.

Proof. Consider an auxiliary problem

$$
\begin{aligned}
-\nabla \cdot(K \nabla \phi) & =p_{h}-Q_{h} p, & & \text { in } \Omega, \\
\phi & =0, & & \text { on } \partial \Omega .
\end{aligned}
$$

We assume that the problem is $H^{2}$-elliptic regular:

$$
\|\phi\|_{2} \lesssim\left\|Q_{h} p-p_{h}\right\| .
$$

Sufficient conditions for $(6.47)$ can be found in $[35,43]$. For example, it holds if the components of $K \in C^{0,1}(\bar{\Omega})$ and $\partial \Omega$ is smooth enough. By (5.7), (3.22), and (5.34),

$\sum_{i=1}^{n}\left(p-p_{h}, \nabla \cdot \Pi^{R T} \Pi_{0} K \nabla \phi\right)_{\Omega_{i}}=\sum_{i=1}^{n}\left(Q_{h} p-p_{h}, \nabla \cdot \Pi^{R T} \Pi_{0} K \nabla \phi\right)_{\Omega_{i}}=\sum_{i=1}^{n}\left(Q_{h} p-p_{h}, \nabla \cdot K \nabla \phi\right)_{\Omega_{i}}=\left\|Q_{h} p-p_{h}\right\|^{2}$.

Taking $\mathbf{v}=\Pi^{R T} \Pi_{0} K \nabla \phi \in \mathbf{V}_{h, 0}$ in (6.2),

$$
\left\|Q_{h} p-p_{h}\right\|^{2}=\left(K^{-1} \mathbf{u}, \mathbf{v}\right)-\left(K^{-1} \mathbf{u}_{h}, \mathbf{v}\right)_{Q}+\sum_{i=1}^{n}\left\langle p, \mathbf{v} \cdot \mathbf{n}_{i}\right\rangle_{\Gamma_{i}}
$$

We rewrite (6.48) as

$$
\left\|Q_{h} p-p_{h}\right\|^{2}=\left(K^{-1}(\mathbf{u}-\Pi \mathbf{u}), \mathbf{v}\right)-\left(K^{-1}\left(\mathbf{u}_{h}-\Pi \mathbf{u}\right), \mathbf{v}\right)_{Q}+\sigma\left(K^{-1} \Pi \mathbf{u}, \mathbf{v}\right)+\sum_{i=1}^{n}\left\langle p-\mathcal{P}_{H} p, \mathbf{v} \cdot \mathbf{n}_{i}\right\rangle_{\Gamma_{i}}
$$


where we have also used the weak continuity of $\mathbf{v}$, see (5.14). Using (3.24) and (5.37), we bound the first term on the right in (6.49) as

$$
\left(K^{-1}(\mathbf{u}-\Pi \mathbf{u}), \Pi^{R T} \Pi_{0} K \nabla \phi\right) \lesssim\left\|K^{-1}\right\|_{0, \infty}\|\mathbf{u}-\Pi \mathbf{u}\|\|K\|_{1, \infty}\|\phi\|_{2} .
$$

For the third term on the right in $(6.49),(6.44)$ implies that

$$
\begin{aligned}
\sigma\left(K^{-1} \Pi \mathbf{u}, \mathbf{v}_{h}\right) & \lesssim \sum_{E \in \mathcal{T}_{h}} h^{2}\left\|K^{-1}\right\|_{2, \infty, E}\|\Pi \mathbf{u}\|_{2, E}\left\|\Pi^{R T} \Pi_{0} K \nabla \phi\right\|_{1, E} \\
& \lesssim \sum_{i=1}^{n} h^{3 / 2} H^{1 / 2}\left\|K^{-1}\right\|_{2, \infty}\|K\|_{1, \infty}\|\mathbf{u}\|_{2, \Omega_{i}}\|\phi\|_{2},
\end{aligned}
$$

where we have used (5.5), (5.6), and (5.38). The fourth term on the right in (6.49) represents the mortar interface error, which can be written as

$$
\begin{aligned}
\sum_{i=1}^{n}\left\langle p-\mathcal{P}_{H} p, \Pi^{R T} \Pi_{0} K \nabla \phi \cdot \mathbf{n}_{i}\right\rangle_{\Gamma_{i}}= & \sum_{i=1}^{n}\left\langle p-\mathcal{P}_{H} p,\left(\Pi^{R T} \Pi_{0} K \nabla \phi-\Pi^{R T} K \nabla \phi\right) \cdot \mathbf{n}_{i}\right\rangle_{\Gamma_{i}} \\
& +\sum_{i=1}^{n}\left\langle p-\mathcal{P}_{H} p,\left(\Pi^{R T} K \nabla \phi-K \nabla \phi\right) \cdot \mathbf{n}_{i}\right\rangle_{\Gamma_{i}}+\sum_{i=1}^{n}\left\langle p-\mathcal{P}_{H} p, K \nabla \phi \cdot \mathbf{n}_{i}\right\rangle_{\Gamma_{i}} .
\end{aligned}
$$

Using (5.12), (5.41), (3.24), (5.35), and (5.33), we bound the first term on the right in (6.52) as

$$
\begin{aligned}
\sum_{i=1}^{n}\left(\left\langle p-\mathcal{P}_{H} p,\left(\Pi^{R T} \Pi_{0} K \nabla \phi-\Pi^{R T} K \nabla \phi\right) \cdot \mathbf{n}_{i}\right\rangle_{\Gamma_{i}}\right. & \lesssim \sum_{i=1}^{n}\left\|p-\mathcal{P}_{H} p\right\|_{\Gamma_{i}}\left\|\left(\Pi^{R T} \Pi_{0} K \nabla \phi-\Pi^{R T} \Pi K \nabla \phi\right) \cdot \mathbf{n}_{i}\right\|_{\Gamma_{i}} \\
& \lesssim \sum_{i=1}^{n} H^{s}\|p\|_{s, \Gamma_{i}} h^{-1 / 2}\left\|\Pi^{R T} \Pi_{0} K \nabla \phi-\Pi^{R T} \Pi K \nabla \phi\right\|_{\Omega_{i}} \\
& \lesssim \sum_{i=1}^{n} H^{s}\|p\|_{s, \Gamma_{i}} h^{-1 / 2}\left\|\Pi_{0} K \nabla \phi-\Pi K \nabla \phi\right\|_{\Omega_{i}} \\
& \lesssim \sum_{i=1}^{n} H^{s+1 / 2}\|K\|_{1, \infty}\|p\|_{s+1 / 2, \Omega_{i}}\|\phi\|_{2}, \quad 0<s \leq m+1 .
\end{aligned}
$$

Using (5.12), (5.13), and (5.33), we bound the second and third terms on the right in (6.52) as

$$
\begin{aligned}
\sum_{i=1}^{n}(\langle p & \left.\left.-\mathcal{P}_{H} p,\left(\Pi^{R T} K \nabla \phi-K \nabla \phi\right) \cdot \mathbf{n}_{i}\right\rangle_{\Gamma_{i}}+\left\langle p-\mathcal{P}_{H} p, K \nabla \phi \cdot \mathbf{n}_{i}\right\rangle_{\Gamma_{i}}\right) \\
& \lesssim \sum_{i=1}^{n}\left\|p-\mathcal{P}_{H} p\right\|_{\Gamma_{i}}\left\|\left(\Pi^{R T} K \nabla \phi-K \nabla \phi\right) \cdot \mathbf{n}_{i}\right\|_{\Gamma_{i}}+\sum_{i=1}^{n}\left\|p-\mathcal{P}_{H} p\right\|_{-1 / 2, \Gamma_{i}}\left\|K \nabla \phi \cdot \mathbf{n}_{i}\right\|_{1 / 2, \Gamma_{i}} \\
& \lesssim \sum_{i=1}^{n} H^{s+1 / 2}\|p\|_{s+1 / 2, \Omega_{i}}\|K\|_{1, \infty, \Omega_{i}}\|\phi\|_{2}, \quad 0<s \leq m+1
\end{aligned}
$$

It remains to estimate the second term in (6.49), which can be manipulated as

$$
\left(K^{-1}\left(\Pi \mathbf{u}-\mathbf{u}_{h}\right), \Pi^{R T} \Pi_{0} K \nabla \phi\right)_{Q}=\left(K^{-1}\left(\Pi \mathbf{u}-\mathbf{u}_{h}\right), \Pi^{R T}\left(\Pi_{0}-\Pi\right) K \nabla \phi\right)_{Q}+\left(K^{-1}\left(\Pi \mathbf{u}-\mathbf{u}_{h}\right), \Pi^{R T} K \nabla \phi\right)_{Q}
$$


The first term on the right above can be bounded using (3.24) and (5.35):

$$
\left(K^{-1}\left(\Pi \mathbf{u}-\mathbf{u}_{h}\right), \Pi^{R T}\left(\Pi_{0}-\Pi\right) K \nabla \phi\right)_{Q} \lesssim\left\|\Pi \mathbf{u}-\mathbf{u}_{h}\right\| h^{1 / 2} H^{1 / 2}\|K\|_{1, \infty}\|\phi\|_{2} .
$$

For the second term on the right in (6.55) we write

$$
\begin{aligned}
\left(K^{-1}\left(\Pi \mathbf{u}-\mathbf{u}_{h}\right), \Pi^{R T} K \nabla \phi\right)_{Q}= & \left(\left(K^{-1}-\bar{K}^{-1}\right)\left(\Pi \mathbf{u}-\mathbf{u}_{h}\right), \Pi^{R T} K \nabla \phi\right)_{Q}+\left(\bar{K}^{-1}\left(\Pi \mathbf{u}-\mathbf{u}_{h}\right), \Pi^{R T}(K-\bar{K}) \nabla \phi\right)_{Q} \\
& +\left(\bar{K}^{-1}\left(\Pi \mathbf{u}-\mathbf{u}_{h}\right), \Pi^{R T} \bar{K}\left(\nabla \phi-\nabla \phi_{1}\right)\right)_{Q}+\left(\bar{K}^{-1}\left(\Pi \mathbf{u}-\mathbf{u}_{h}\right), \Pi^{R T} \bar{K} \nabla \phi_{1}\right)_{Q}
\end{aligned}
$$

where $\bar{K}$ denotes the $L^{2}$-projection of $K$ onto the space of constant tensors and $\phi_{1}$ is a linear approximation to $\phi$ such that [18]

$$
\left\|\phi-\phi_{1}\right\|_{E} \lesssim h^{2}\|\phi\|_{2, E}, \quad\left\|\phi-\phi_{1}\right\|_{1, E} \lesssim h\|\phi\|_{2, E} .
$$

Using (5.6) and (1.4), the first term on the right in (6.57) can be bounded as

$$
\left(\left(K^{-1}-\bar{K}^{-1}\right)\left(\Pi \mathbf{u}-\mathbf{u}_{h}\right), \Pi^{R T} K \nabla \phi\right)_{Q, E} \lesssim h \frac{\|K\|_{1, \infty, E}^{2}}{k_{0}^{2}}\left\|\Pi \mathbf{u}-\mathbf{u}_{h}\right\|_{E}\|\phi\|_{2, E} .
$$

For the second and third terms on the right in (6.57), we use the inequality, for any $\mathbf{q} \in H^{1}(E)^{d}$,

$$
\left\|\Pi^{R T} \mathbf{q}\right\|_{E} \leq\left\|\Pi^{R T} \mathbf{q}-\mathbf{q}\right\|_{E}+\|\mathbf{q}\|_{E} \lesssim\left(h\|\mathbf{q}\|_{1, E}+\|\mathbf{q}\|_{E}\right),
$$

combined with (5.6) to obtain

$$
\left(\bar{K}^{-1}\left(\Pi \mathbf{u}-\mathbf{u}_{h}\right), \Pi^{R T}(K-\bar{K}) \nabla \phi\right)_{Q, E} \lesssim h \frac{\|K\|_{1, \infty, E}}{k_{0}}\left\|\Pi \mathbf{u}-\mathbf{u}_{h}\right\|_{E}\|\phi\|_{2, E},
$$

and

$$
\left(\bar{K}^{-1}\left(\Pi \mathbf{u}-\mathbf{u}_{h}\right), \Pi^{R T} \bar{K}\left(\nabla \phi-\nabla \phi_{1}\right)\right)_{Q} \lesssim h \frac{\|K\|_{0, \infty, E}}{k_{0}}\left\|\Pi \mathbf{u}-\mathbf{u}_{h}\right\|_{E}\|\phi\|_{2, E},
$$

where we have also used (6.58) in (6.61). Finally, for the last term in (6.57) we write as

$$
\left(\bar{K}^{-1}\left(\Pi \mathbf{u}-\mathbf{u}_{h}\right), \Pi^{R T} \bar{K} \nabla \phi_{1}\right)_{Q}=\left(\bar{K}^{-1}\left(\Pi \mathbf{u}-\mathbf{u}_{h}\right), \Pi^{R T} \bar{K} \nabla \phi_{1}-\bar{K} \nabla \phi_{1}\right)_{Q}+\left(\Pi \mathbf{u}-\mathbf{u}_{h}, \nabla \phi_{1}\right)_{Q} .
$$

The first term on the right in (6.62) can be bounded as

$$
\begin{aligned}
\left(\bar{K}^{-1}\left(\Pi \mathbf{u}-\mathbf{u}_{h}\right), \Pi^{R T} \bar{K} \nabla \phi_{1}-\bar{K} \nabla \phi_{1}\right)_{Q} & \lesssim \sum_{E \mathcal{T}_{h}} \frac{h}{k_{0}}\left\|\Pi \mathbf{u}-\mathbf{u}_{h}\right\|_{E}\left\|\bar{K} \nabla \phi_{1}\right\|_{E} \\
& \lesssim \sum_{E \mathcal{T}_{h}} h \frac{k_{1}}{k_{0}}\left\|\Pi \mathbf{u}-\mathbf{u}_{h}\right\|_{E}\left(\left\|\nabla \phi-\nabla \phi_{1}\right\|_{E}+\|\nabla \phi\|_{E}\right) \\
& \lesssim \sum_{E \mathcal{T}_{h}} h \frac{k_{1}}{k_{0}}\left\|\Pi \mathbf{u}-\mathbf{u}_{h}\right\|_{E}\|\phi\|_{2, E},
\end{aligned}
$$

where we have used (3.32), (5.2), and (6.58). By mapping to the reference element, the second term on the right in $(6.62)$ has been shown in $[39,56]$ that

$$
\left(\Pi \mathbf{u}-\mathbf{u}_{h}, \nabla \phi_{1}\right)_{Q}=R+\sum_{E \in \mathcal{T}_{h}}\left(\Pi^{R T}\left(\Pi \mathbf{u}-\mathbf{u}_{h}\right), \nabla \phi_{1}\right)_{E},
$$


where

$$
|R| \lesssim \sum_{E \in \mathcal{T}_{h}} h\left\|\Pi \mathbf{u}-\mathbf{u}_{h}\right\|_{E}\|\phi\|_{2, E}
$$

For the second term on the right in (6.64), using that $\phi$ and $\mathbf{u} \cdot \mathbf{n}$ are well defined on the element faces, as well as $(3.23),(6.8),(6.9)$, and (5.40), we have

$$
\begin{aligned}
\sum_{E \in \mathcal{T}_{h}}\left(\Pi^{R T}\left(\Pi \mathbf{u}-\mathbf{u}_{h}\right), \nabla \phi_{1}\right)_{E} & =\sum_{E \in \mathcal{T}_{h}}\left(\Pi^{R T}\left(\Pi \mathbf{u}-\mathbf{u}_{h}\right), \nabla\left(\phi_{1}-\phi\right)\right)_{E}+\sum_{i=1}^{n}\left\langle\Pi^{R T}\left(\Pi \mathbf{u}-\mathbf{u}_{h}\right) \cdot \mathbf{n}_{i}, \phi-\mathcal{I}_{H}^{c} \phi\right\rangle_{\partial \Omega_{i}} \\
& \lesssim \sum_{E \in \mathcal{T}_{h}} h\left\|\Pi \mathbf{u}-\mathbf{u}_{h}\right\|_{E}\|\phi\|_{2, E}+\sum_{i=1}^{n}\left\|\Pi^{R T}\left(\Pi \mathbf{u}-\mathbf{u}_{h}\right)\right\|_{H\left(\operatorname{div}, \Omega_{i}\right)}\left\|\phi-\mathcal{I}_{H}^{c} \phi\right\|_{1 / 2, \partial \Omega_{i}} \\
& \lesssim \sum_{E \in \mathcal{T}_{h}} h\left\|\Pi \mathbf{u}-\mathbf{u}_{h}\right\|_{E}\|\phi\|_{2, E}+\sum_{i=1}^{n}\left\|\Pi \mathbf{u}-\mathbf{u}_{h}\right\|_{\Omega_{i}} H\|\phi\|_{2, \Omega_{i}}
\end{aligned}
$$

where we have also used the approximation bounds (6.58) and (5.11). The proof is completed by a combination of (6.49)-(6.55), (6.56)-(6.66), (6.47), (5.2), (5.4), and (6.7).

\section{A domain DeCOMposition Formulation}

\subsection{Reduction to a mortar interface problem}

Following $[11,12,34]$, we reduce the global multiscale system (4.1)-(4.3) to a coarse scale interface problem for the mortar pressure. The resulting interface problem is symmetric and positive definite and can be solved using a preconditioned conjugate gradient $(\mathrm{CG})$ method.

Define a bilinear form $d_{H}: L^{2}(\Gamma) \times L^{2}(\Gamma) \rightarrow \mathbb{R}$ for $\lambda, \mu \in L^{2}(\Gamma)$ by

$$
d_{H}(\lambda, \mu)=\sum_{i=1}^{n} d_{H, i}(\lambda, \mu)=-\sum_{i=1}^{n}\left\langle\Pi^{R T} \mathbf{u}_{h}^{*}(\lambda) \cdot \mathbf{n}_{i}, \mu\right\rangle_{\Gamma_{i}},
$$

where $\left(\mathbf{u}_{h}^{*}(\lambda), p_{h}^{*}(\lambda)\right) \in \mathbf{V}_{h} \times W_{h}$ solve, for $1 \leq i \leq n$,

$$
\begin{aligned}
\left(K^{-1} \mathbf{u}_{h}^{*}(\lambda), \mathbf{v}\right)_{Q, \Omega_{i}}-\left(p_{h}^{*}(\lambda), \nabla \cdot \mathbf{v}\right)_{\Omega_{i}} & =-\left\langle\lambda, \Pi^{R T} \mathbf{v} \cdot \mathbf{n}_{i}\right\rangle_{\Gamma_{i}}, & & \mathbf{v} \in \mathbf{V}_{h, i}, \\
\left(\nabla \cdot \mathbf{u}_{h}^{*}(\lambda), w\right)_{\Omega_{i}} & =0, & w & \in W_{h, i} .
\end{aligned}
$$

The above subdomain problems represent the elimination of the interior degrees of freedom in forming the Schur complement for the mortar Lagrange multiplier.

Define a linear functional $g_{H}: L^{2}(\Gamma) \rightarrow \mathbb{R}$ by

$$
g_{H}(\mu)=\sum_{i=1}^{n} g_{H, i}(\mu)=\sum_{i=1}^{n}\left\langle\Pi^{R T} \overline{\mathbf{u}}_{h} \cdot \mathbf{n}_{i}, \mu\right\rangle_{\Gamma_{i}},
$$

where $\left(\overline{\mathbf{u}}_{h}, \bar{p}_{h}\right) \in \mathbf{V}_{h} \times W_{h}$ solve, for $1 \leq i \leq n$,

$$
\begin{aligned}
\left(K^{-1} \overline{\mathbf{u}}_{h}(\lambda), \mathbf{v}\right)_{Q, \Omega_{i}}- & \left(\bar{p}_{h}(\lambda), \nabla \cdot \mathbf{v}\right)_{\Omega_{i}}=-\left\langle g, \Pi^{R T} \mathbf{v} \cdot \mathbf{n}_{i}\right\rangle_{\partial \Omega_{i} / \Gamma_{i}}, & \mathbf{v} & \in \mathbf{V}_{h, i}, \\
\left(\nabla \cdot \overline{\mathbf{u}}_{h}(\lambda), w\right)_{\Omega_{i}} & =f, & w & \in W_{h, i} .
\end{aligned}
$$

It is easy to show that solving (4.1)-(4.3) is equivalent to solving the interface problem for $\lambda_{H} \in \Lambda_{H}$,

$$
d_{H}\left(\lambda_{H}, \mu\right)=g_{H}(\mu), \quad \mu \in \Lambda_{H},
$$

and calculating

$$
\mathbf{u}_{h}=\mathbf{u}_{h}^{*}\left(\lambda_{H}\right)+\overline{\mathbf{u}}_{h}, \quad p_{h}=p_{h}^{*}\left(\lambda_{H}\right)+\bar{p}_{h}
$$




\subsection{Mortar pressure error estimate}

Lemma 7.1. The interface bilinear form $d_{H}(\cdot, \cdot)$ is symmetric and positive semi-definite on $L^{2}(\Gamma)$. If $(5.23)$ holds, then $d_{H}(\cdot, \cdot)$ is positive definite on $\Lambda_{H}$.

Proof. Let $\mathbf{v}=\mathbf{u}_{h}^{*}(\mu)$ in (7.1) for some $\mu \in L^{2}(\Gamma)$ to obtain

$$
d_{H, i}(\mu, \lambda)=-\left\langle\Pi^{R T} \mathbf{u}_{h}^{*}(\mu) \cdot \mathbf{n}_{i}, \lambda\right\rangle_{\Gamma_{i}}=\left(K^{-1} \mathbf{u}_{h}^{*}(\lambda), \mathbf{u}_{h}^{*}(\mu)\right)_{Q, \Omega_{i}},
$$

which shows that $d_{H}(\cdot, \cdot)$ is symmetric and

$$
d_{H, i}(\mu, \mu)=\left(K^{-1} \mathbf{u}_{h}^{*}(\mu), \mathbf{u}_{h}^{*}(\mu)\right)_{Q, \Omega_{i}} \geq 0 .
$$

For $\mu \in \Lambda_{H}$, if (5.23) holds, the argument from Lemma 6.1 shows that $d_{H}(\mu, \mu)=0$ implies $\mu=0$.

Let $\|\cdot\|_{d_{H}}$ be the seminorm induced by $d_{H}(\cdot, \cdot)$ on $L^{2}(\Gamma)$ :

$$
\|\mu\|_{d_{H}}=d_{H}(\mu, \mu)^{1 / 2}, \quad \mu \in L^{2}(\Gamma) .
$$

The proof of the next result is similar to the proof of Theorem 4.4 in [12].

Theorem 7.2. For the mortar pressure $\Lambda_{H}$ of the mortar MFMFE method (4.1)-(4.3) on simplicial grids, $h^{2}$-parallelograms, and $h^{2}$-parallelepipeds, if (5.18) holds, then

$$
\left\|p-\lambda_{H}\right\|_{d_{H}} \lesssim\left\|\mathbf{u}-\mathbf{u}_{h}\right\|+\sum_{i=1}^{n} h\|\mathbf{u}\|_{1, \Omega_{i}} .
$$

Proof. Let, for $\mu \in L^{2}(\Gamma)$,

$$
\mathbf{u}_{h}(\mu)=\mathbf{u}_{h}^{*}(\mu)+\overline{\mathbf{u}}_{h}, \quad p_{h}(\mu)=p_{h}^{*}(\mu)+\bar{p}_{h},
$$

and note that $\left(\mathbf{u}_{h}(\mu), p_{h}(\mu)\right) \in \mathbf{V}_{h} \times W_{h}$ satisfies

$$
\begin{array}{rlrl}
\left(K^{-1} \mathbf{u}_{h}(\mu), \mathbf{v}\right)_{Q, \Omega_{i}}- & \left(p_{h}(\mu), \nabla \cdot \mathbf{v}\right)_{\Omega_{i}}=-\left\langle\mu, \Pi^{R T} \mathbf{v} \cdot \mathbf{n}\right\rangle_{\Gamma_{i}}-\left\langle g, \Pi^{R T} \mathbf{v} \cdot \mathbf{n}\right\rangle_{\partial \Omega_{i} / \Gamma_{i}}, & \mathbf{v} \in \mathbf{V}_{h, i} \\
\left(\nabla \cdot \mathbf{u}_{h}(\mu), w\right)_{\Omega_{i}} & =(f, w)_{\Omega_{i}}, & w & \in W_{h, i} .
\end{array}
$$

In particular $\mathbf{u}_{h}\left(\lambda_{H}\right)=\mathbf{u}_{h}$ and $p_{h}\left(\lambda_{H}\right)=p_{h}$. From (7.7), the linearity of $\mathbf{u}_{h}^{*}(\cdot)$ implies that

$$
\begin{aligned}
\left\|p-\lambda_{H}\right\|_{d_{H}} & \lesssim\left\|\mathbf{u}_{h}^{*}(p)-\mathbf{u}_{h}^{*}\left(\lambda_{H}\right)\right\|=\left\|\mathbf{u}_{h}(p)-\mathbf{u}_{h}\left(\lambda_{H}\right)\right\| \\
& =\left\|\mathbf{u}_{h}(p)-\mathbf{u}_{h}\right\| \leq\left\|\mathbf{u}_{h}(p)-\mathbf{u}\right\|+\left\|\mathbf{u}-\mathbf{u}_{h}\right\|,
\end{aligned}
$$

where we have also used norm equivalence (3.31) and (7.9). The estimate (7.8) follows from first order convergence of the MFMFE method on a single block [39, 56]:

$$
\left\|\mathbf{u}_{h}(p)-\mathbf{u}\right\|_{\Omega_{i}} \lesssim h\|\mathbf{u}\|_{1, \Omega_{i}}
$$

\subsection{Multiscale flux basis implementation}

In the original implementation of the mortar mixed finite element method $[11,12]$, the action of the interface operator (7.5) in each conjugate gradient iteration is computed by solving subdomain problems. Alternatively, a multiscale flux basis with respect to the mortar variables can be computed before the start of the interface iteration [32]. The computation of these basis functions requires solving a fixed number (equal to the number of mortar degrees of freedom per subdomain) of Dirichlet subdomain problems. Then, an inexpensive linear combination of the multiscale flux basis functions replaces subdomain solves during the interface iteration. 
Following [32], let $\left\{\phi_{H, i}^{(k)}\right\}_{k=1}^{N_{H, i}}$ denote the basis function of mortar space $\Lambda_{H, i}$, where $N_{H, i}$ is the number of mortar degrees of freedom on subdomain $\Omega_{i}$. Then for $\lambda_{H, i} \in \Lambda_{H, i}$, we have

$$
\lambda_{H, i}=\sum_{k=1}^{N_{H, i}} \lambda_{H, i}^{(k)} \phi_{H, i}^{(k)} .
$$

The computation of multiscale flux basis function $\psi_{H, i}^{(k)}=D_{H, i} \phi_{H, i}^{(k)}$ with respect to the mortar basis functions $\phi_{H, i}^{(k)}$ is as follows.

For $k=1,2, \ldots, N_{H, i}$,

(1) project $\phi_{H, i}^{(k)}$ on the subdomain boundary:

$$
\mathcal{Q}_{h, i}^{R T} \phi_{H, i}^{(k)}=\gamma_{i}^{(k)}
$$

(2) solve the subdomain problem:

$$
\begin{aligned}
\left(K^{-1} \mathbf{u}_{h}^{*}\left(\gamma_{i}^{(k)}\right), \mathbf{v}\right)_{Q, \Omega_{i}}-\left(p_{h}^{*}\left(\gamma_{i}^{(k)}\right), \nabla \cdot \mathbf{v}\right)_{\Omega_{i}}=-\left\langle\gamma_{i}^{(k)}, \Pi^{R T} \mathbf{v} \cdot \mathbf{n}_{i}\right\rangle_{\Gamma_{i}}, & \mathbf{v} \in \mathbf{V}_{h, i} \\
\left(\nabla \cdot \mathbf{u}_{h}^{*}\left(\gamma_{i}^{(k)}\right), w\right)_{\Omega_{i}}=0, & w \in W_{h, i}
\end{aligned}
$$

(3) project the boundary flux onto the mortar space:

$$
\psi_{H, i}^{(k)}=-\left(\mathcal{Q}_{h, i}^{R T}\right)^{T} \Pi^{R T} \mathbf{u}_{h}^{*}\left(\gamma_{i}^{(k)}\right) \cdot \mathbf{n}_{i} .
$$

Once the multiscale flux basis functions are constructed, the action of interface operator $D_{H, i}$, defined by

$$
\left\langle D_{H, i} \lambda_{H, i}, \mu\right\rangle_{\Gamma_{i}}=d_{H, i}(\lambda, \mu)=-\left\langle\Pi^{R T} \mathbf{u}_{h}^{*}(\lambda) \cdot \mathbf{n}_{i}, \mu\right\rangle_{\Gamma_{i}}, \quad \forall \mu \in \Lambda_{H, i},
$$

simply involves a linear combination of the multiscale basis functions:

$$
D_{H, i} \lambda_{H, i}=D_{H, i}\left(\sum_{k=1}^{N_{H, i}} \lambda_{H, i}^{(k)} \phi_{H, i}^{(k)}\right)=\sum_{k=1}^{N_{H, i}} \lambda_{H, i}^{(k)} D_{H, i} \phi_{H, i}^{(k)}=\sum_{k=1}^{N_{H, i}} \lambda_{H, i}^{(k)} \psi_{H, i}^{(k)} .
$$

For the computational efficiency of multiscale mortar flux basis approach, we refer to [32].

\section{NUMERICAL EXPERIMENTS}

In this section, we confirm our theoretical results by presenting several numerical examples on rectangular, $h^{2}$-parallelogram, cuboid, and regular $h^{2}$-parallelepiped grids. The first two examples are for two dimensional problems. The computational domain of the first example is the unit square. In the second example, we use a global smooth mapping to generate irregular domain from the unit square. The third example is on the unit cube. The fourth example tests $h^{2}$-parallelepiped grids on irregular domain obtained via a mapping of a unit cube. We also apply the method to solve a problem with a highly heterogeneous permeability, and compare the fine scale and multiscale solutions.

In the convergence tests, the domain is divided into four subdomains for the 2D examples and eight subdomains for the $3 \mathrm{D}$ examples with interfaces along the $x=0.5$ and $y=0.5$ (and $z=0.5$ for 3D examples) lines (planes). For the boundary conditions, we choose Dirichlet on $x=0$ and $x=1$ and Neumann on the rest of the boundary. We consider both matching and non-matching girds.

We employ the conjugate gradient method to solve the interface problem (7.5) arising from the domain decomposition algorithm in Section 7. The stopping criteria for the conjugate gradient iteration is the relative 
TABLE 1. Theoretical convergence rates for linear and quadratic mortars.

\begin{tabular}{c|ccccc}
\hline$m$ & $\mathrm{H}$ & $\left\|p-p_{h}\right\|$ & $\left\|\mathbf{u}-\mathbf{u}_{h}\right\|$ & $\left\|p-p_{h}\right\|$ & $\left\|\mathbf{u}-\Pi^{R T} \mathbf{u}_{h}\right\|$ \\
\hline 1 & $2 h$ & $h$ & $h$ & $h^{2}$ & $h^{1.5}$ \\
2 & $h^{1 / 2}$ & $h$ & $h$ & $h^{1.5}$ & $h^{1.25}$ \\
\hline
\end{tabular}

residual error to be smaller than $10^{-6}$. In each conjugate iteration, we perform a linear combination of the multiscale flux basis functions to compute the action of the operator $D_{H, i}$, as described in Section 7.3. In the numerical examples, we report the numerical error between the computed solution and exact solution, as well as the number of conjugate gradient iterations.

The rectangular and cuboid meshes on each level are generated by uniform refinements of each subdomain grids and the mortar grid. For the coarsest matching grid, the mesh on each subdomain is $2 \times 2$ in $2 \mathrm{D}$ and $2 \times 2 \times 2$ in $3 \mathrm{D}$. For the coarsest nonmatching grid in $2 \mathrm{D}$, we use $2 \times 2$ or $3 \times 3$ alternated in a checkerboard fashion. We test both linear mortars $(m=1)$ and quadratic mortars $(m=2)$. The mortar spaces can be continuous or discontinuous. The coarsest mortar grids on all interfaces have one element, so $H=1 / 2$. For the linear mortars, we refine by half both subdomain and mortar meshes, which gives $H=2 h$ on each level. For the quadratic mortars, we refine the subdomain meshes by four and mortar meshes by half, which gives $H=h^{1 / 2}$. The choice is motivated by balancing the fine scale and coarse scale error terms in the theoretical convergence results, see also Table 1.

The $h^{2}$-parallelogram and $h^{2}$-parallelepiped meshes on each level are obtained by a global mapping of the rectangular and cuboid meshes generated by the above procedure.

The convergence rates are reported for each level of grid refinement. Table 1 shows convergence rates predicted by the theory for linear and quadratic mortars. Note that by appropriately choosing the coarse scale size and polynomial degree, the method exhibits fine scale convergence. Higher order mortars allow for coarser mortar grids.

The errors $\left\|p-p_{h}\right\|$ and $\left\|\mathbf{u}-\mathbf{u}_{h}\right\|$ are computed by the element-by-element trapezoidal rule. The pressure error $\left\|p-p_{h}\right\|$ is in the discrete $L^{2}$-norm computed by the midpoint quadrature rule: $\left\|p-p_{h}\right\|^{2} \equiv \sum_{E \in \mathcal{T}_{h}}|E|(p-$ $\left.p_{h}\right)^{2}\left(m_{e}\right)$, where $m_{e}$ is the center of mass of element $E$. Since $\left\|p-Q_{h} p\right\| \lesssim h^{2}$, we have $\left\|p-p_{h}\right\| \leq\left\|Q_{h} p-p_{h}\right\|+$ $\left\|p-Q_{h} p\right\|=\left\|Q_{h} p-p_{h}\right\|+O\left(h^{2}\right)$. This gives the superconvergence result for $\left\|p-p_{h}\right\|$ as well. The velocity error $\left\|\mathbf{u}-\Pi^{R T} \mathbf{u}_{h}\right\|$ is defined in (6.32).

\subsection{Example 1: rectangular mesh}

In the first example, we take the domain to be the unit square and solve the problem on rectangular grids with given analytic solution

$$
p(x, y)=x^{3} y^{4}+x^{2}+\sin (x y) \cos (y)
$$

and a full permeability tensor

$$
K=\left(\begin{array}{cc}
(x+1)^{2}+y^{2} & \sin (x y) \\
\sin (x y) & (x+1)^{2}
\end{array}\right) .
$$

We present four cases with either linear or quadratic mortars on matching or nonmatching grids. Convergence rates for various norms are given in Tables $2-5$. The observed convergence rates are at least as good as the theory predicts. In all four cases, we obtain first order convergence for both the pressure error $\left\|p-p_{h}\right\|$ and the velocity error $\left\|\mathbf{u}-\mathbf{u}_{h}\right\|$. The discrete pressure error $\left\|p-p_{h}\right\|$ is superconvergent of order $\mathcal{O}\left(h^{2}\right)$ for all four cases, even though Theorem 6.14 predicts only $\mathcal{O}\left(h^{1.5}\right)$ for quadratic mortars. Theorem 6.9 predicts that the discrete velocity error $\left\|\mathbf{u}-\Pi^{R T} \mathbf{u}_{h}\right\|$ is superconvergent of order $\mathcal{O}\left(h^{1.25}\right)$ for quadratic mortars and $\mathcal{O}\left(h^{1.5}\right)$ for linear mortars. We observe convergence of order $\mathcal{O}\left(h^{1.5}\right)$ or higher in all four cases.

In terms of the computational cost, quadratic mortars are more efficient than linear mortars. Continuous linear mortars need $50 \mathrm{CG}$ iterations with $h=1 / 256$, see Table 2 , while continuous quadratic mortars require 
TABLE 2. Example 1: continuous linear mortars on matching grids.

\begin{tabular}{c|ccccccccc}
\hline $1 / h$ & $\left\|p-p_{h}\right\|$ & & $\left\|\mathbf{u}-\mathbf{u}_{h}\right\|$ & & $\left\|p-p_{h}\right\|$ & & $\left\|\mathbf{u}-\Pi^{R T} \mathbf{u}_{h}\right\|$ & & CGiter \\
\hline 4 & $2.53 \mathrm{E}-01$ & - & $1.06 \mathrm{E}+00$ & - & $5.39 \mathrm{E}-02$ & - & $1.27 \mathrm{E}-01$ & - & 8 \\
8 & $1.21 \mathrm{E}-01$ & 1.06 & $5.23 \mathrm{E}-01$ & 1.02 & $1.38 \mathrm{E}-02$ & 1.97 & $3.10 \mathrm{E}-02$ & 2.03 & 11 \\
16 & $5.96 \mathrm{E}-02$ & 1.02 & $2.57 \mathrm{E}-01$ & 1.03 & $3.46 \mathrm{E}-03$ & 2.00 & $7.66 \mathrm{E}-03$ & 2.02 & 14 \\
32 & $2.97 \mathrm{E}-02$ & 1.00 & $1.27 \mathrm{E}-01$ & 1.02 & $8.66 \mathrm{E}-04$ & 2.00 & $1.92 \mathrm{E}-03$ & 2.00 & 18 \\
64 & $1.48 \mathrm{E}-02$ & 1.00 & $6.34 \mathrm{E}-02$ & 1.00 & $2.16 \mathrm{E}-04$ & 2.00 & $4.80 \mathrm{E}-04$ & 2.00 & 26 \\
128 & $7.42 \mathrm{E}-03$ & 1.00 & $3.16 \mathrm{E}-02$ & 1.00 & $5.41 \mathrm{E}-05$ & 2.00 & $1.20 \mathrm{E}-04$ & 2.00 & 36 \\
256 & $3.71 \mathrm{E}-03$ & 1.00 & $1.58 \mathrm{E}-02$ & 1.00 & $1.36 \mathrm{E}-05$ & 1.99 & $3.67 \mathrm{E}-05$ & 1.71 & 50 \\
\hline
\end{tabular}

TABLE 3. Example 1: continuous quadratic mortars on matching grids.

\begin{tabular}{c|ccccccccc}
\hline $1 / h$ & $\left\|p-p_{h}\right\|$ & & $\left\|\mathbf{u}-\mathbf{u}_{h}\right\|$ & & $\left\|p-p_{h}\right\|$ & & $\left\|\mathbf{u}-\Pi^{R T} \mathbf{u}_{h}\right\|$ & & CGiter \\
\hline 4 & $2.53 \mathrm{E}-01$ & - & $1.06 \mathrm{E}+00$ & - & $5.39 \mathrm{E}-02$ & - & $1.27 \mathrm{E}-01$ & - & 8 \\
16 & $5.96 \mathrm{E}-02$ & 1.04 & $2.57 \mathrm{E}-01$ & 1.02 & $3.46 \mathrm{E}-03$ & 1.98 & $7.69 \mathrm{E}-03$ & 2.02 & 15 \\
64 & $1.48 \mathrm{E}-02$ & 1.00 & $6.34 \mathrm{E}-02$ & 1.01 & $2.16 \mathrm{E}-04$ & 2.00 & $5.71 \mathrm{E}-04$ & 1.88 & 22 \\
256 & $3.71 \mathrm{E}-03$ & 1.00 & $1.58 \mathrm{E}-02$ & 1.00 & $1.36 \mathrm{E}-05$ & 1.99 & $7.61 \mathrm{E}-05$ & 1.45 & 33 \\
\hline
\end{tabular}

TABLE 4. Example 1: discontinuous linear mortars on nonmatching grids.

\begin{tabular}{c|ccccccccc}
\hline $1 / h$ & $\left\|p-p_{h}\right\|$ & & $\left\|\mathbf{u}-\mathbf{u}_{h}\right\|$ & & $\left\|p-p_{h}\right\|$ & & $\left\|\mathbf{u}-\Pi^{R T} \mathbf{u}_{h}\right\|$ & & CGiter \\
\hline 4 & $1.97 \mathrm{E}-01$ & - & $7.59 \mathrm{E}-01$ & - & $3.59 \mathrm{E}-02$ & - & $1.61 \mathrm{E}-01$ & - & 8 \\
8 & $9.59 \mathrm{E}-02$ & 1.04 & $3.69 \mathrm{E}-01$ & 1.04 & $9.18 \mathrm{E}-03$ & 1.97 & $5.02 \mathrm{E}-02$ & 1.68 & 13 \\
16 & $4.76 \mathrm{E}-02$ & 1.01 & $1.81 \mathrm{E}-01$ & 1.03 & $2.31 \mathrm{E}-03$ & 1.99 & $1.54 \mathrm{E}-02$ & 1.70 & 19 \\
32 & $2.37 \mathrm{E}-02$ & 1.01 & $8.99 \mathrm{E}-02$ & 1.01 & $5.79 \mathrm{E}-04$ & 2.00 & $4.82 \mathrm{E}-03$ & 1.68 & 28 \\
64 & $1.19 \mathrm{E}-02$ & 0.99 & $4.48 \mathrm{E}-02$ & 1.00 & $1.45 \mathrm{E}-04$ & 2.00 & $1.56 \mathrm{E}-03$ & 1.63 & 41 \\
128 & $5.93 \mathrm{E}-03$ & 1.00 & $2.24 \mathrm{E}-02$ & 1.00 & $3.63 \mathrm{E}-05$ & 2.00 & $5.18 \mathrm{E}-04$ & 1.60 & 58 \\
256 & $2.97 \mathrm{E}-03$ & 1.00 & $1.12 \mathrm{E}-02$ & 1.00 & $9.10 \mathrm{E}-06$ & 2.00 & $1.77 \mathrm{E}-04$ & 1.55 & 85 \\
\hline
\end{tabular}

TABLE 5. Example 1: discontinuous quadratic mortars on nonmatching grids.

\begin{tabular}{c|ccccccccc}
\hline $1 / h$ & $\left\|p-p_{h}\right\|$ & & $\left\|\mathbf{u}-\mathbf{u}_{h}\right\|$ & & $\left\|p-p_{h}\right\|$ & & $\left\|\mathbf{u}-\Pi^{R T} \mathbf{u}_{h}\right\|$ & & CGiter \\
\hline 4 & $1.97 \mathrm{E}-01$ & - & $7.54 \mathrm{E}-01$ & - & $3.64 \mathrm{E}-02$ & - & $1.45 \mathrm{E}-01$ & - & 11 \\
16 & $4.76 \mathrm{E}-02$ & 1.02 & $1.81 \mathrm{E}-01$ & 1.03 & $2.32 \mathrm{E}-03$ & 1.99 & $1.14 \mathrm{E}-02$ & 1.83 & 18 \\
64 & $1.19 \mathrm{E}-02$ & 1.00 & $4.48 \mathrm{E}-02$ & 1.01 & $1.45 \mathrm{E}-04$ & 2.00 & $8.46 \mathrm{E}-04$ & 1.88 & 33 \\
256 & $2.97 \mathrm{E}-03$ & 1.00 & $1.12 \mathrm{E}-02$ & 1.00 & $9.12 \mathrm{E}-06$ & 2.00 & $7.75 \mathrm{E}-05$ & 1.72 & 58 \\
\hline
\end{tabular}

only 33 iterations on the same fine mesh level, as shown in Table 3. Similarly for the discontinuous case in Tables 4, 5, the CG iteration number with quadratic mortars is smaller than that with linear mortars. This can be explained by the much coarser mortar grids used with quadratic mortars. The computed velocity and pressure with discontinuous linear and quadratic mortars on the same nonmatching grids are given in Figure 3. The numerical errors are given in Figure 4 accordingly. The errors are comparable, although a somewhat smaller velocity error along the interfaces is observed for quadratic mortars. 

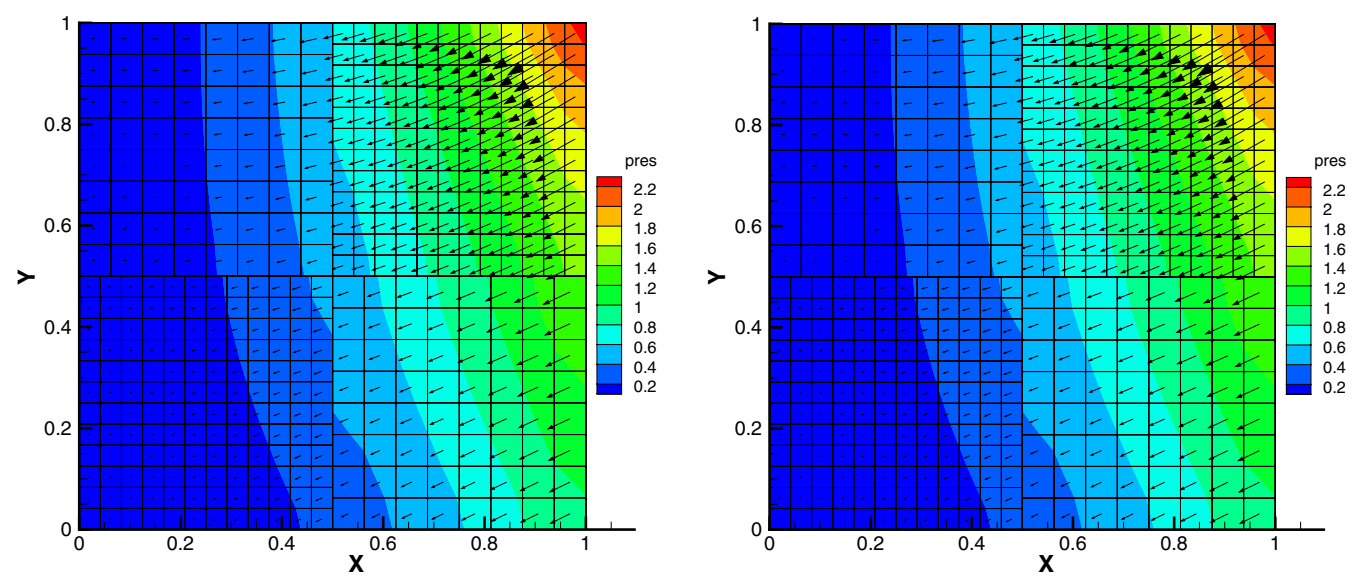

FigURE 3. Multiscale mortar MFMFE solution on nonmatching rectangular grids in Example 1: discontinuous linear (left) and discontinuous quadratic (right) mortars.
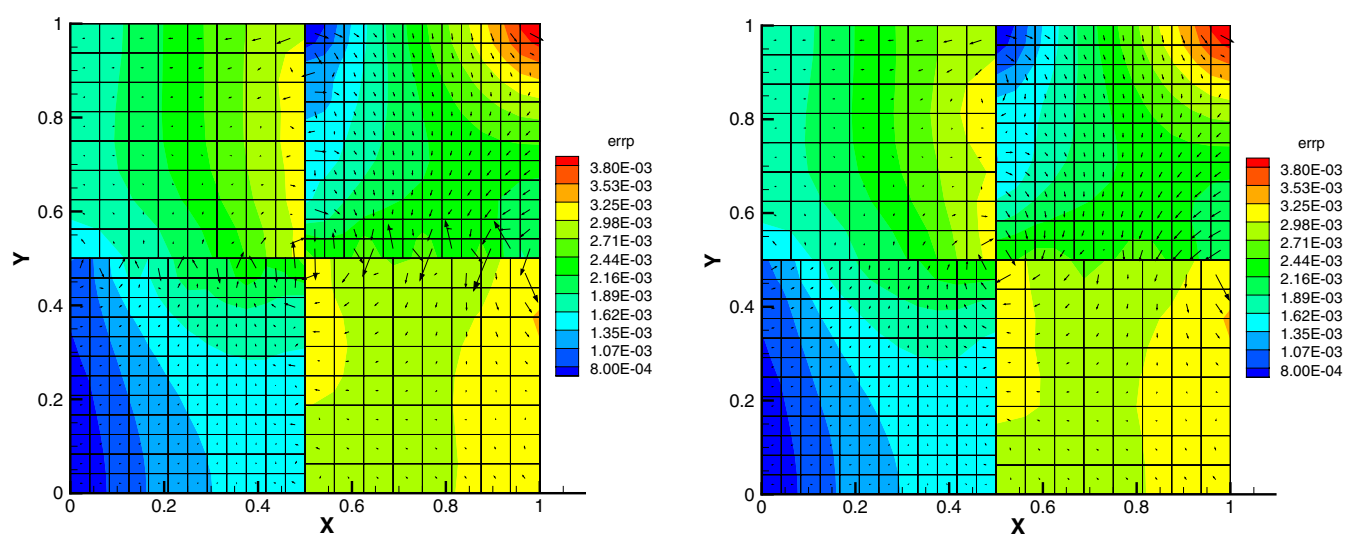

FIgURE 4. Error in the multiscale mortar MFMFE solution on nonmatching rectangular grids in Example 1: discontinuous linear (left) and discontinuous quadratic (right) mortars.

\subsection{Example 2: $h^{2}$-parallelogram mesh}

In the second example, we take the domain to be a $C^{\infty}$ map of the unit square. The map is defined as

$$
\begin{aligned}
& x=\hat{x}+0.03 \cos (3 \pi \hat{x}) \cos (3 \pi \hat{y}), \\
& y=\hat{y}-0.04 \cos (3 \pi \hat{x}) \cos (3 \pi \hat{y}) .
\end{aligned}
$$

The quadrilateral grids on the different levels are defined by mapping the nonmatching rectangular grids from Example 1. More precisely, each vertex is an image of a vertex of the rectangular grid. Due to the smoothness of the global map, all elements are $h^{2}$-parallelograms.

We choose the same analytic solution and permeability tensor as in Example 1. Convergence rates are given in Tables 6, 7. As the theory predicts, we observe first order convergence for both the pressure and velocity. We also observe second order convergence for the discrete pressure error and higher than $\mathcal{O}\left(h^{1.5}\right)$ convergence for the discrete velocity error for both linear and quadratic mortars. Again, quadratic mortars are more efficient than linear mortars. The computed solution and the corresponding error with discontinuous linear and quadratic mortars on the same nonmatching quadrilateral grids are given in Figures 5 and 6. 
TABLE 6. Example 2: discontinuous linear mortars on nonmatching grids.

\begin{tabular}{c|ccccccccc}
\hline $1 / h$ & $\left\|p-p_{h}\right\|$ & & $\left\|\mathbf{u}-\mathbf{u}_{h}\right\|$ & & $\left\|p-p_{h}\right\|$ & & $\left\|\mathbf{u}-\Pi^{R T} \mathbf{u}_{h}\right\|$ & & CGiter \\
\hline 4 & $1.96 \mathrm{E}-01$ & - & $8.56 \mathrm{E}-01$ & - & $3.11 \mathrm{E}-02$ & - & $3.53 \mathrm{E}-01$ & - & 8 \\
8 & $9.66 \mathrm{E}-02$ & 1.02 & $4.19 \mathrm{E}-01$ & 1.03 & $7.46 \mathrm{E}-03$ & 2.06 & $1.17 \mathrm{E}-01$ & 1.59 & 13 \\
16 & $4.82 \mathrm{E}-02$ & 1.00 & $2.08 \mathrm{E}-01$ & 1.01 & $1.83 \mathrm{E}-03$ & 2.03 & $3.49 \mathrm{E}-02$ & 1.75 & 19 \\
32 & $2.41 \mathrm{E}-02$ & 1.00 & $1.03 \mathrm{E}-01$ & 1.01 & $4.54 \mathrm{E}-04$ & 2.01 & $9.55 \mathrm{E}-03$ & 1.87 & 28 \\
64 & $1.20 \mathrm{E}-02$ & 1.01 & $5.13 \mathrm{E}-02$ & 1.01 & $1.13 \mathrm{E}-04$ & 2.01 & $2.60 \mathrm{E}-03$ & 1.88 & 40 \\
128 & $6.02 \mathrm{E}-03$ & 1.00 & $2.56 \mathrm{E}-02$ & 1.00 & $2.82 \mathrm{E}-05$ & 2.00 & $7.36 \mathrm{E}-04$ & 1.83 & 57 \\
256 & $3.01 \mathrm{E}-03$ & 1.00 & $1.28 \mathrm{E}-02$ & 1.00 & $7.04 \mathrm{E}-06$ & 2.00 & $2.20 \mathrm{E}-04$ & 1.74 & 81 \\
\hline
\end{tabular}

TABLE 7. Example 2: discontinuous quadratic mortars on nonmatching grids.

\begin{tabular}{c|ccccccccc}
\hline $1 / h$ & $\left\|p-p_{h}\right\|$ & & $\left\|\mathbf{u}-\mathbf{u}_{h}\right\|$ & & $\left\|p-p_{h}\right\|$ & & $\left\|\mathbf{u}-\Pi^{R T} \mathbf{u}_{h}\right\|$ & & CGiter \\
\hline 4 & $1.96 \mathrm{E}-01$ & - & $8.53 \mathrm{E}-01$ & - & $3.16 \mathrm{E}-02$ & - & $3.52 \mathrm{E}-01$ & - & 11 \\
16 & $4.82 \mathrm{E}-02$ & 1.01 & $2.07 \mathrm{E}-01$ & 1.02 & $1.84 \mathrm{E}-03$ & 2.05 & $3.32 \mathrm{E}-02$ & 1.70 & 17 \\
64 & $1.20 \mathrm{E}-02$ & 1.00 & $5.12 \mathrm{E}-02$ & 1.01 & $1.13 \mathrm{E}-04$ & 2.01 & $2.25 \mathrm{E}-03$ & 1.94 & 32 \\
256 & $3.01 \mathrm{E}-03$ & 1.00 & $1.28 \mathrm{E}-02$ & 1.00 & $7.05 \mathrm{E}-06$ & 2.00 & $1.52 \mathrm{E}-04$ & 1.94 & 58 \\
\hline
\end{tabular}
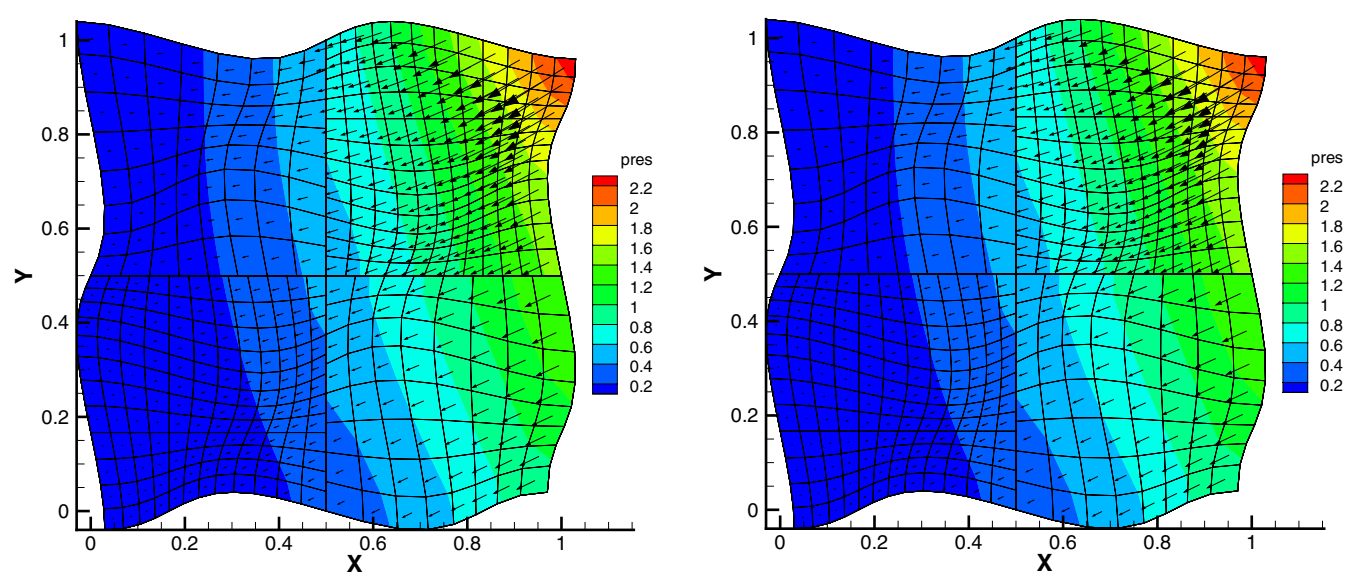

FiguRE 5. Multiscale mortar MFMFE solution on nonmatching $h^{2}$-parallelograms in Example 2: discontinuous linear (left) and discontinuous quadratic (right) mortars.

\subsection{Example 3: cuboid mesh}

In the third example, we consider cuboid grids on the unit cube with given analytic solution

$$
p(x, y, z)=x+y+z-1.5,
$$

and a full tensor coefficient

$$
K=\left(\begin{array}{ccc}
x^{2}+y^{2}+1 & 0 & 0 \\
0 & z^{2}+1 & \sin (x y) \\
0 & \sin (x y) & x^{2} y^{2}+1
\end{array}\right) .
$$

Convergence rates are reported in Tables 8 and 9. Again, these results confirm the theoretical results. Note that the discrete velocity error $\left\|\mathbf{u}-\Pi^{R T} \mathbf{u}_{h}\right\|$ has second order of convergence for both linear and quadratic mortars on matching grids, even though the theory predicts $\mathcal{O}\left(h^{1.5}\right)$ for linear mortars and $\mathcal{O}\left(h^{1.25}\right)$ for quadratic mortars. The computed solution and its error for the case of discontinuous quadratic mortars are shown in Figure 7. 
TABLE 8. Example 3: discontinuous linear mortars on matching grids.

\begin{tabular}{c|ccccccccc}
\hline $1 / h$ & $\left\|p-p_{h}\right\|$ & & $\left\|\mathbf{u}-\mathbf{u}_{h}\right\|$ & & $\left\|p-p_{h}\right\|$ & & $\left\|\mathbf{u}-\Pi^{R T} \mathbf{u}_{h}\right\|$ & & CGiter \\
\hline 4 & $2.17 \mathrm{E}-01$ & - & $1.55 \mathrm{E}-01$ & - & $9.87 \mathrm{E}-03$ & - & $3.73 \mathrm{E}-03$ & - & 14 \\
8 & $1.08 \mathrm{E}-01$ & 1.01 & $7.76 \mathrm{E}-02$ & 1.00 & $2.47 \mathrm{E}-03$ & 2.00 & $1.03 \mathrm{E}-03$ & 1.86 & 20 \\
16 & $5.41 \mathrm{E}-02$ & 1.00 & $3.88 \mathrm{E}-02$ & 1.00 & $6.17 \mathrm{E}-04$ & 2.00 & $2.60 \mathrm{E}-04$ & 1.99 & 28 \\
32 & $2.71 \mathrm{E}-02$ & 1.00 & $1.94 \mathrm{E}-02$ & 1.00 & $1.54 \mathrm{E}-04$ & 2.00 & $6.50 \mathrm{E}-05$ & 2.00 & 39 \\
64 & $1.35 \mathrm{E}-02$ & 1.01 & $9.68 \mathrm{E}-03$ & 1.00 & $3.85 \mathrm{E}-05$ & 2.00 & $1.66 \mathrm{E}-05$ & 1.97 & 56 \\
\hline
\end{tabular}

TABLE 9. Example 3: discontinuous quadratic mortars on matching grids.

\begin{tabular}{c|ccccccccc}
\hline $1 / h$ & $\left\|p-p_{h}\right\|$ & & $\left\|\mathbf{u}-\mathbf{u}_{h}\right\|$ & & $\left\|p-p_{h}\right\|$ & & $\left\|\mathbf{u}-\Pi^{R T} \mathbf{u}_{h}\right\|$ & & CGiter \\
\hline 4 & $2.17 \mathrm{E}-01$ & - & $1.55 \mathrm{E}-01$ & - & $9.87 \mathrm{E}-03$ & - & $3.73 \mathrm{E}-03$ & - & 14 \\
16 & $5.41 \mathrm{E}-02$ & 1.00 & $3.88 \mathrm{E}-02$ & 1.00 & $6.17 \mathrm{E}-04$ & 2.00 & $2.61 \mathrm{E}-04$ & 1.92 & 27 \\
64 & $1.35 \mathrm{E}-02$ & 1.00 & $9.68 \mathrm{E}-03$ & 1.00 & $3.85 \mathrm{E}-05$ & 2.00 & $1.67 \mathrm{E}-05$ & 1.98 & 53 \\
\hline
\end{tabular}
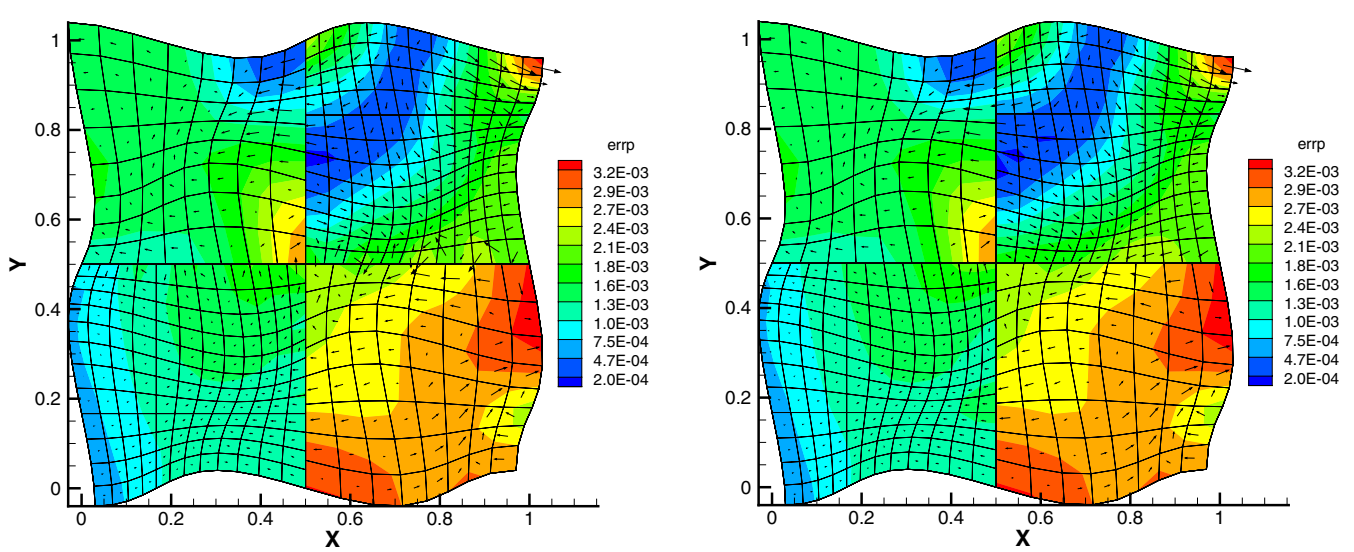

FIgURE 6. Error in the multiscale mortar MFMFE solution on nonmatching $h^{2}$-parallelograms in Example 2: discontinuous linear (left) and discontinuous quadratic (right) mortars.

\subsection{Example 4: regular $h^{2}$-parallelepiped mesh}

In this example, we take the domain to be a $C^{\infty}$ map of the unit cube. The map is defined as

$$
\begin{aligned}
& x=\hat{x}+0.03 \cos (3 \pi \hat{x}) \cos (3 \pi \hat{y}) \cos (3 \pi \hat{z}), \\
& y=\hat{y}-0.04 \cos (3 \pi \hat{x}) \cos (3 \pi \hat{y}) \cos (3 \pi \hat{z}), \\
& z=\hat{z}+0.05 \cos (3 \pi \hat{x}) \cos (3 \pi \hat{y}) \cos (3 \pi \hat{z}) .
\end{aligned}
$$

The computational grids are defined by mapping the cuboid grids considered in Example 3. Each vertex of a hexahedral element is obtained by mapping a vertex in the cuboid grid. The element trilinear mapping determines the shape of each hexahedron. The smoothness of the global mapping implies that each hexahedron is a regular $h^{2}$-parallelepiped.

We choose the same analytic solution and permeability tensor as in Example 3. Tables 10 and 11 show the convergence rates for discontinuous linear and quadratic mortars on matching grids. Again, these results confirm the theory and show higher than the theoretical order of convergence for the discrete pressure and velocity errors. The computed solution and the corresponding error for the case of discontinuous quadratic mortars are shown in Figure 8. 
TABLE 10. Example 4: discontinuous linear mortars on matching grids.

\begin{tabular}{c|ccccccccc}
\hline $1 / h$ & $\left\|p-p_{h}\right\|$ & & $\left\|\mathbf{u}-\mathbf{u}_{h}\right\|$ & & $\left\|p-p_{h}\right\|$ & & $\left\|\mathbf{u}-\Pi^{R T} \mathbf{u}_{h}\right\|$ & & CGiter \\
\hline 4 & $2.18 \mathrm{E}-01$ & - & $2.82 \mathrm{E}-01$ & - & $1.39 \mathrm{E}-02$ & - & $7.48 \mathrm{E}-02$ & - & 14 \\
8 & $1.10 \mathrm{E}-01$ & 0.99 & $1.66 \mathrm{E}-01$ & 0.76 & $5.07 \mathrm{E}-03$ & 1.46 & $5.15 \mathrm{E}-02$ & 0.54 & 20 \\
16 & $5.49 \mathrm{E}-02$ & 1.00 & $8.96 \mathrm{E}-02$ & 0.89 & $1.86 \mathrm{E}-03$ & 1.45 & $2.09 \mathrm{E}-02$ & 1.30 & 28 \\
32 & $2.75 \mathrm{E}-02$ & 1.00 & $4.51 \mathrm{E}-02$ & 0.99 & $5.24 \mathrm{E}-04$ & 1.83 & $5.93 \mathrm{E}-03$ & 1.82 & 40 \\
64 & $1.37 \mathrm{E}-02$ & 1.01 & $2.23 \mathrm{E}-02$ & 1.02 & $1.35 \mathrm{E}-04$ & 1.96 & $1.52 \mathrm{E}-03$ & 1.96 & 57 \\
\hline
\end{tabular}

TABLE 11. Example 4: discontinuous quadratic mortars on matching grids.

\begin{tabular}{c|ccccccccc}
\hline $1 / h$ & $\left\|p-p_{h}\right\|$ & & $\left\|\mathbf{u}-\mathbf{u}_{h}\right\|$ & & $\left\|p-p_{h}\right\|$ & & $\left\|\mathbf{u}-\Pi^{R T} \mathbf{u}_{h}\right\|$ & & CGiter \\
\hline 4 & $2.18 \mathrm{E}-01$ & - & $2.82 \mathrm{E}-01$ & - & $1.39 \mathrm{E}-02$ & - & $7.48 \mathrm{E}-02$ & - & 14 \\
16 & $5.49 \mathrm{E}-02$ & 0.99 & $8.96 \mathrm{E}-02$ & 0.83 & $1.86 \mathrm{E}-03$ & 1.45 & $2.09 \mathrm{E}-02$ & 0.92 & 27 \\
64 & $1.37 \mathrm{E}-02$ & 1.00 & $2.24 \mathrm{E}-02$ & 1.00 & $1.35 \mathrm{E}-04$ & 1.89 & $1.53 \mathrm{E}-03$ & 1.89 & 54 \\
\hline
\end{tabular}
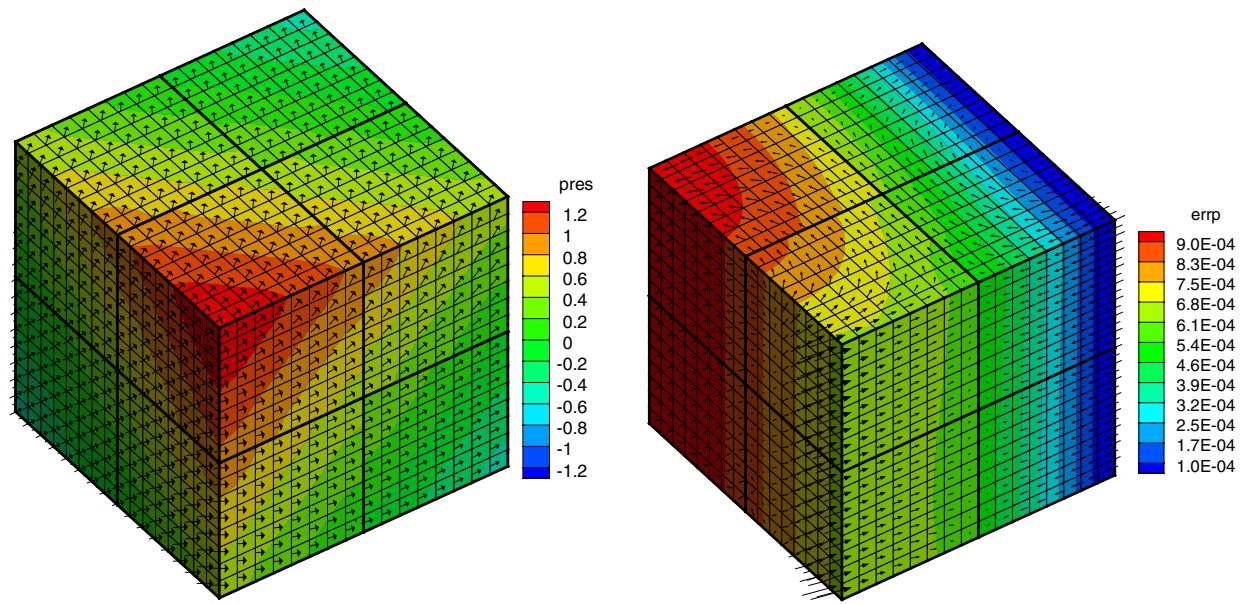

Figure 7. Multiscale mortar MFMFE solution (left) and error (right) for Example 3: discontinuous quadratic mortars on matching grids.

\subsection{Example 5: heterogeneous permeability}

We use a heterogeneous permeability from the Society of Petroleum Engineers (SPE) Comparative Solution Project ${ }^{4}$. The computation domain is $\Omega=(0,120)^{2}$ with a fixed rectangular $120 \times 120$ grid. The left and right boundary conditions are $p=0$ and $p=1$. No flow is specified on the top and bottom boundaries. The permeability field is shown in Figure 9.

A comparison between the fine scale solution and the multiscale solution with $6 \times 6$ subdomains and a single quadratic mortar per interface is shown in Figure 9. We observe a very good match between the two solutions. At the same time, the multiscale solution is significantly less expensive than the fine scale solution. This can be observed in Table 12, where we compare the cost for computing a multiscale solution with several different mortar grids with the cost for the fine scale solution. We present both the cost for the original implementation and the cost for multiscale flux basis implementation. Since the dominant cost is in solving subdomain problems, we report the number of solves per subdomain. Recall that in the original implementation, one set of subdomain

\footnotetext{
${ }^{4}$ http://www.spe.org/web/csp/
} 
TABLE 12. Example 5: $6 \times 6$ subdomains

\begin{tabular}{l|ccc}
\hline Mortar type & $\begin{array}{c}\text { \# of elements } \\
\text { per interface }\end{array}$ & $\begin{array}{c}\text { Original implementation } \\
\text { Solves }\end{array}$ & $\begin{array}{c}\text { MS flux basis } \\
\text { Solves per subdomain }\end{array}$ \\
\hline Discontinuous linear & 1 & 112 & 11 \\
Continuous quadratic & 1 & 132 & 15 \\
Discontinuous quadratic & 1 & 170 & 15 \\
Continuous linear & 2 & 108 & 15 \\
Discontinuous linear & 2 & 173 & 19 \\
Piecewise constant & 20 & 539 & 83 \\
(fine scale solution) & & & \\
\hline
\end{tabular}
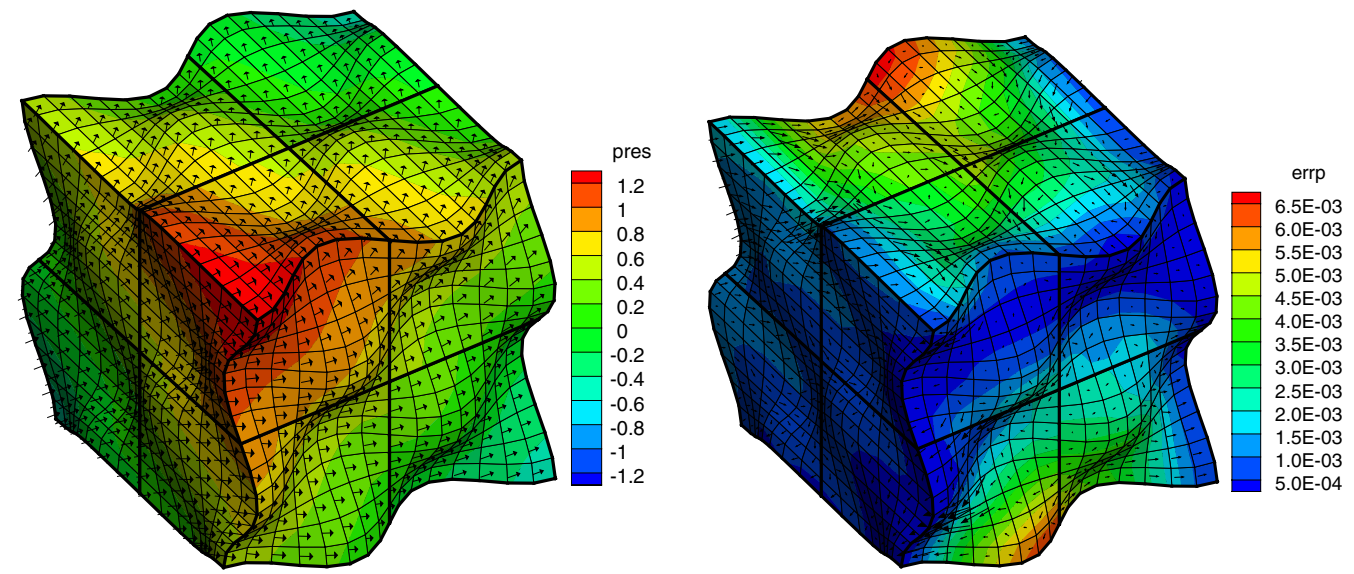

Figure 8. Multiscale mortar MFMFE solution (left) and error (right) for Example 4: discontinuous quadratic mortars on matching grids.
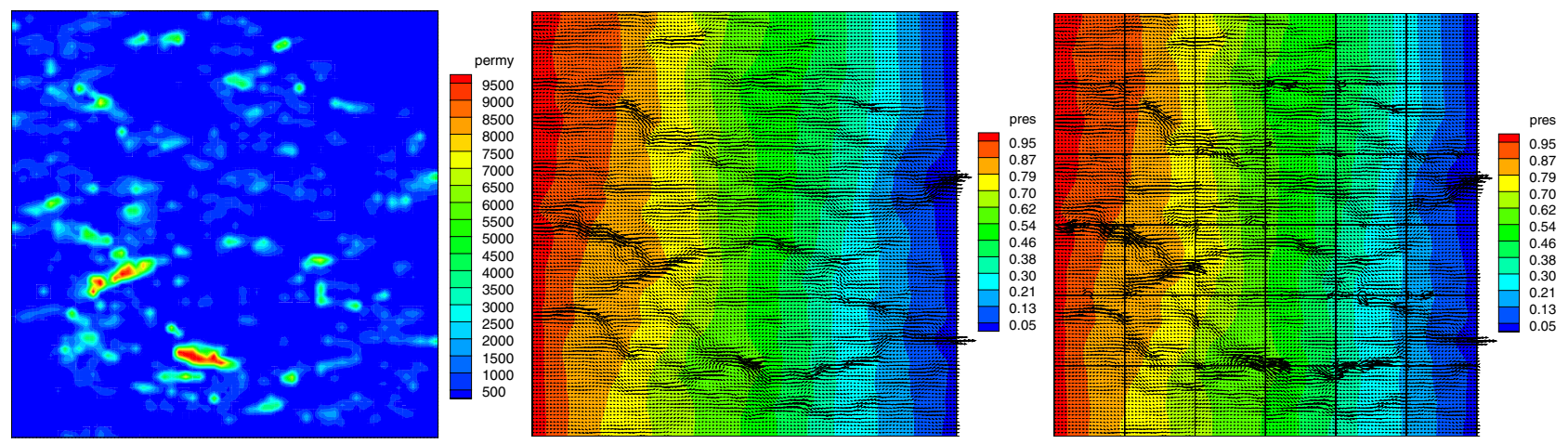

Figure 9. Permeability (left), fine scale solution (middle), and multiscale solution with one single quadratic mortar per interface (right). 
problems needs to be solved at each CG iteration. There are three additional solves needed, so the total number of solves per subdomain equals the number of CG iterations +3 . In the multiscale flux basis implementation, subdomain solves are only needed to compute the basis. The number of solves for each subdomain equals number of mortar degrees of freedom +3 . We can make two conclusions. First, the fine scale solution (reported in the last line - piecewise constant mortars with 20 elements per interface) requires a significantly larger number of subdomain solves than the multiscale solution. Second, for these test cases the multiscale flux basis implementation is computationally more efficient than the original implementation.

\section{REFERENCES}

[1] J.E. Aarnes, S. Krogstad and K.-A. Lie, A hierarchical multiscale method for two-phase flow based on mixed finite elements and nonuniform coarse grids. Multiscale Model. Simul. 5 (2006) 337-363.

[2] J.E. Aarnes, Y. Efendiev and L. Jiang, Mixed multiscale finite element methods using limited global information. Multiscale Model. Simul. 7 (2008) 655-676.

[3] I. Aavatsmark, An introduction to multipoint flux approximations for quadrilateral grids. Comput. Geosci. 6 (2002) 405-432.

[4] I. Aavatsmark, T. Barkve, $\varnothing$. Bøe and T. Mannseth, Discretization on unstructured grids for inhomogeneous, anisotropic media. I. Derivation of the methods. SIAM J. Sci. Comput. 19 (1998) 1700-1716.

[5] I. Aavastsmark, G.T. Eigestad, R.A. Klausen, M.F. Wheeler and I. Yotov, Convergence of a symmetric MPFA method on quadrilateral grids. Comput. Geosci. 11 (2007) 333-345.

[6] I. Aavatsmark, G.T. Elgestad, B.T. Mallison and J.M. Nordbotten, A compact multipoint flux approximation method with improved robustness. Numer. Methods for Partial Differential Equations 24 (2008) 1329-1360.

[7] L. Agélas, D.A. Di Pietro and J. Droniou, The G method for heterogeneous anisotropic diffusion on general meshes. Math. Model. Numer. Anal. 44 (2010) 597-625.

[8] T. Arbogast, Analysis of a two-scale, locally conservative subgrid upscaling for elliptic problems. SIAM. J. Numer. Anal. 42 (2004) 576-598.

[9] T. Arbogast, M.F. Wheeler and I. Yotov, Mixed finite elements for elliptic problems with tensor coefficients as cell-centered finite differences. SIAM J. Numer. Anal. 34 (1997) 828-852.

[10] T. Arbogast, C.N. Dawson, P.T. Keenan, M.F. Wheeler and I. Yotov, Enhanced cell-centered finite differences for elliptic equations on general geometry. SIAM J. Sci. Comput. 19 (1998) 404-425.

[11] T. Arbogast, L.C. Cowsar, M.F. Wheeler and I. Yotov, Mixed finite element methods on nonmatching multiblock grids. SIAM J. Numer. Anal. 37 (2000) 1295-1315.

[12] T. Arbogast, G. Pencheva, M.F. Wheeler and I. Yotov, A multiscale mortar mixed finite element method. Multiscale Model. Simul. 6 (2007) 319-346.

[13] D.N. Arnold and F. Brezzi, Mixed and nonconforming finite element methods: implementation, postprocessing and error estimates. RAIRO Modèl. Math. Anal. Numèr. 19 (1985) 7-32.

[14] D.N. Arnold, D. Boffi and R.S. Falk, Quadrilateral H(div) finite elements. SIAM J. Numer. Anal. 42 (2005) $2429-2451$.

[15] J. Baranger, J.F. Maitre and F. Oudin, Connection between finite volume and mixed finite element methods. RAIRO Modèl. Math. Anal. Numèr. 30 (1996) 445-465.

[16] C. Bernardi, Y. Maday and A.T. Patera. A new nonconforming approach to domain decomposition: The mortar element method, in Nonlinear Partial Differential Equations and Their Applications, edited by H. Brezis and J.L. Lions. Longman Scientific and Technical, Harlow, UK (1994).

[17] M. Berndt, K. Lipnikov, M. Shashkov, M.F. Wheeler and I. Yotov, Superconvergence of the velocity in mimetic finite difference methods on quadrilaterals. SIAM. J. Numer. Anal. 43 (2005) 1728-1749.

[18] S.C. Brenner and L.R. Scott, The Mathematical Theory of Finite Element Methods. Texts in Applied Mathematics, SpringerVerlag (2007).

[19] F. Brezzi and M. Fortin, Mixed and hybrid finite element methods. Springer-Verlag, New York (1991).

[20] F. Brezzi, J. Douglas and L.D. Marini, Two families of mixed finite elements for second order elliptic problems. Numer. Math. 47 (1985) 217-235.

[21] F. Brezzi, J. Douglas, R. Duran and M. Fortin, Mixed finite elements for second order elliptic problems in three variables. Numer. Math. 51 (1987) 237-250. 
[22] F. Brezzi, M. Fortin and L.D. Marini, Error analysis of piecewise constant pressure approximations of Darcy's law. Comput. Methods Appl. Mech. Engrg. 195 (2006) 1547-1559.

[23] Z. Cai, J.E. Jones, S.F. McCormick and T.F. Russell, Control-volume mixed finite element methods. Comput. Geosci. 1 (1997) 289-315 (1998).

[24] Z. Chen and T.Y. Hou, A mixed multiscale finite element method for elliptic problems with oscillating coefficients. Math. Comp. 72 (2003) 541-576.

[25] P.G. Ciarlet, The Finite Element Method for Elliptic Problems, Stud. Math. Appl. 4. North-Holland, Amsterdam (1978); reprinted, SIAM, Philadelphia (2002).

[26] R. Duran, Superconvergence for rectangular mixed finite elements. Numer. Math. 58 (1990) $287-298$.

[27] M.G. Edwards, Unstructured, control-volume distributed, full-tensor finite-volume schemes with flow based grids. Comput. Geosci. 6 (2002) 433-452.

[28] M.G. Edwards and C.F. Rogers, Finite volume discretization with imposed flux continuity for the general tensor pressure equation. Comput. Geosci. 2 (1998) 259-290 (1999).

[29] R.E. Ewing, M.M. Liu and J. Wang, Superconvergence of mixed finite element approximations over quadrilaterals. SIAM. J. Numer. Anal. 36 (1999) 772-787.

[30] R. Eymard, T. Gallouet and R. Herbin, Finite volume methods. in Handbook of Numerical Analysis. North-Holland, Amsterdam (2000) 713-1020.

[31] G.P. Galdi, An introduction to the mathematical theory of the Navier-Stokes equations I. Linearized steady problems, SpringerVerlag, New York (1994)

[32] B. Ganis and I. Yotov, Implementation of a mortar mixed finite element using a multiscale flux basis. Comput. Methods Appl. Mech. Engrg. 198 (2009) 3989-3998.

[33] V. Girault and P.A. Raviart, Finite Element Methods for Navier-Stokes Equations, Theory and Algorithms. Springer-Verlag (1986).

[34] R. Glowinski and M.F. Wheeler, Domain decomposition and mixed finite element methods for elliptic problems, in First International Symposium on Domain Decomposition Methods for Partial Differential Equations, edited by R. Glowinski, G.H. Golub, G.A. Meurant and J. Periaux. SIAM, Philadelphia (1988) 144-172.

[35] P. Grisvard, Elliptic Problems in Nonsmooth Domains. Pitman, Boston, MA (1995).

[36] T.Y. Hou and X.H. Wu, A multiscale finite element method for elliptic problems in composite materials and porous media. J. Comput. Phys. 134 (1997) 169-189.

[37] T.J.R. Hughes, G.R. Feijoo, L. Mazzei and J.-B. Quincy, The variational multiscale method-a paradim for computational mechanics. Comput. Methods Appl. Mech. Engrg. 166 (1998) 3-24.

[38] J. Hyman, M. Shashkov and S. Steinberg, The numerical solution of diffusion problem in strongly heterogeneous non-isotropic materials. J. Comput. Phys. 132 (1997) 130-148.

[39] R. Ingram, M.F. Wheeler and I. Yotov, A multipoint flux mixed finite element method on hexahedra. SIAM J. Numer. Anal. 48 (2010) 1281-1312.

[40] P. Jenny, S.H. Lee and H.A. Tchelepi, Multi-scale finite-volume method for elliptic problems in subsurface flow simulation. J. Comput. Phys. 187 (2003) 47-67.

[41] R.A. Klausen and R. Winther, Robust convergence of multi point flux approximation on rough grids. Numer. Math. 104 (2006) $317-337$.

[42] R.A. Klausen and R. Winther, Convergence of multipoint flux approximations on quadrilateral grids. Numer. Methods Partial Differential Equations 22 (2006) 1438-1454.

[43] J.L. Lions and E. Magenes, Non-homogeneous Boundary Value Problems and Applications. Springer-Verlag, Berlin, Heidelberg, New York (1972).

[44] K. Lipnikov, M. Shashkov and I. Yotov, Local flux mimetic finite difference methods. Numer. Math. 112 (2009) $115-152$.

[45] T.P. Mathew, Domain Decomposition and Iterative Methods for Mixed Finite Element Discretizations of Elliptic Problems. Tech. Report 463, Courant Institute of Mathematical Sciences, New York University, New York (1989).

[46] J.C. Nedelec, Mixed finite elements in $R^{3}$. Numer. Math. 35 (1980) 315-341.

[47] G. Pencheva and I. Yotov, Balancing domain decomposition for mortar mixed finite element methods on non-matching grids. Numer. Linear Algebra Appl. 10 (2003) 159-180.

[48] P.A. Raviart and J. Thomas, A mixed finite element method for 2-nd order elliptic problems, in Mathematical aspects of the Finite Elements Method, Lect. Notes Math. 606 (1977) 292-315.

[49] J.E. Roberts and J.-M. Thomas, Mixed and hybrid methods, in Handbook of Numerical Analysis II, edited by P.G. Ciarlet and J.L. Lions. Elsevier Science Publishers B.V. (1991) 523-639.

[50] T.F. Russell and M.F. Wheeler, Finite element and finite difference methods for continuous flows in porous media, in The Mathematics of Reservoir Simulation, edited by R.E. Ewing. SIAM, Philadelphia (1983) 35-106.

[51] R. Scott and S. Zhang, Finite element interpolation of nonsmooth functions satisfying boundary conditions. Math. Comp. 54 (1990) 483-493. 
[52] J.M. Thomas, These de Doctorat d'etat, Sur l'analyse numérique des méthodes d'éléments finis hybrides et mixtes. Ph.D. thesis, à l'Université Pierre et Marie Curie (1977).

[53] M. Vohralík, Equivalence between lowest-order mixed finite element and multi-point finite volume methods on simplicial meshes. ESAIM:M2AN 40 (2006) 367-391.

[54] J. Wang and T.P. Mathew, Mixed finite element method over quadrilaterals, in Conference on Advances in Numerical Methods and Applications, edited by I.T. Dimov, B. Sendov and P. Vassilevski. World Scientific, River Edge, NJ (1994) $351-375$.

[55] A. Weiser and M.F. Wheeler, On convergence of block-centered finite differences for elliptic problems. SIAM J. Numer. Anal. 25 (1988) 351-375.

[56] M.F. Wheeler and I. Yotov, A multipoint flux mixed finite element method. SIAM. J. Numer. Anal. 44 (2006) $2082-2106$.

[57] M.F. Wheeler, G. Xue and I. Yotov, A multipoint flux mixed finite element method on distorted quadrilaterals and hexahedra. Accepted by Numer. Math. (2011).

[58] A. Younès, P. Ackerer and G. Chavent, From mixed finite elements to finite volumes for elliptic PDEs in two and three dimensions. Internat. J. Numer. Methods Engrg. 59 (2004) 365-388. 